\title{
Electrical Control of Droplet formation in Microfluidic Devices
}

Tool for active droplet generation in droplet microfluidics

\author{
Dissertation \\ for the award of the degree \\ "Doctor rerum natualium" \\ Division of Mathematics and Natural Sciences \\ of the Georg-August-Universität Göttingen
}

within the doctoral program (PBCS)

of the Georg-August University School of Science (GAUSS)

submitted by

Say Hwa Tan

From Singapore

Göttingen, 2014 


\section{Thesis Committee}

Prof. Dr. Jean-Christophe Baret (Reviewer)

Independent Group Droplets, Membranes and Interfaces (DMI), Max Planck Institute for Dynamics and Self-Organisation

Prof. Dr. Joerg Enderlein (Reviewer)

Third Institute of Physics, Biophysics and Complex System, Georg-August-Universität Göttingen

Prof. Dr. Stephan Herminghaus (Co-reviewer)

Dept. of Dynamics of Complex Fluids, Max Planck Institute for Dynamics and Self-Organisation

\section{Members of the examination board}

Prof. Dr. Sarah Köster

Institute for X-Ray Physics,

Georg-August-Universität Göttingen

Prof. Dr. Tim Salditt

Institute for X-Ray Physics,

Georg-August-Universität Göttingen

Dr. Thomas P. Burg

Max Planck Research Group Micro- and Nanotechnology,

Max Planck Institute for BioPhysical Chemistry

Date of oral examination: 
The work described in this doctoral thesis was carried out under the guidance and supervision of Prof. Dr. Jean-Christophe Baret in the group of Droplets, Membranes and Interfaces (DMI) of the Max Planck Institute for Dynamics and Self-Organisation, Göttingen, between September 2010 and Jun 2014.

It was supported by the Max Planck Society (International Max Planck Research School- Physics of Biological and Complex Systems).

It is declared that the presented thesis has been written independently and with no other sources and aids than quoted.

Göttingen, June 2014 


\section{I don't know anything, but I know that everything is interesting if you go into it deeply enough.}

Richard P.Feynman, The Pleasure of finding things out. 


\section{Abstract}

This thesis presents both experimental investigations and demonstration on the use of an AC voltage induced electric field to actively control the size or frequencies of droplet generated in a microfluidic flow focusing device. In the first part, we demonstrate our patented technology where the concept is realized as a new method of controlling the droplet sizes or frequencies. We show that the concept can be applied for both the case of with and without the orifice in the microfluidic flow focusing geometry.

Next, we systematically investigate the influences of various parameters such as frequencies of sinusoidal signal, conductivities of the dispersed phase fluid, different electrode configurations, ionic properties of the dispersed phase fluid and different volumetric flow rates. We present different electrical circuits to model the different electrode configurations and show that the voltage at the tip of the dispersed phase fluid is the underlying cause for the experimental observations seen in the different conditions. Using the voltage divider rule, the voltage at the tip of the dispersed phase fluid is estimated using the ratio of the frequency $(f)$ of the signal to the conductivity $(\kappa)$ of the dispersed phase fluid. In the case of electrode in configuration A, we observed that the system behaves akin to a high pass filter around the value of about $\mathrm{f} / \mathrm{k}=4 \times 10^{5} \mathrm{~m} / \mathrm{F}$ when the experimental data are rescaled. Phase diagrams illustrating the effect of both the frequencies and conductivities of the different electrode configurations are also presented. In electrode configuration A and C, we observed a transition from the unstable jetting to the dripping mode at a similar value of about $\mathrm{f} / \mathrm{K}=5 \times 10^{5} \mathrm{~m} / \mathrm{F}$. This result agrees to our hypothesis where the voltage at the tip of the dispersed phase fluid decreases significantly behaving akin to a high pass filter below the stated ratio of $\mathrm{f} / \mathrm{K}$.

We also present an electrohydrodynamic model to account for both hydrodynamic and Maxwell stresses using an effective capillary number $C a_{\text {eff. }}$. This non-dimensional number takes into account a capillary number $C a$ and an electrical bond number $B_{e}$. Using the model, the diameters of the droplets can also be estimated by different power laws when formed in either the dripping or jetting regime. However, this model does not explain the transition between the droplet formation regimes. 
In the dynamical studies, we present the concept of using this $A C$ voltage induced electric field to play musical sound tracks. A standard epifluorescence optical setup is used to detect the frequencies of the generated droplets. The frequencies of the generated droplets are then modulated by changing the applied $A C$ voltage to play different musical notes. We examine the frequency range of the system and show that the range allows access to play all the different musical notes within one octave. We also examine the response time of the system using an amplitude modulation signal at different voltages and frequencies. Experimental results show that the system is capable of modulating the frequencies of the generated droplets in the order of milli-seconds which is suitable for playing musical sound tracks. Lastly, we use this high speed of control in the droplet formation frequencies as a microfluidic jukebox. Different musical song tracks namely Ode to Joy and The flight of the bumblebee are played by rapidly changing the droplet generation frequencies using different $A C$ voltages. 


\section{Contents}

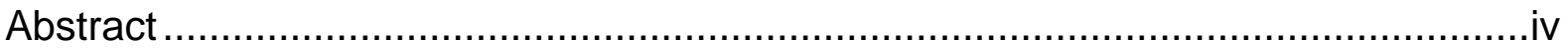

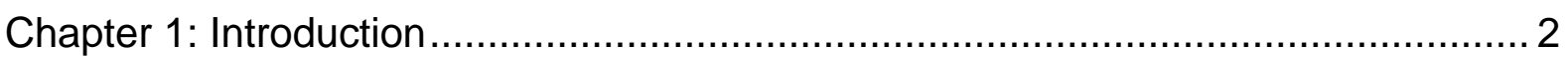

1.1 "Why are people interested in active droplet generation?" .......................... 2

1.2 Droplet generation and manipulation ............................................... 3

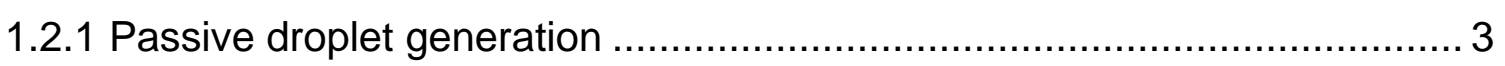

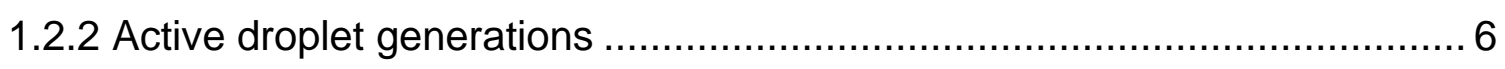

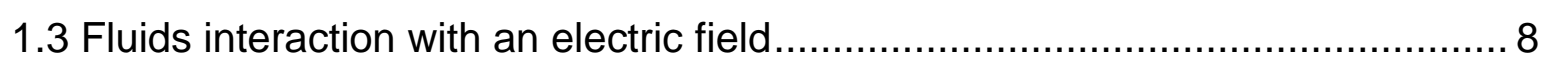

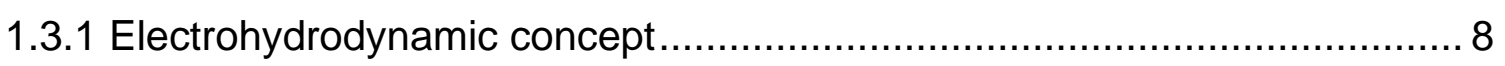

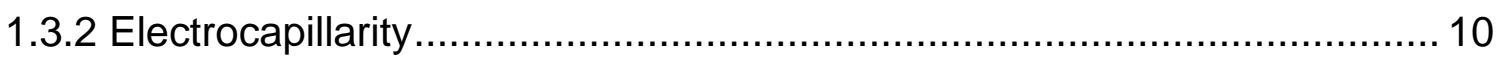

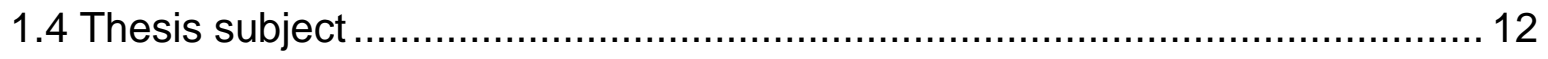

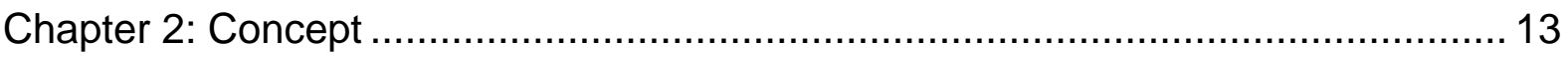

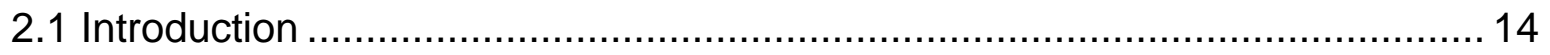

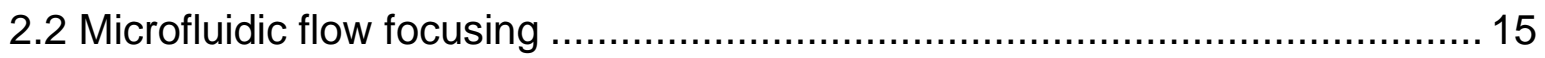

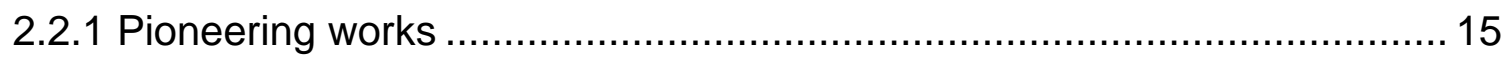

2.2.2 Three dimensional (3D) microfluidic devices ................................. 16

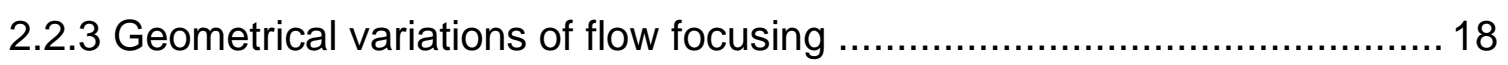

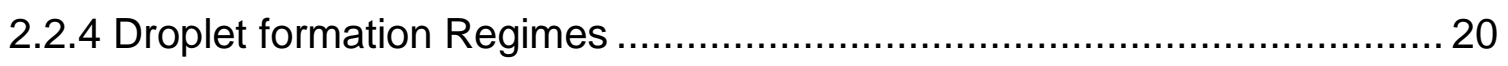

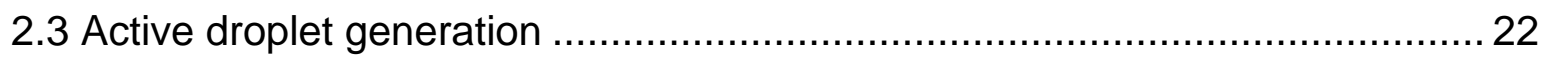

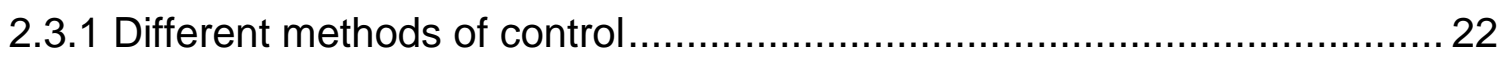

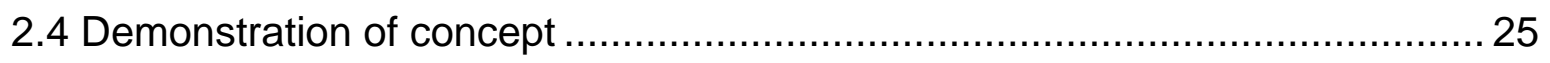

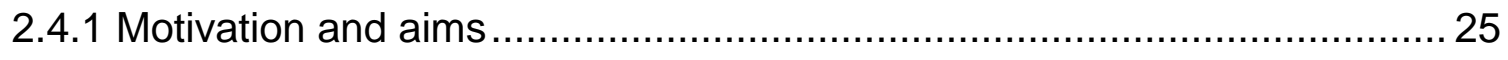

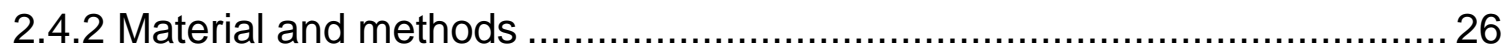

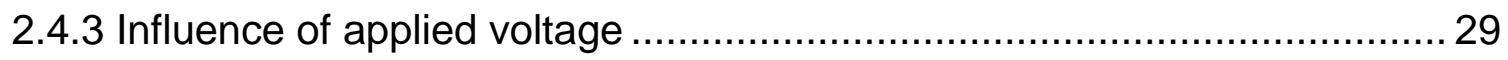

2.4.4 Influence of applied frequency ..................................................... 30 
2.5 Discussion

2.5.1 Geometrical alteration .................................................................. 32

2.5.2 Other factors to be considered.................................................. 34

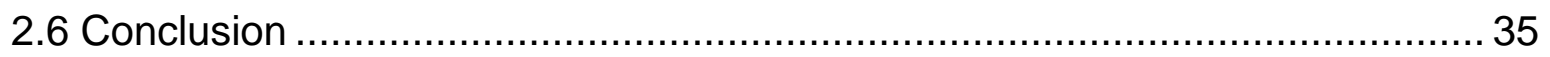

Chapter 3: Microfluidic flow-focusing in ac electric fields................................. 38

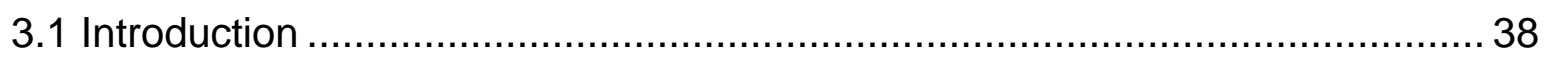

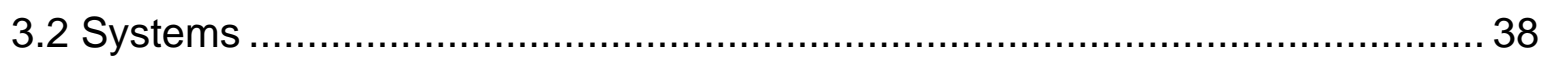

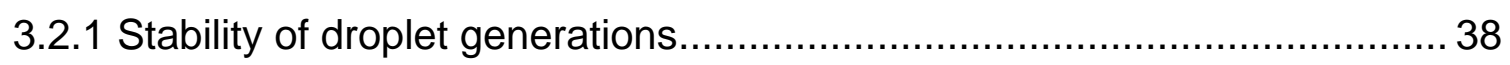

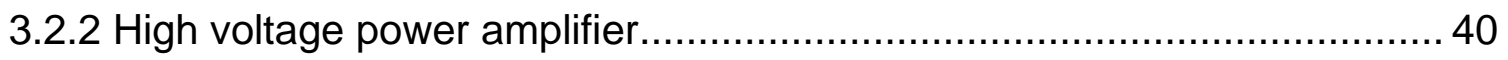

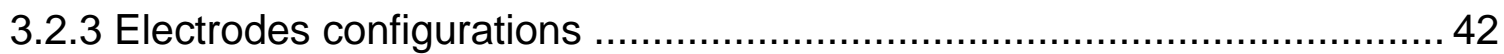

3.2.4 Interfacial tension measurements ................................................... 43

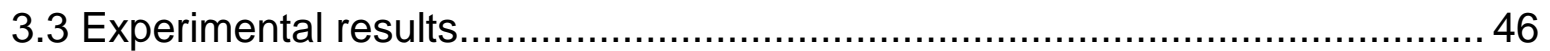

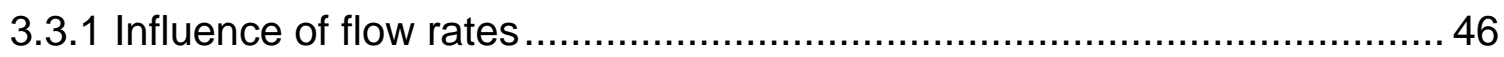

3.3.2 Influence of the conductivities of the dispersed phase fluid ....................50

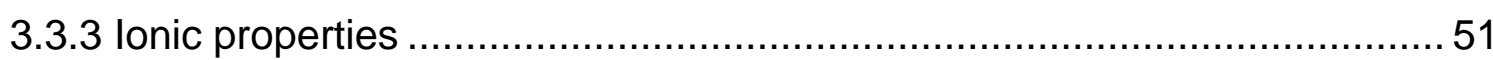

3.3.4 Coupling of frequencies and conductivities................................... 52

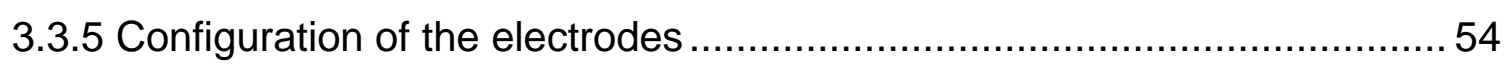

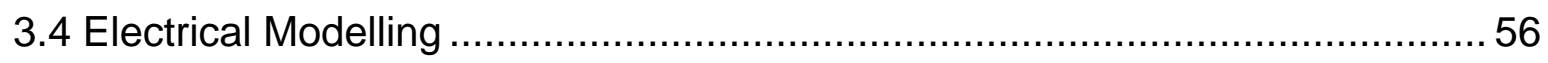

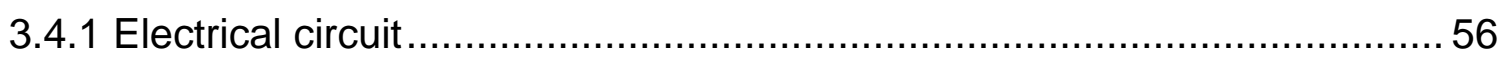

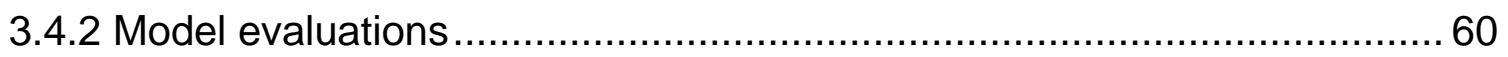

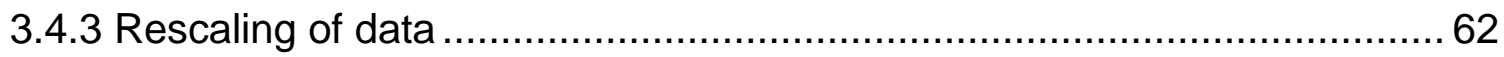

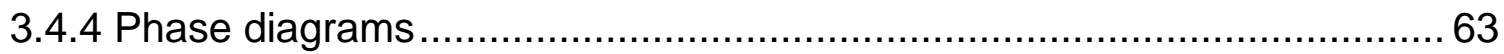

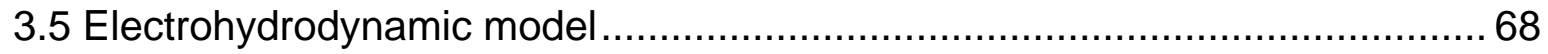

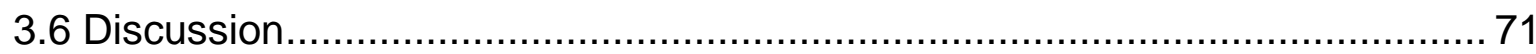

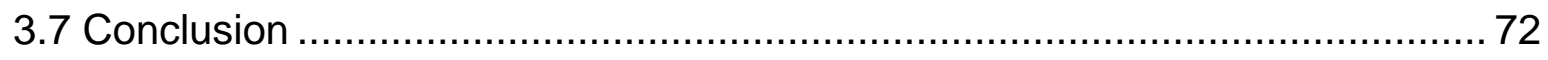

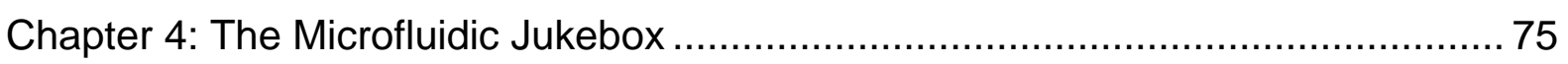

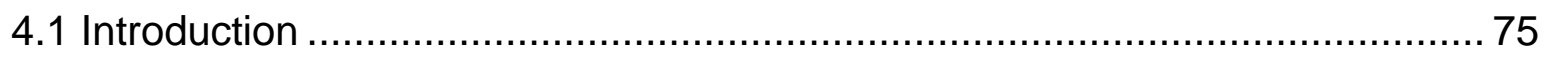


4.2 System

4.2.1 Electrical Control of droplet production ................................................ 75

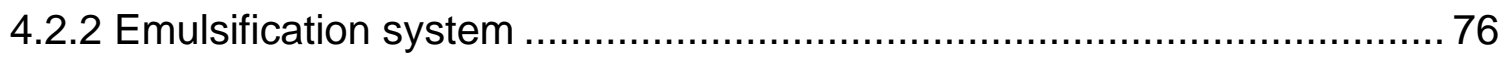

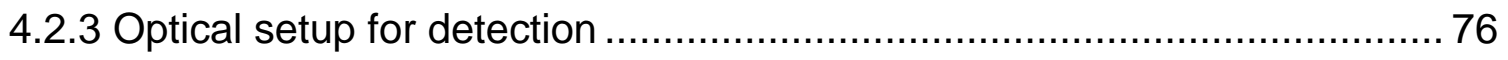

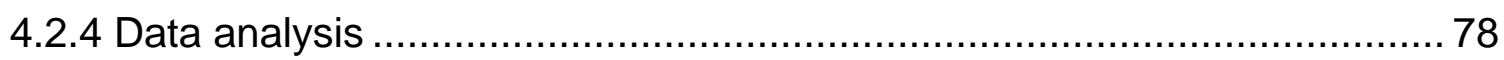

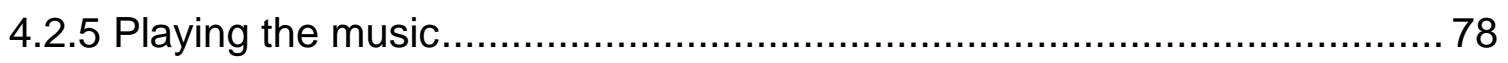

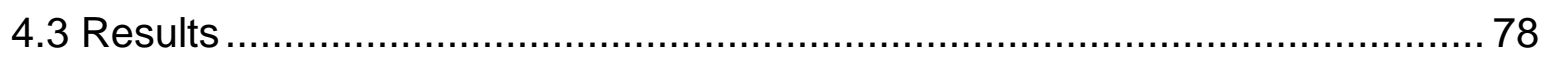

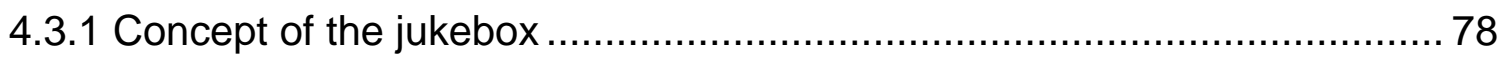

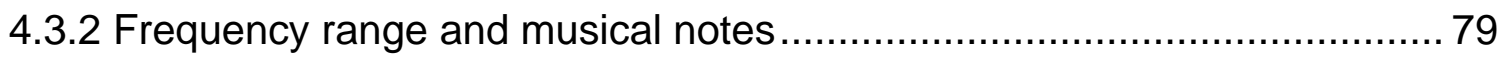

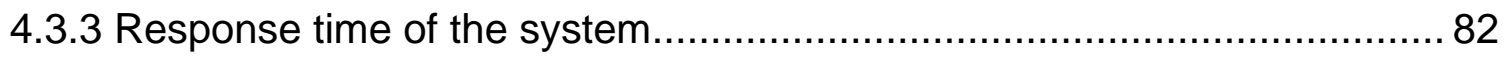

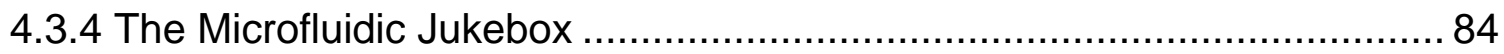

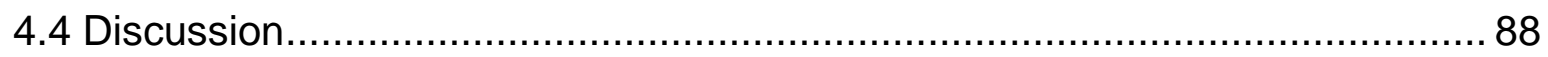

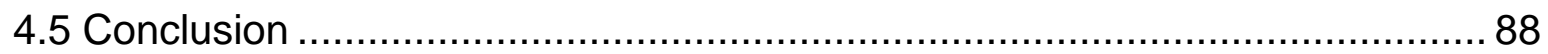

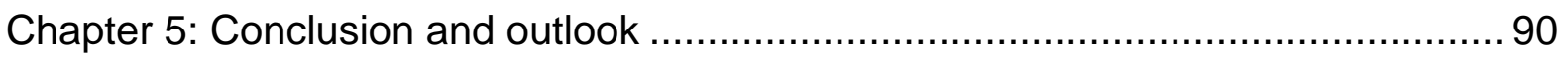

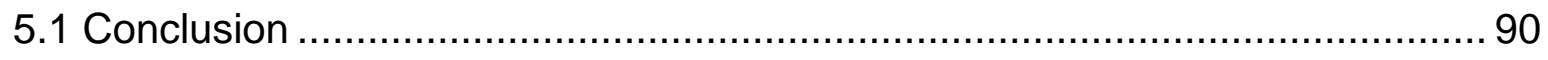

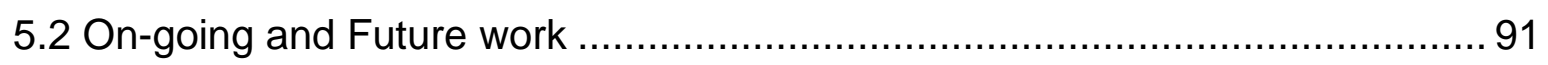

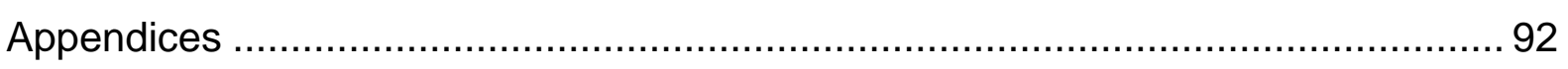

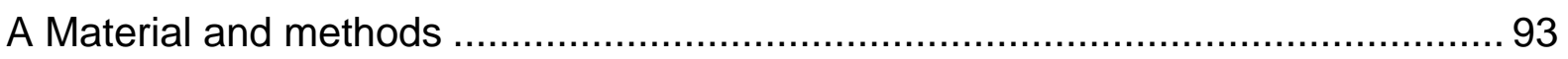

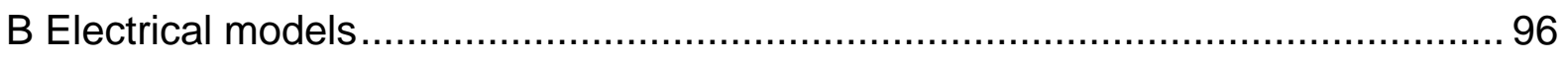

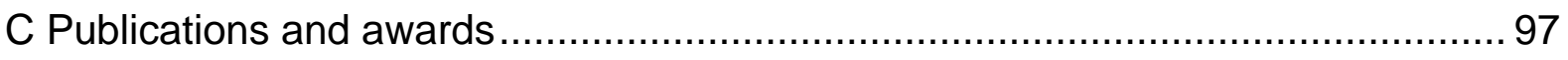

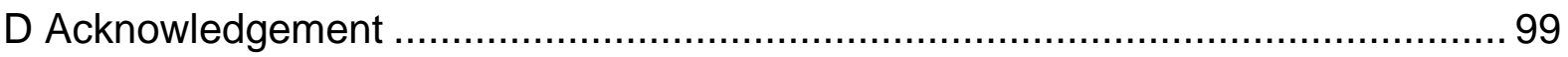

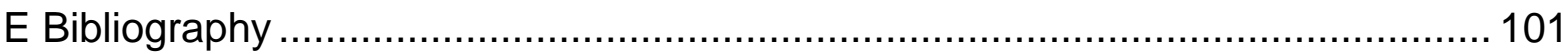

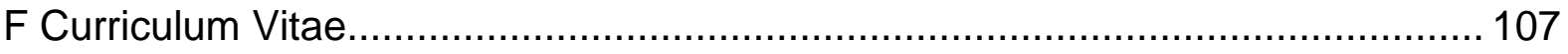




\section{Part 1}

\section{Chapter 1: Introduction}

Content

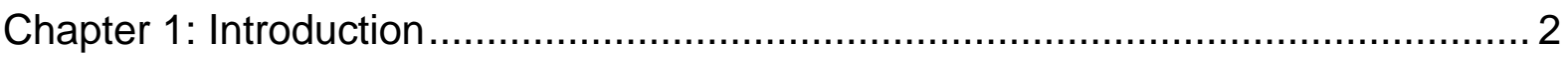

1.1 "Why are people interested in active droplet generation?" .............................. 2

1.2 Droplet generation and manipulation ..................................................... 3

1.2.1 Passive droplet generation ................................................................ 3

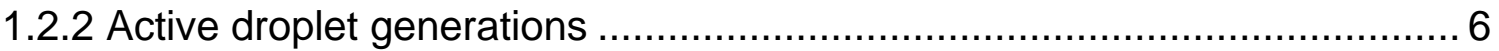

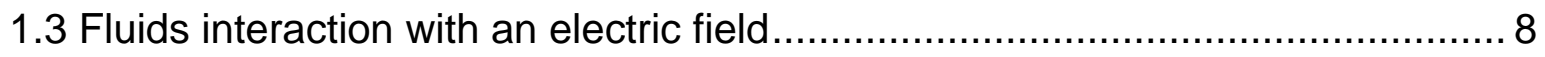

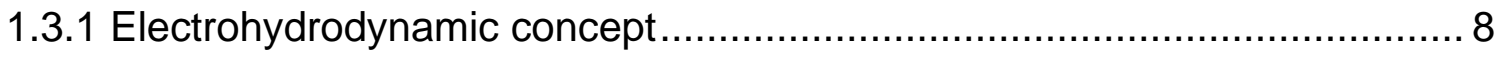

1.3.2 Electrocapillarity ....................................................................... 10

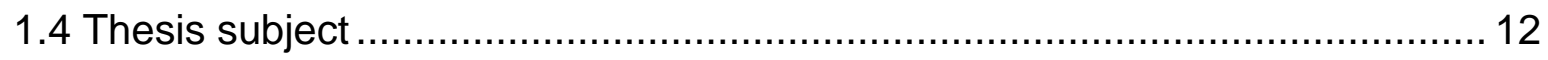




\section{Chapter 1: Introduction}

\section{1 "Why are people interested in active droplet generation?"}

This question has been pondering over my mind since the start my PhD tenure. Are people really interested in this? What does this bring to the field? I found that the answers to the question are implicit and multi-fold.

The first part of the answer to the question is that droplet microfluidics is a field with seas of applications [1]. The fundamental features of the system attract thousands of scientists and researchers into the field due to the potential impact offer by the technology [2]. Till date, various applications have been demonstrated using droplets as a chemical microreactor [3], single cell analysis [4], drug screening [5], particle synthesis [6], food engineering [7], high throughput biological assays [8], protein crystallization [9] and DNA amplification [10]. The list of applications goes on and is extensive. But it is apparent that what one could do with droplet microfluidics is boundless and immeasurable. The interest in active droplet generation stems from the possibilities of extending the current usage to a whole new level and bringing on many more other novel applications. Active droplet generation increases the robustness of the system and offers greatly flexibilities in fluid manipulations. It also allows a much more complex operation to be performed at a greater ease due to the integration of an active element which offers an additional degree of control. Active droplet generation offers the solution for the realization of a complex lab-on-a-chip operation [2] which requires a greater level of fluid control.

The second point is the ability to generate droplet on demand and exquisite control over each individual droplet generated. This unique form of control and manipulation can only be achieved by the use of active droplet generators. This ability stretches immensely on what one could do with droplets and also opens up the doors to many other possible applications. In summary, active droplet generators offer many distinctive advantages which appeals to users in different ways. The interest in active droplet generators is appealing and a topic worthwhile for investigation. 


\subsection{Droplet generation and manipulation}

The problem that we are addressing is limited to droplet generations in an enclosed microfluidic device where the fluids are inside enclosed microchannels. We confronted only two phase flow conditions where two immiscible fluids are used to form droplets. In these conditions, we examine the difference between different passive and active droplet generation methods. We conclude by examining the needs for a dynamic and robust method of active droplet generation as a tool for different bio-chemical applications.

\subsubsection{Passive droplet generation}

Passive droplet generation is defined here as the formation of droplets by only hydrodynamic means. Droplets are formed by flowing two immiscible fluids into microfluidic devices either by using flow rate control syringe pumps or controlling of inlet pressures via pressure sources. A detailed study on the differences between the two fluid delivery systems can be found in the following work [11]. In order to prevent droplet coalescence and condition favorable to droplet generation, surfactants are often added into either one of the two fluids. In brief, the use of surfactants decreases the interfacial tension between the two fluids and allows droplets to be stored without coalescences for a much longer period of time. The use of surfactants is also useful for many bio-chemical applications which allow droplets to be re-injected into devices for subsequent manipulations [12, 13].

In passive droplet generation, four of the commonly used geometries are T-junction [14, 15], flow focusing [16-18], co-flowing [19-21] and head-on [22, 23]. The droplet generation mechanism is similar in a way but yet distinctive differences can be observed. Each of the geometry possesses its individual uniqueness. Different droplet formation regimes and non-trivial scaling laws are derived for each of the geometry. Figure 1.1 illustrates each of the commonly used geometry. 

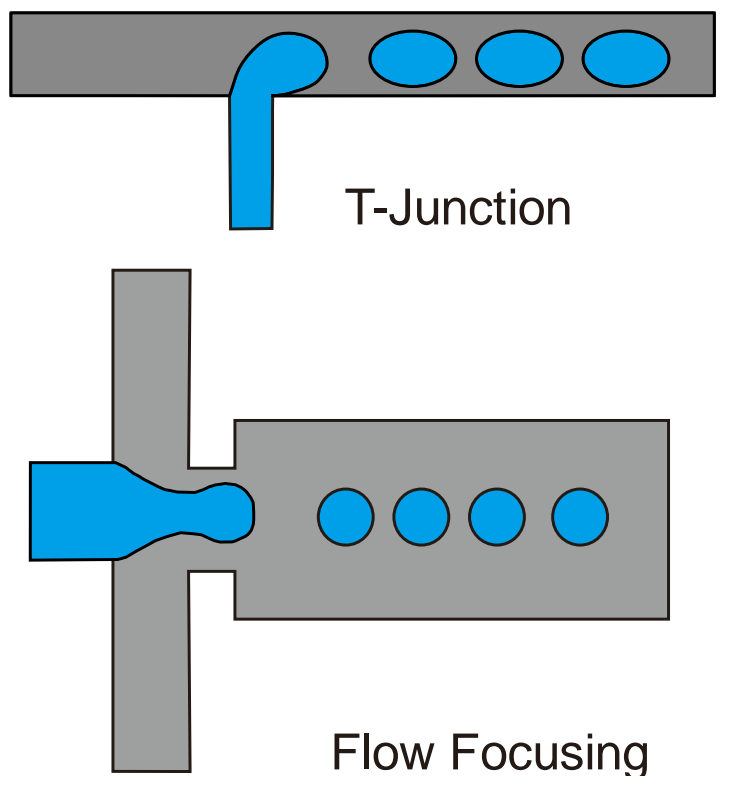

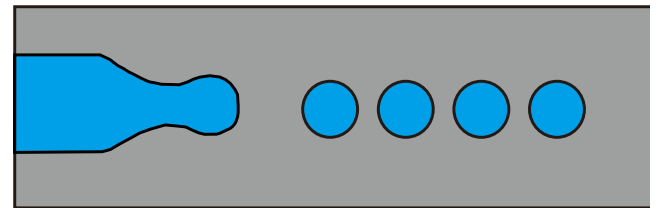

Co-Flowing

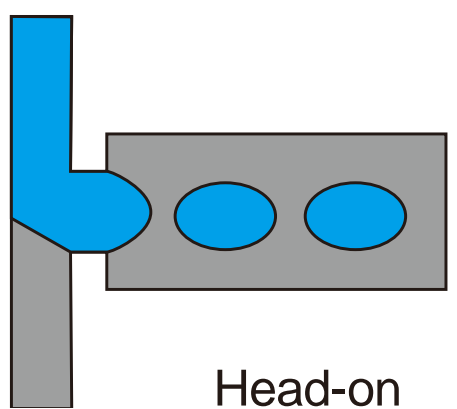

Figure 1.1: Schematic sketches of the commonly used droplet formation geometries. The blue region represents the dispersed phase fluid and the grey region represents the continuous phase fluid.

In the above mentioned droplet formation geometries, the size of the droplets formed can be predicted by balancing the Laplace pressure of the dispersed phase fluid with the shear force [15] exerted by the continuous phase fluids using equation (1.1):

$$
r \sim \frac{\sigma}{\eta \dot{\varepsilon}}
$$

where $r$ is the radius of the droplets formed, $\sigma$ is the interfacial tension between the fluids, $\eta$ is the viscosity of the continuous phase fluid and $\dot{\varepsilon}$ is shear rate which can defined as (1.2):

$$
\dot{\varepsilon} \sim \frac{2 v}{y_{0}}
$$


where $v$ is the velocity of the fluid and $y_{0}$ is the channel radius at the center of the flow estimated by triangular approximation.

Substituting equation (1.2) into (1.1), we get:

$$
r \sim \frac{\sigma y_{0}}{2 v \eta}
$$

From the above simple equation (1.3), it can be deduced that the radius of the droplet is a function of the interfacial tension, geometry of the channels, flow rates of the fluids and the viscosities of the fluids. However, this simplified predication does not accurately describe the complex flow behavior. The predicated droplet diameter and the measured diameter deviates by a factor of two when compared by Thorsen et al. A more sophistical and non-trivial scaling law can be obtained from these various literatures [24-26]. These scaling laws take into account the volumetric flow rate ratios, viscosity ratio between the fluids and the aspect ratios between the channel dimensions.

In the above, it is apparent that passive droplet generators have limited control over size of the droplet formed once the fluids and the geometries of the channels are fixed [27]. Although it can be argued that physical properties such as the interfacial tension $\sigma$ and $\eta$ can still be varied with the use of different concentrations or types of surfactants [28] and by adding glycerol [29] into fluids, this is often non-desirable due to the specific needs of different applications. Hence in such conditions, the sole mode of changing the size or frequency of droplet generation is limited to manipulating the flow conditions, i.e., changing the applied volumetric flow rates or inlet pressures. This limitation warrants for the need of active droplet generator which provides more flexibility on the control of droplet size and frequency generated in a microfluidic device. 


\subsubsection{Active droplet generations}

Active droplet generations here refer to the use of external perturbations to "modify" the flow system and thus providing an additional means of control over the droplet generation in microfluidic devices. Often, active droplet generations are achieves through modifications of the flow parameters, interfacial tensions or the viscosities of the fluids with the use of an external source. External sources demonstrated till date includes thermo-capillary [30-34], pneumatic [35-37], piezoelectric [38], magnetic [39-41], electric [42-49], acoustic [50-52] and optic [53]. The working principle of each external source is different and unique.

Active droplet generation provide several distinct advantages over the passive counterpart. Firstly, the use of external sources allows a more robust, flexible and dynamic control on the droplets generated. For example, external sources can be easily turned on and off depending on the user needs and the required application. The ability of active control on demand opens up different possibilities for many different applications. Secondly, the use of external sources also allows the frequency of the droplets to be manipulated at ease. The frequency of the droplets controls the velocity of the droplets which in turn determine the processing time within a specific reaction. Actively manipulating the frequency of the droplets allows users to tune towards velocity of the droplets to meet the requirement of the process. Droplets are also often used as an individual "micro-reactor" in droplet based microfluidics. In such a scenario, the size of the droplets determines the sensitivities of the encapsulated content to be analysed. The use of an active control allows a greater flexibility when encapsulating biological materials of different sizes. Last but not least, active droplet generation also allows droplets to be produced at a faster rate when the accessible flow rates are limited by geometrical constraint such as the size of the orifice or the fluidic pressures that the device can withstand. The ability to produce droplets at a faster rate is desirable for many applications which require high throughput droplet production.

On the other hand, the active droplet generation has also several disadvantages. Firstly, the use of external sources inherently implies that additional equipment is needed to be incorporated into the system. The use of external sources increases 
not only the complexity of the operation but also the cost needed to run the process. For example, in electric field mediated experiments [54], a function generator is needed to produce the electrical signal for the droplet manipulation. In order to create comparable electric field strength, this is often coupled to a high voltage power amplifier to magnify the signal. Secondly, some external sources of actuation require complicated fabrication methodology which requires high level of engineering skill sets. In thermally mediated droplet generation [55], micro-heater and sensors are integrated into passive droplet generators to induce localised heating and temperature sensing. The fabrications of such devices are labour intensive as it requires long man hours in the cleanroom to deposit the metals and also careful alignment of the micro-heater and sensors at the junction of droplet formation. Last but not least, active droplet generators introduce additional complexity into the already complicated flow behaviour of the fluidic system. Comparing with the passive systems which is well understood and studied, the droplet formation mechanism and scaling laws are often briefly explained and tackled. The ambiguity in understanding of the system is a major shortcoming which in turn decreases the popularity of implementing external actuating sources into passive droplet generators.

Nevertheless, the use of active droplet generators still remains as an attractive solution for many research applications and the realization of complex lab-on-chip operation which require greater control and flexibility in fluid manipulation. The exhaustive list of benefits offered by different active method of control justified the usage and incorporation into different system. It is also apparent the benefits of active droplet generators will be an enticing alternative in the upcoming days. 


\subsection{Fluids interaction with an electric field}

\subsubsection{Electrohydrodynamic concept}

The concept of electrohydrodynamic (EHD) is due to the interaction between electric fields and fluids. The difference in electrical properties between the fluids affects the force balance at the fluid interface which acts to either stabilize or destabilize the interface [56]. The electric field induces electrical stresses at the interface between the fluids due to a conductivity or permittivity gradient. To generate the electric field, different approaches have been developed using either a direct current (DC) or alternating current $(\mathrm{AC})$ voltage source. Electrodes of different geometries and size have also been demonstrated using the concept of EHD. However, aspects relating to droplet generation will be the main focus here to limit the discussion since vast amount of literatures are available and not within the scope of the thesis.

Currently, two different techniques are employed using EHD to produce droplets. Monodispersed droplets or droplets with a wide range of sizes are produced either by electrospraying or single droplet generation [57]. In both methods, droplet generations are often due to the accumulation of charges at the interface of the fluid. Droplets are generated when the interfacial charges overcome the capillary pressure. Figure 1.2 illustrates the droplets formed by electrospraying.

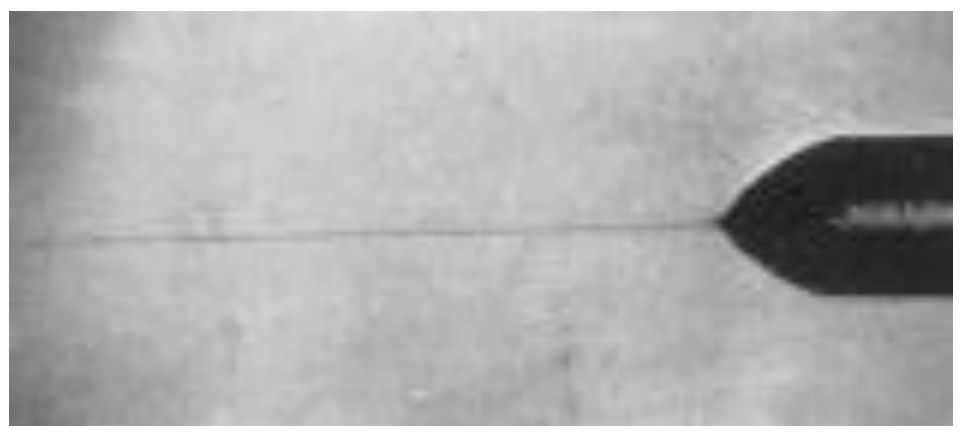

Figure 1.2: First experimental photo by John Zeleny in 1917 depicting electrospraying of droplets due to the instabilities of electrified liquid surface [58]. 
This phenomenon was later further described and quantified by Sir Geoffrey Taylor in the year 1964. In the article [59], he first proves that the EHD instabilities arises due to the pressure difference across the droplet interface which invalidate the previous assumption made by Zeleny that the instabilities arises when the pressure inside and outside the droplet is the same. The article also shows that the formation of the cone-jet or Taylor's cone is the onset of the electrospraying process. The Taylor's cone was measured to have a semi-vertical angle of $49.3^{\circ}$ as shown in figure 1.3.

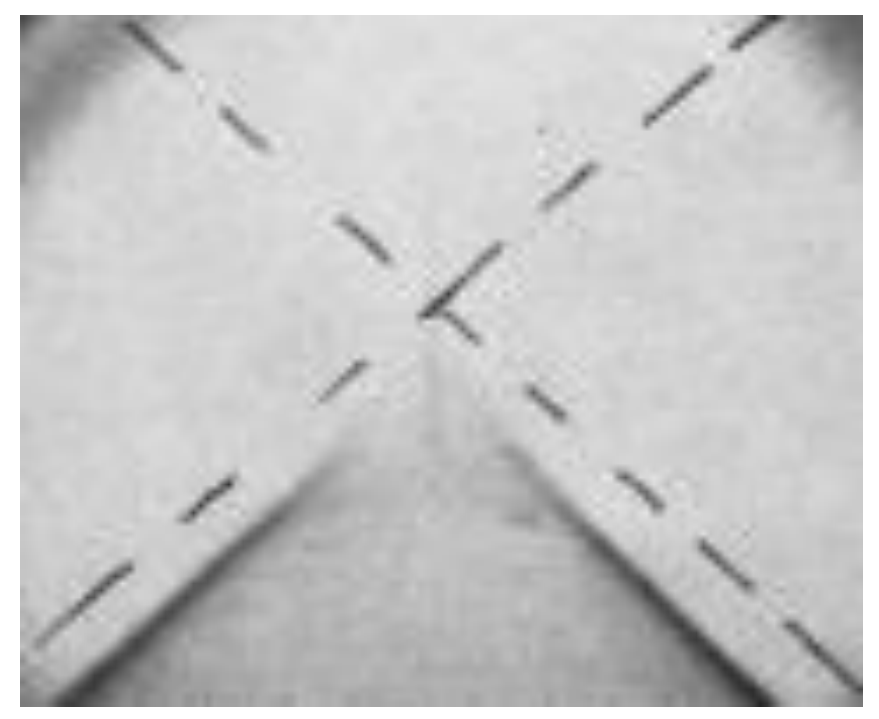

Figure 1.3: Formation of the Taylor's cone[59]. The broken lines are at an angle of $98.6^{\circ}$.

Following his works, a large number of research have been done to elucidate the behavior with both DC and AC electric fields capitalizing on the interest for nanotechnology [60].

Electrohydrodynamic techniques are also employed for single droplet generations to produce droplets on demand [57, 61]. Using a similar mechanism, highly monodispersed droplets in the range of micro and pico liters with standard deviation less than $2 \%$ can be produced. 


\subsubsection{Electrocapillarity}

Electrocapillarity phenomena are observed in various phenomenon such as electrowetting $(E W)$, continuous electrowetting $(C E W)$, electrowetting on dielectric (EWOD) and electric double layer (EDL). It was first investigated by Lippmann in 1875 , in which he observed a capillary rise effect due to the change in surface tension under an electrical effect [62]. The term was later coined based on the two observed effects. Building upon his findings, he invented the electrometer (Figure 1.4) which was later used in the first electrocardiography (ECG) machine.

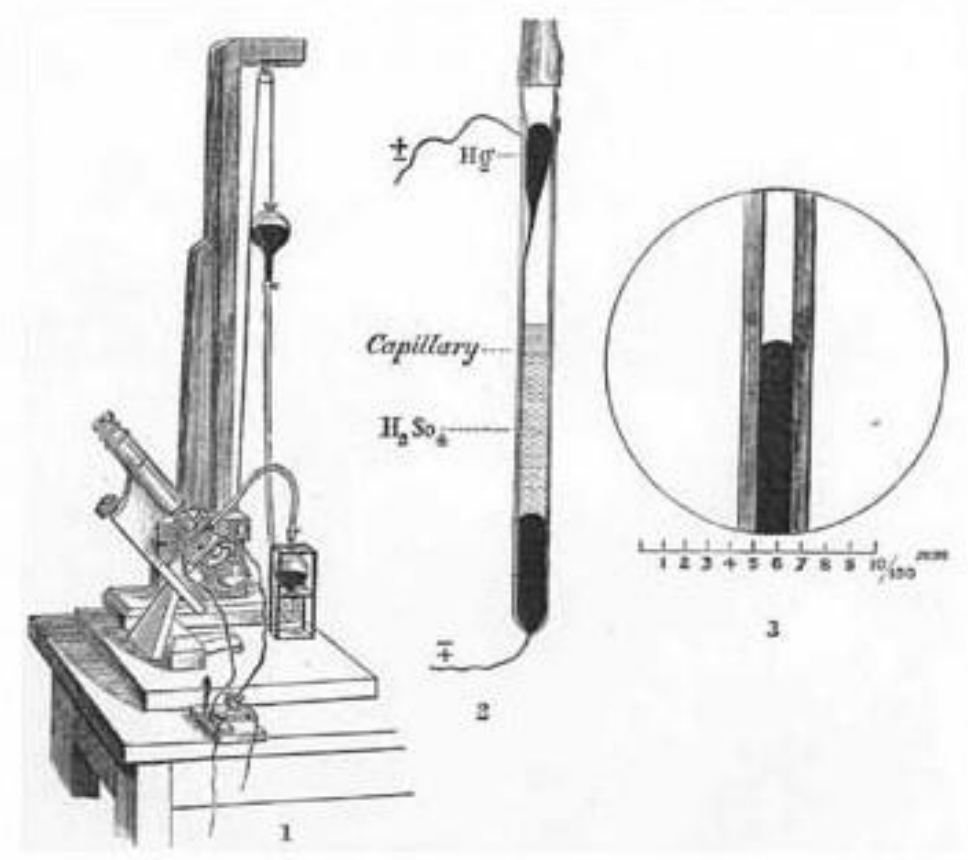

Figure 1.4: Drawing of the lippmann's electrometer.

In a boarder sense, electrocapillarity can also be defined as a change in the interfacial tension with an applied potential difference [63] which can also occurs at the interface between immiscible fluids. Watanabe investigated extensively the electrocapillarity phenomena at oil-water interfaces with different types of fluids and surfactants [63-65]. He found that the interfacial tension between the fluids changes 
with the applied potential difference and this is dependent on the type of fluids and surfactants used.

In retrospect, the combination of these effects can be applied to the generation of droplets in microfluidic devices via the electric bond number [66] and the capillary number described previously. The electric bond number describes the deformation of droplets due to an applied electric field which is opposed by the interfacial tension can be expressed as:

$$
B o_{e}=\frac{\varepsilon\left|E^{2}\right| r}{\gamma}
$$

Where $\varepsilon$ is the electric permittivity of the surrounding fluid, $E$ is applied electric field strength, $r$ is the radius of the droplets and $\gamma$ is the interfacial tension between the fluids.

In the above proposed dimensionless number, most studies focus on using a DC voltage induced electric field and investigating the effects on the deformation of droplets. Relatively few studies are done using an AC voltage induced electric field. Detailed experimental studies on this are essential to enhance the understanding on this topic. Both the proposed dimensionless numbers (Electric bond number and Capillary number) and the correlationship between them will be the focus of this thesis. The coupling and interaction of an AC electric field with the droplet formation mechanism in a flow focusing configuration will be explained in detailed in the later chapters. 


\subsection{Thesis subject}

In the following chapters, we will use the variation of the AC voltage to study the influences on the droplet formation in a microfluidic flow focusing device. This novel method of active control has not been studied or demonstrated before. In a first part, we will study how such variations are influences by various parameters such as flow rates, conductivities of the fluids, frequencies and electrode connections. In the second part, we investigate the dynamics of the system by looking into the response times of the system and the usage to play different musical notes. We also demonstrate for the first time the use of such fast and reliable control to play different musical sound tracks in the microfluidic jukebox that we developed. 


\section{Part 2: Static studies}

\section{Chapter 2: Concept}

Say Hwa Tan, Benoit Semin and Jean-Christophe Baret

Patent Application number: PCT/EP2012/001423

\section{Contents}

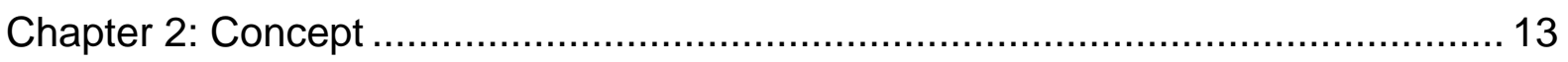

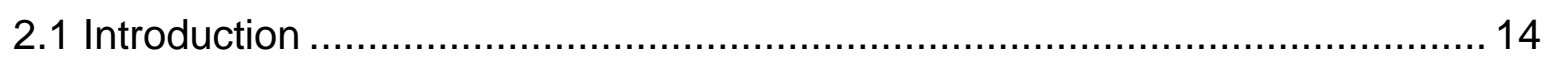

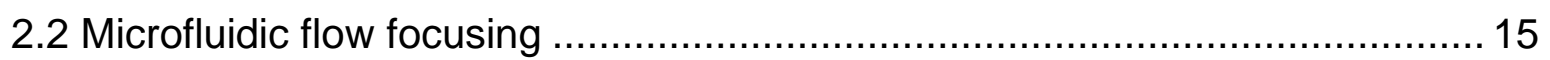

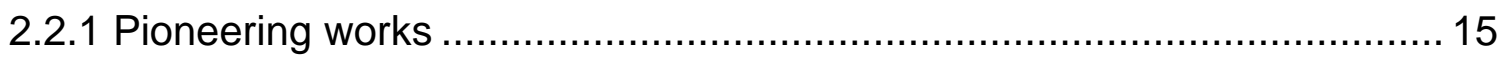

2.2.2 Three dimensional (3D) microfluidic devices ....................................... 16

2.2.3 Geometrical variations of flow focusing ............................................. 18

2.2.4 Droplet formation Regimes ……….............................................. 20

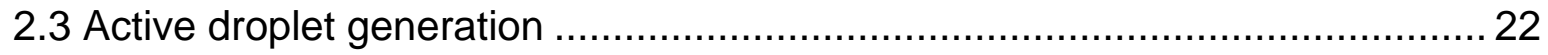

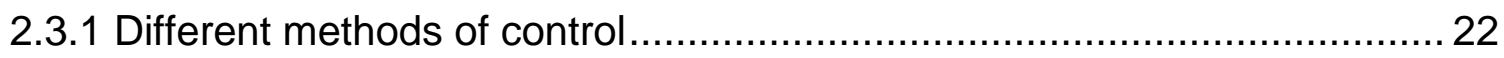

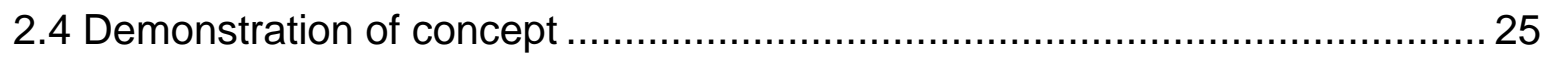

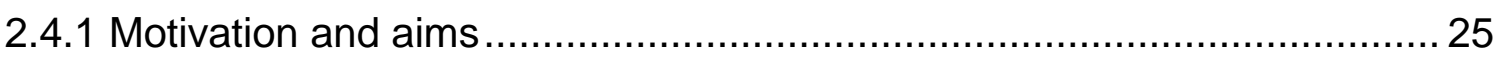

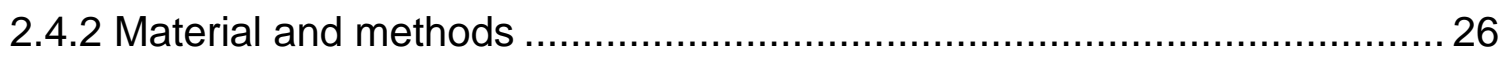

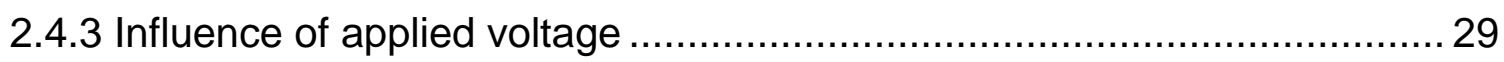

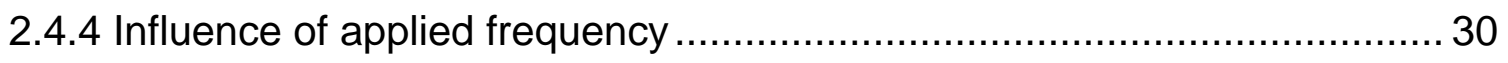

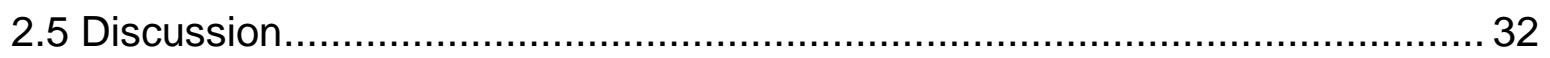

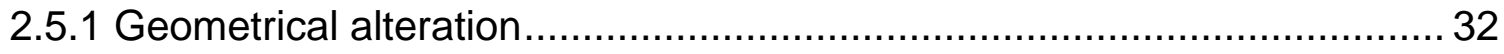

2.5.2 Other factors to be considered............................................................. 34

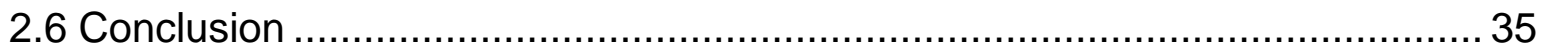




\section{Chapter 2: Concept}

\subsection{Introduction}

The use of microfluidic devices to generate droplets was first introduce by Thorsen et al. in 2001 [15]. However, it was the advent of soft lithography techniques which allow easy access to this field and thus contributing to the widespread usage. Till date, polydimethylsiloxane (PDMS) it still widely used as a replicating material to fabricate microfluidic devices $[67,68]$. This is mainly due to reasons such as low cost of production and fast turn over time for conceptual testing. As mentioned previously, four different types of geometries are commonly used to produce droplets in microfluidics using PDMS to fabricate microfluidic devices. Among them, the flow focusing geometries is regarded as the preferred choice as it is relatively easier to use to form droplets. The flow focusing was introduce by both Anna et al [16] and Dreyfus et al [18] in 2002. Droplets are formed by first focusing a fluid in the center channel and then a second immiscible fluid flowing at both the side channels exert pressure and viscous stresses to deform the interface. Different variations of the flow focusing geometries have evolved with time and many detailed studies have been done to elucidate the complex flow behavior [24-26].

Different types of active droplet generators have been developed to overcome the limitations of the passive counterparts. One such limitation is the size of the orifice in the flow focusing configuration. In such a configuration, the size of the droplet produce depends strongly on size of the orifice which limits the flow of the fluids. In the following, we will first review the flow focusing geometry to understand the complex nature of the droplet formation mechanism, different geometrical variations, proposed scaling laws and also the importance of the wetting abilities of the fluids. Different types of active generators are also reviewed and compared to unravel the interest on using an AC voltage induced electric field which is the main motivation of this thesis. Understanding all of the above, it will provide a foundation for the basis of this thesis and also develop the experimental approach to adopt to tackle the scope. 


\subsection{Microfluidic flow focusing}

\subsubsection{Pioneering works}

The use of a microfluidic flow focusing device to generate monodispersed droplets results in a paradigm shift of droplet production from a "top down" to a "top up" approach [16]. This shift stems from the ability to create droplets of highly precise volumes at extremely high throughput. Two factors undermine the stable production of droplets from an "ordered" to a "disordered" state [18]. Firstly, the wetting of the fluids with channel walls determines whether stable oil-in-water $(\mathrm{O} / \mathrm{W})$ or water-in-oil (W/O) droplets can be formed [25, 26]. Secondly, the concentration of surfactants in the fluids has to be well above the critical micelle concentration (CMC) to maintain a stable interface which promotes droplet generation [18].

Using a hydrodynamically focused thread, $\mathrm{Xu}$ et al demonstrated the precise control on droplets generated. Highly monodispersed droplets with polydispersity of less than $1 \%$ can be generated [69] with a silicon microfluidic device. However, the breakup of the center dispersed fluid thread depends on a number of factors such as the interfacial tension, viscosity and geometry of the channel. The physics and the breakup mechanism of the droplet were not well explained in the manuscript. Figure 2.1 illustrates the ability to form uniform droplets formed at different flow conditions using such method.
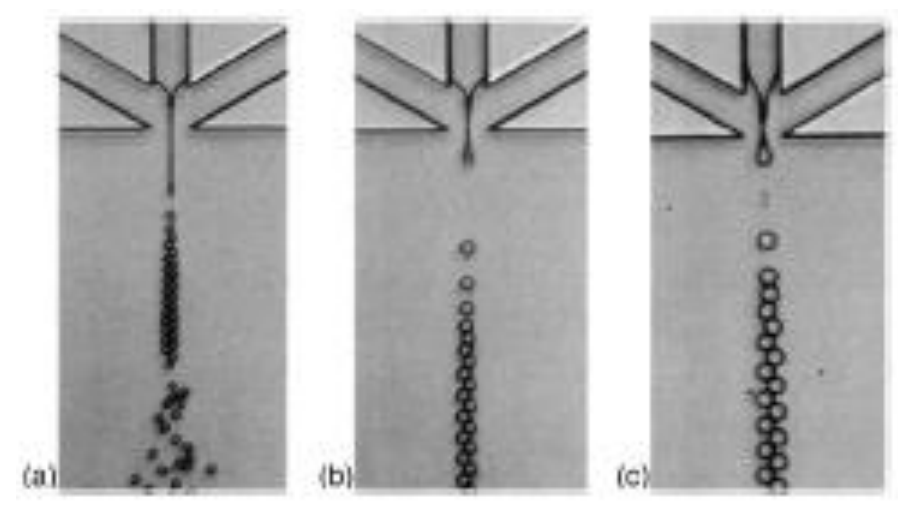

Figure 2.1: Generation of monodispersed droplets at different flow conditions [69]. 


\subsubsection{Three dimensional (3D) microfluidic devices}

Several groups proceed on to enhance the performance of droplet generation with sophisticated fabrication techniques and microfluidic devices. This is achieved through the use of a three dimensional (3D) orifice or devices which focused the droplet breakup at the orifice. In this way, maximal shearing stress is exerted on the dispersed phase fluid. This assisted the formation of droplets as the flow of continuous phase fluid is now able to encapsulate the dispersed fluid. Takeuchi et al first uses glass capillaries to create an axisymmetric flow-focusing microfluidic device (AFFD) in PDMS [70]. This allows polymer coated droplets to be generated for microencapsulation purposes. He suggested that this method is more superior than the two dimensional (2D) microfluidic generators as this avoids the problem of the dispersed fluid wetting or adhering to the channel walls. Huang et al then proposed using a three layered SU-8 microfluidic device to create the 3D microfluidic channels [71]. The main advantage of his system over the AFFD is the eliminated of the need of alignment of the capillaries and also the flexibility in the channel dimension. $\mathrm{He}$ also demonstrated that his system can be used in both open and closed configuration generating both $\mathrm{W} / \mathrm{O}$ and $\mathrm{O} / \mathrm{W}$ droplets. However, physical insights to his system were not investigated thoroughly.

Yobas et al used a cusp-like edge orifice to investigate extensively the difference between the 2D and 3D microfluidic flow focusing devices [72, 73]. The 2D device was fabricated in PDMS using convention soft-lithography technique and the 3D was fabricated by reactive ion etching (RIE) of silicon. Pure fluids without surfactants are tested to allow accurate measurements of the interfacial tension between the fluids. A computational fluid dynamic (CFD) simulation was also done to understand the complex flow behavior during droplet generation. Both experimental and simulation results show large improvement in the droplet generation rate. Droplets can also be formed in the range of about $10 \mathrm{kHz}$ or higher as the 3D devices allow higher flow rates to be used as compared to the $2 \mathrm{D}$ devices. The authors also observed a delayed in the onset of the jetting regime and droplets formed mainly in the dripping regime at a wider range of flow rates. Theoretical modeling predicting the diameter of the droplets and droplet generation frequency was also proposed and compared with experimental results. The proposed models fit well with the experimental data when 
the droplets are formed in the dripping regime and deviates when the droplets are formed in the jetting regime. This is mainly because droplets formed in the jetting regime are mainly due to Rayleigh plateau instabilities [74]. Figure 2.2 and 2.3 below illustrates the 2D and 3D microfluidic devices used.

(a)

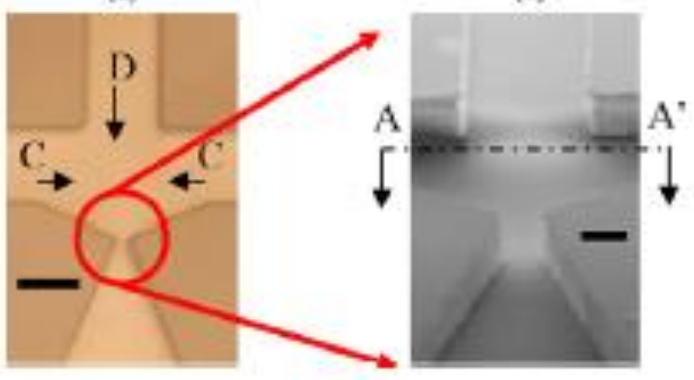

(c)

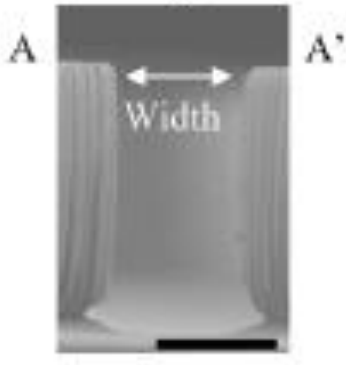

Figure 2.2: (a) 2D flow focusing in PDMS, (b) \& (c) are the SEM images [72].

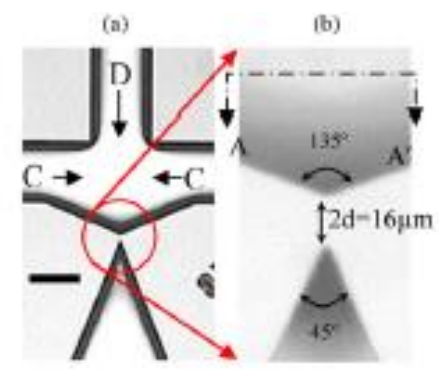

(c)

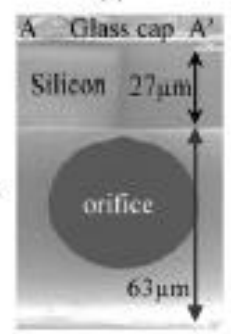

(d)

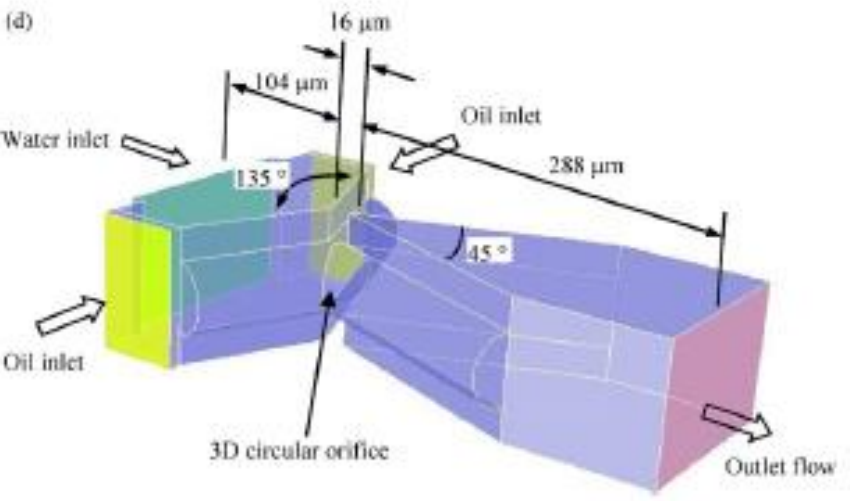

Figure 2.3: (a) to (c) are the 3D flow focusing device and (d) simplified sketch of the 3D geometry used for the CFD studies [72]. 
It is apparent that 3D microfluidic flow focusing offers several distinct advantages over the 2D counterparts. However, the usage of such devices is still not widespread and preferred. This is probably due to the complex fabrication technique that is needed to master before one can effectively implement for usage.

\subsubsection{Geometrical variations of flow focusing}

The performance of droplet generation in microfluidic devices depends strongly on the geometrical designs of the microfluidic channels. In brief, the designs of the microfluidic channels affect the flow profile of the fluids and location of the droplet breakup. Indirectly, the accessible range of capillary number which determines the dominant mechanism of droplet generation and the rate of droplet production are also affected. Abate et al studied the impact of the inlet geometry in droplet formation by varying both the channel widths and angle of injection [75]. He observed that Tjunction droplet makers produce droplets at low and moderate capillary number. On the other hand, flow focusing droplet makers produce droplets at moderate and high capillary number. The observation occurs when he used immiscible fluids with viscosity ratio of about 1 . This stark contrast between the two is due to the wall effect in the T-junction configuration which smooths out the instabilities and suppressed the formation of droplets. As a result, the jetting regime is suppressed and the formation of a parallel flow between two immiscible fluids is induced. The experimental results also suggested that the use of the flow focusing geometry would enable the production of a larger amount of droplets which is useful for high throughput applications as droplets can be formed at higher capillary numbers.

Several geometrical variations of the flow focusing configuration have since evolved throughout the years. Tan el at introduces the expanding nozzle to create a velocity gradient at the orifice [76]. This enables the break of droplet to occur right at the orifice where the velocity is greatest. Chen et al complement the idea by using a converging and diverging nozzle [77] at the junction of droplet formation. He found that a greater control on the size of the droplet is achieved with the modification and the size of the droplet formed can also be reduced significantly. This is also dependent on the dimension of the orifice which affects the shearing rate on the 
dispersed phase fluid. On the contrary, several works also focused on using straight cross junctions to study the complex nature of droplet formation [75, 78-80]. Cubaud et al uses a square geometry to investigate extensively the formation of $\mathrm{O} / \mathrm{W}$ droplets using fluids with different viscosities and interfacial tension. Tan et al uses a symmetric cross flow but with different inlet/outlet widths to study the formation of both $\mathrm{O} / \mathrm{W}$ and $\mathrm{O} / \mathrm{W}$ droplets. In both studies, different scaling laws, flow regimes and the effect of fluid properties are also presented and discussed. Several numerical simulation works $[79,81]$ have also been done to elucidate the scaling laws and the dependence of the size of the droplets on both the capillary number and flow rate ratio.

On a broad perspective, geometrical variation of the flow focusing can be classified into two types, mainly with and without the orifice. Figure 2.4 illustrates the schematic sketch of both cases. In the case of without the orifice, the geometry is often referred also as the cross flow configuration. In this geometry, the flow of the dispersed fluid is accelerated towards the junction and reaches a maximum at the entrance of the outlet channel. The flow profile is then maintained throughout the straight channel. On the hand, for the case of with the orifice, the constriction at the junction changes the flow profile of the fluids. Fluids are accelerated and "squeezed" at the orifice and thus producing droplets of smaller size. The flow profile at the outlet also changes due to the expansion in the channel which slows down the flow of the droplets.
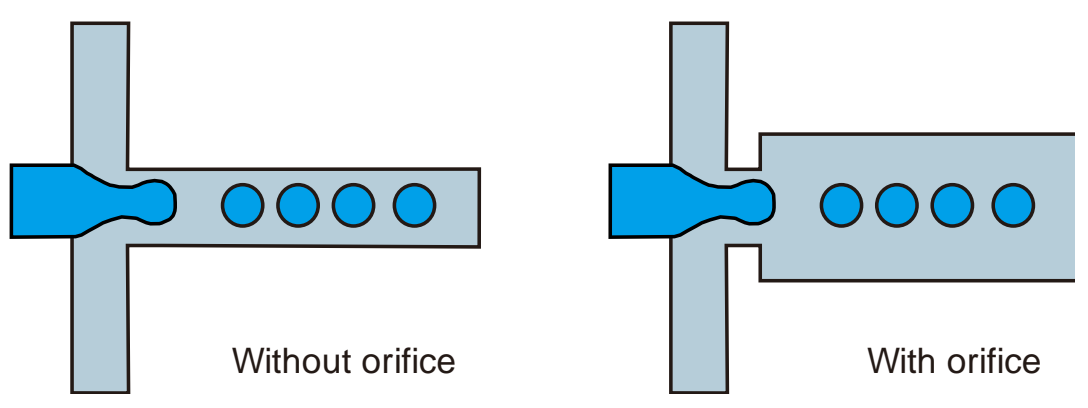

Figure 2.4: Schematic sketch of two main types of the flow focusing geometry. 
Nevertheless, the understanding on the intrinsic differences between each geometrical variation will allow the selection of the most appropriate geometry to use for this study.

\subsubsection{Droplet formation Regimes}

The formation of droplets in microfluidic device is characterized and can be described by distinctive droplet formation regimes. The droplet formation regimes illustrate the location of the droplet breakup, the dominant stresses involved in the breakup process, relative size of the droplets and non-trivial scaling laws predicting the droplet volume $[17,25,26,78]$. In order to quantify the regimes, often either the capillary numbers or the flow rate ratios are calculated and used. However, these numbers often act only as a gauge and reference. The critical capillary number dictating the transition between each regime differs slightly depending on the dimensionless variables of the system, channel designs and also the physical properties of the fluid.

In microfluidic flow focusing, Anna et al identified four different droplet formation regimes. The four main droplets formation modes are geometry-controlled breakup or squeezing, dripping, jetting and thread formation. In brief, the squeezing regime occurs at low capillary number where droplets are the neck of the dispersed fluid is squeezed between the confinements of the channel walls. The dripping regime occurs at moderate capillary number where droplet breakup is due to mainly the shear stress exerted by the continuous phase fluid. In jetting, droplets are formed at high capillary numbers and droplet breaks up via Rayleigh plateau instability further downstream of the channel. The thread formation regime occurs at low capillary number and only with the presence of large amount of surfactants. Figure 2.5 and 2.6 depicts the different droplet formation regimes occurring at different capillary number and flow rate ratio [17]. 
(a)

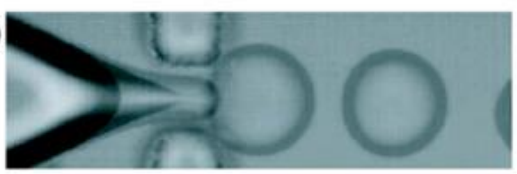

(c)

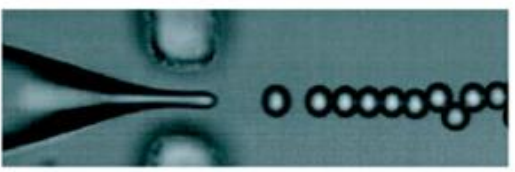

(b)

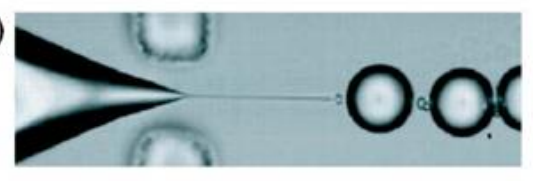

(d)

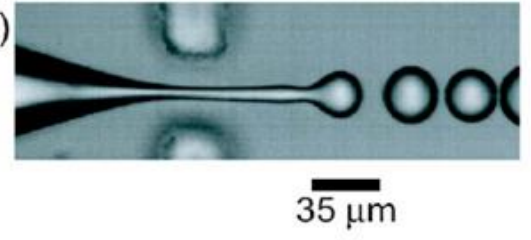

Figure 2.5: Illustration of the different droplet formation regime. (a)Squeezing regime, (b) thread formation, (c) dripping and (d) jetting[17].

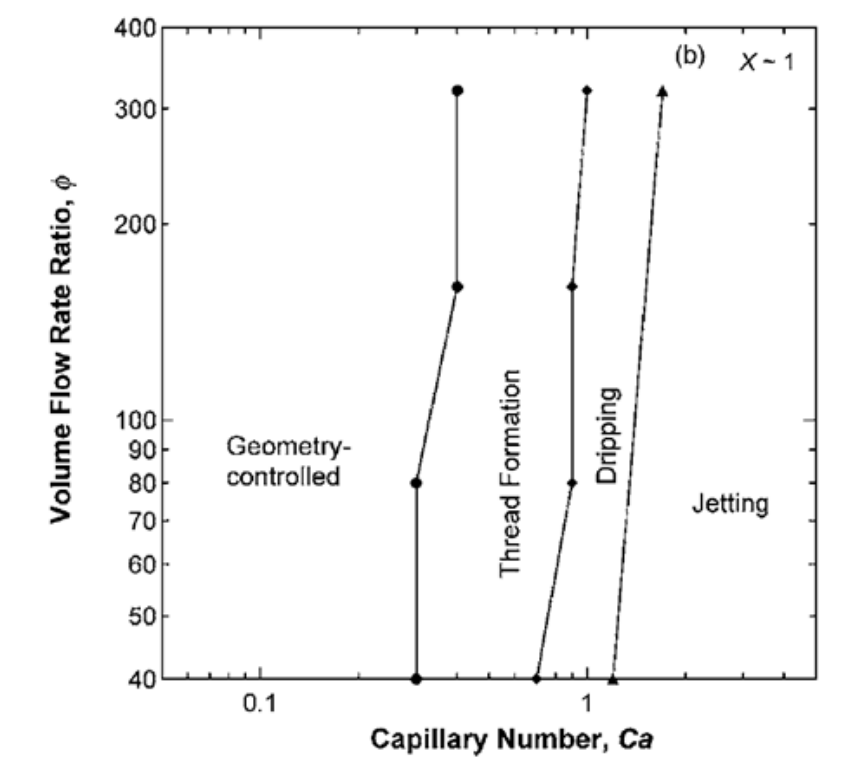

Figure 2.6: Different droplet formation regimes with respect to the flow rate ratio and capillary number[17].

In the absence of surfactants and the formation of viscous droplets, Cubaud et al also observed similar droplet formation regimes occurring at different capillary numbers. He observed additional modes of droplet breakup such as threading, tubing and viscous displacement. A flow map illustrating the different droplet formation regimes at different capillary numbers is also presented [78]. 


\subsection{Active droplet generation}

\subsubsection{Different methods of control}

Different methods to actively control the size of the droplets formed in microfluidic devices have been developed in the past decade. With the increase in complexity in lab-on-a-chip applications, these active methods provide robust solutions to meet the raising demands. The size of the generated droplets can be manipulated using different means such as pneumatic control [35-37, 82], piezoelectric actuation [38, 50], surface acoustic wave [51, 52], light sensitive surfactants [53], magnetic control [39-41], thermo-capillary manipulation [30-34, 39, 83] and electro-capillary manipulation [42-49].

In the above, electrically mediated droplet generation is one of the most robust, reliable and dynamic method to control the size or frequency of the droplet formed. It offers several distinct advantages over other systems. For example, in pneumatic assisted droplet formation, the long term reliability and performance may be a concern as the "stretching" of PDMS may deteriorate with repeated usage. In such a device, the sizes of the droplets formed are often manipulated by deforming the interface between the fluids through "stretching" of the PDMS material. This is often achieved by using an integrated valve or wall actuated by a pressurized air source. The size of the droplets formed in such cases depends on the applied pressure and also the control signal applied. The responsive time of such a system is also relatively longer than the electrical methods.

Electrically mediated droplet generations also appeals to chemical and biological applications which are both temperature and contaminant sensitive. For example in magnetically assist droplet formation, often the use of magnetic responsive fluids such as ferrofluid may contaminate the biological or chemical reagent which interfere the reaction process. Thermo-capillary mediated droplet generation is also not suitable for temperature sensitive applications such as the polymerase chain reaction (PCR) which is used to duplicate genetic materials $[84,85]$. 
On the hindsight, the current electrical methods proposed also have its limitations and disadvantages. Electrical control in droplet microfluidics was first proposed by Link et al in 2006 [48]. He uses the concept of electrostatic charging of the aqueous fluid to generate electrical forces to assist the droplet formation. This is achieved by integrating indium tin oxide (ITO) electrodes between the junctions of droplet formation. The size of droplets generated decreases with the increase in applied DC voltage. However, this method has several drawbacks. Firstly, the contact of the electrodes with the fluids results in electrolysis at high voltages. As a result, bubbles are formed during the electrochemical process which destabilized the production of monodispered droplets. Figure 2.7 illustrate the formation of bubbles observed when similar experiments were repeated during the doctorate tenure.
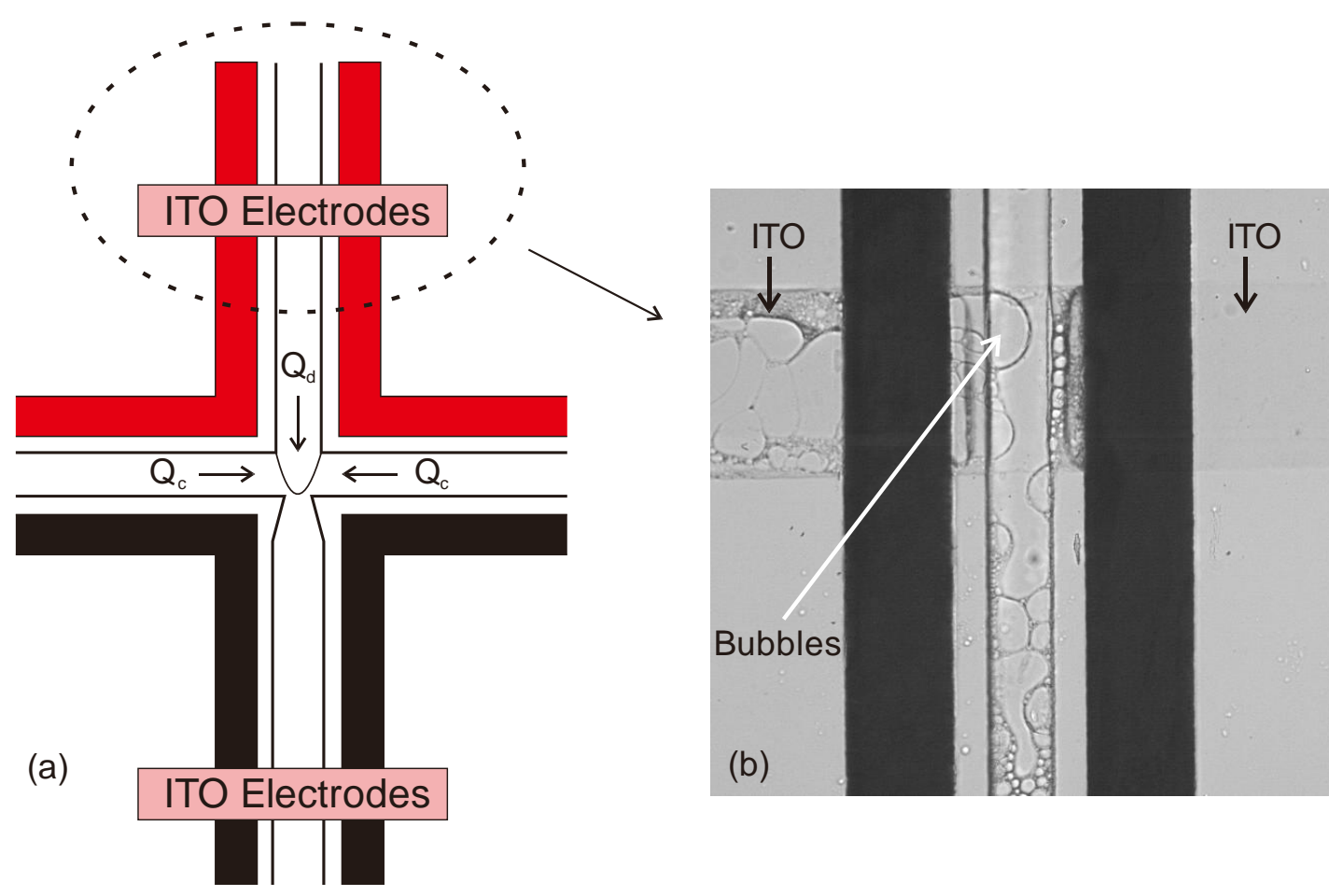

Figure 2.7: Formation of bubbles due to electroylsis. (a) Schematic sketch of the microfluidic device geometry with the electrodes and (b) formation of bubbles at $1000 \mathrm{~V}$. The $L$ shape electrodes provide the connection to the voltage source. $Q_{c}$ and $Q_{d}$ refers to the volumetric flow rate of the continuous and dispersed phase fluid. 
The charging of the aqueous fluid also results in the production of highly charged droplets. The droplets may undergo further columbic reactions after the production which results in droplets of high dispersion. Another drawback of such method includes the need of alignment of the ITO electrodes with respect to the microfluidic channels. This problem is eliminated in the subsequent work by Kim et al [47] with the insertion of electrodes at a fixed location within the microfluidic channels. However, the problem of electrolysis at high voltages may persist as the electrodes are in contact with the aqueous fluid.

Electrowetting on dielectric (EWOD) is another approach developed by Mugele et al to manipulate the generation of droplets in microfluidic devices. The difference between electrowetting (EW) and EWOD is the addition of a dielectric layer which allows the use of higher voltages due to the insulation provided. In this approach, either an AC or DC voltage can be used to tune the apparent contact angle of the aqueous fluid with the channel walls. The size of the droplets produce depends on the dimensionless EW number and the EW equation which are describe in equation (2.1) and (2.2) below.

Electrowetting equation:

$$
\cos \theta=\cos \theta_{Y}+\eta
$$

Where $\theta$ is the water contact angle, $\eta$ is the dimensionless EW number and $\theta_{Y}$ is the young angle.

Dimensionless EW number:

$$
\eta=\frac{C U^{2}}{2 \sigma}
$$


Where $C$ capacitance due to the dielectric layer, $U$ is applied voltage and $\sigma$ is the interfacial tension between the fluids.

Although the physics and mechanisms governing EW or EWOD is well described and explained in various works [42-45], this method of control requires exquisite fabrication technique of the dielectric layer. The dielectric layer which is in the order of a few micrometers is often hard to control as it is fabricated by dip coating process.

\subsection{Demonstration of concept}

\subsubsection{Motivation and aims}

As mentioned, the use of electric field provides the most robust, dynamic and reliable means of control in generation of droplets in microfluidic devices. Although several electrical methods have been proposed previously, a reliable method with simple fabrication technique is still not available. This impedes the use and implementation of electrical control elements for application based processes. Hence, the motivation for this thesis is to develop a reliable method of electrical control which is relatively simple to fabricate and also easy to implement. A secondary motivation factor for this work will be the use of electrodes which are not in contact with the fluids to eliminate the problem of electrolysis.

The aims of this research is focus primary on the use of an $A C$ voltage induced electric field to control the size or frequency of the droplets formed. Unlike the DC voltage counterpart who is widely investigated, the use of an AC voltage induced electric field to control the size of the droplets is still poorly understood. This work also aims to explain different observed complex phenomenon with simple physical scaling laws such as the capillary number and electrical bond number.

The work coupled the flow focusing geometry with the use of an AC voltage induced electric field. This coupling inherently provides the advantages of both system and 
thus providing a versatile platform of electrical control in droplet generation in microfluidic devices.

\subsubsection{Material and methods}

The material used in this study is mainly PDMS microfluidic devices fabricated via soft-lithography $[67,68]$. The devices are flushed with Aquapel solutions to ensure uniform wetting properties throughout the channels. The channels remain hydrophobic after the surface treatment. The electrodes are fabricated by melting indium solid solder wire (Indalloy 19, GPS Technologies $\mathrm{GmbH}$ ) into the patterned microchannels [86]. In this way, the shape and locations of the electrodes are fixed and no alignments are needed. The wire melts at about $60^{\circ} \mathrm{C}$ and can be inserted easily when the microfluidic devices are placed on a hotplate. Figure 2.8 shows the schematic sketch of the device.

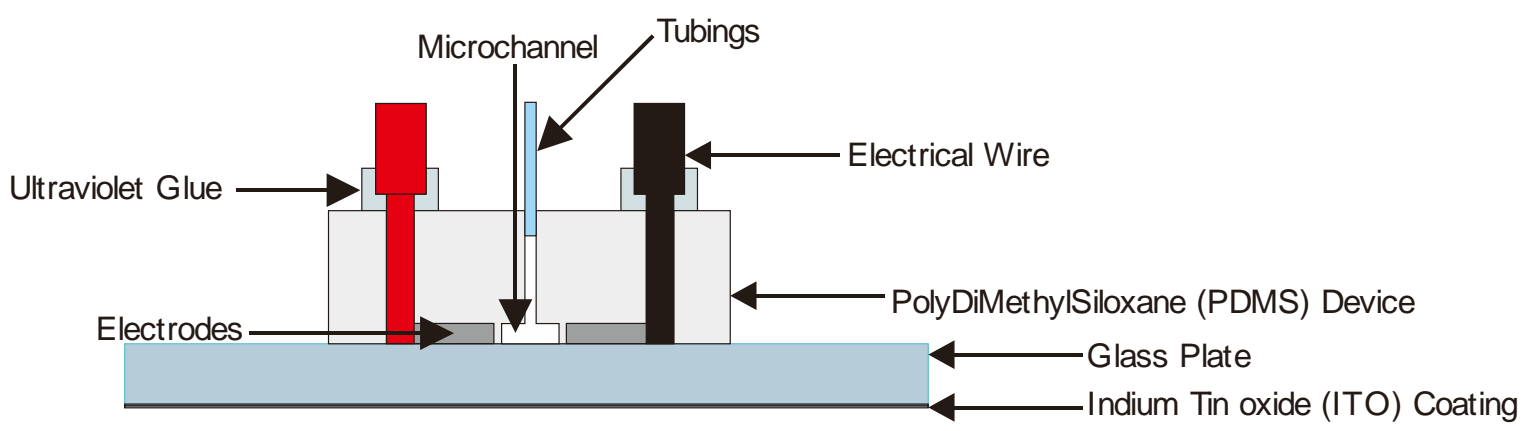

Figure 2.8: Schematic sketch of the microfluidic device. The electrical wires connect the electrodes to the power source and ground. The ultraviolet (UV) glue (3526, Loctite) holds the wires in place to ensure contact with the electrodes at all times. The ITO (CG-60IN-S215, Delta Technologies) acts as an additional electrode which is used mainly to ground the system to prevent stray voltages at the bottom of the device. The wires used are color coded where red represent a connection to the power source and black represent a connection to the ground. 
A flow focusing geometry with and without the orifice (Figure 2.9 and 2.10) is used to investigate the dependence of the size of the droplet produced with an applied AC voltage. Water-in-oil (W/O) droplets are first produced by flowing milli-Q water in the middle of the channel as the dispersed phase fluid. Mineral oil (M5904, Sigma Aldrich) with $5 \% \mathrm{wt} / \mathrm{wt}$ of non-ionic surfactant (SPAN 80, Sigma Aldrich) flows in the two side channels as the continuous phase fluid.

(a)
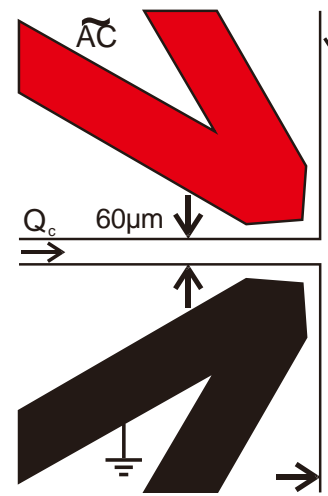
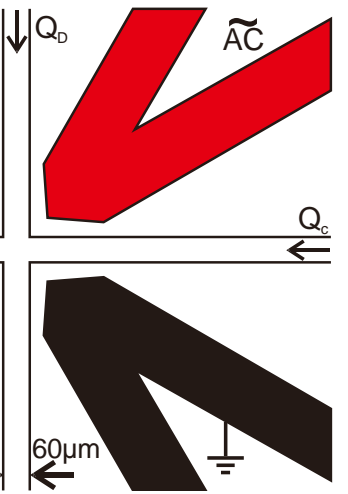

(b)

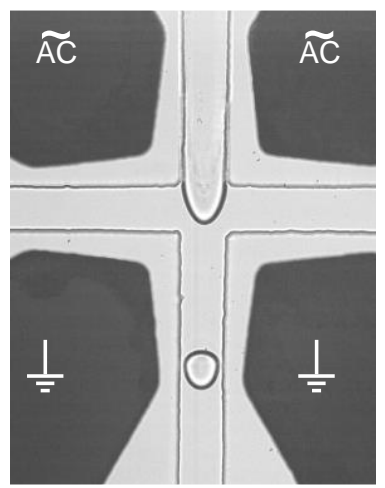

Figure 2.9: Flow focusing device with straight channels. (a) Schematic sketch and (b) Picture of droplets produced in the channels. The depth of the channel is about $35 \mu \mathrm{m}$. The separation distance between the top and bottom pair of electrodes is about $100 \mu \mathrm{m}$.
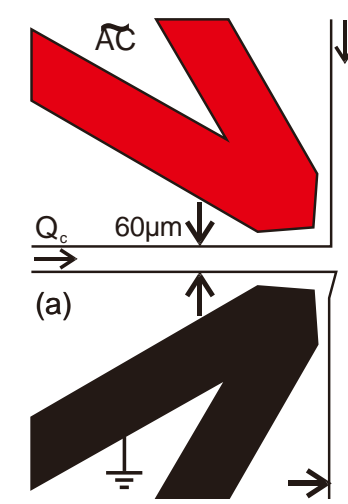
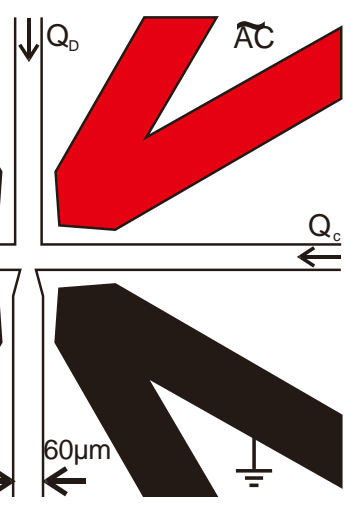

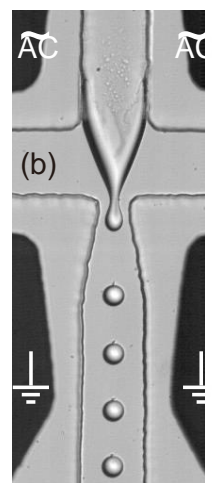

Figure 2.9: Flow focusing device with a $20 \mu \mathrm{m}$ orifice. (a) Schematic sketch and (b) Picture of droplets produced in the channels. The dimensions and height are the same as above. 
A function generator (33210A, Agilent) is used to generate sinusoidal signals at various voltages and frequencies. This signal is then amplified using a high voltage power amplifier (623B, Trek). An oscilloscope is also connected to the output of the amplifier to measure the resulting amplified signal. Figure 2.11 illustrates the experimental setup.

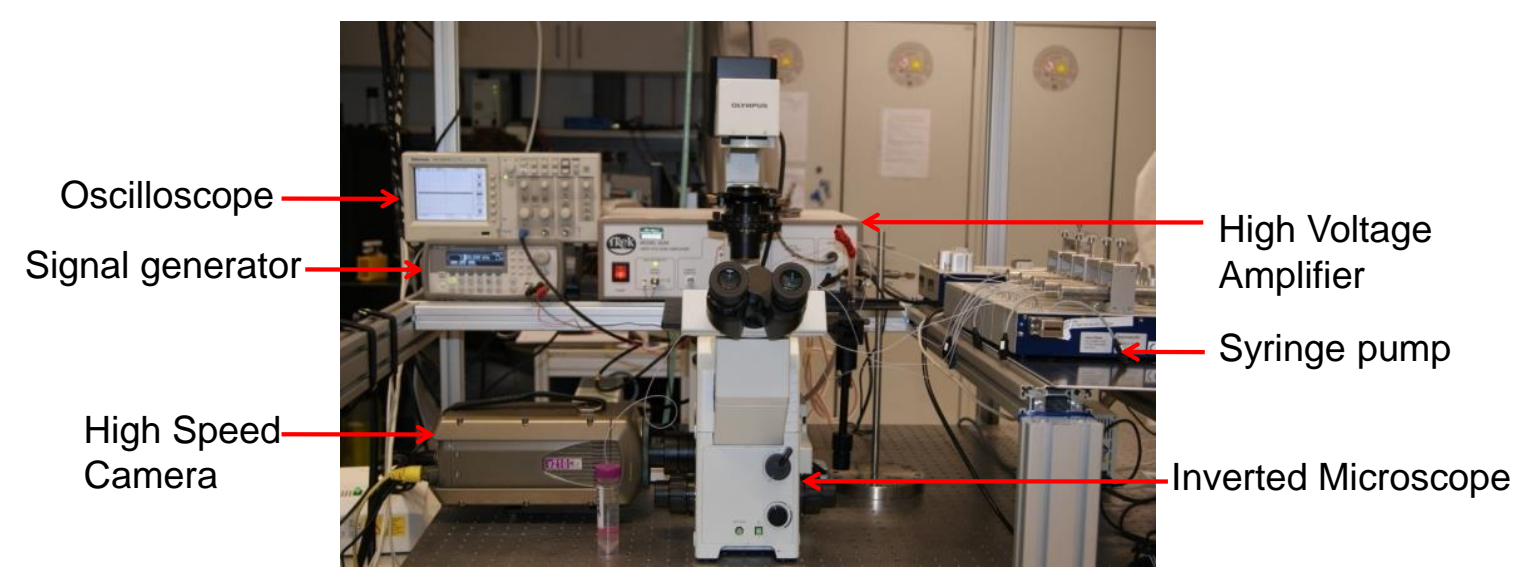

Figure 2.11: Experimental setup illustrating the equipment used in the investigation.

In order to quantify the effect of the $A C$ voltage induced electric field, stable production of W/O droplets are first formed at fixed volumetric flow rates using syringe pumps (neMESYS, Cetoni). The applied AC voltages at different frequencies and applied volumetric flow rates are then varied systematically. Videos of each variation are captured and analyzed using an automated customized software (Matlab, Mathworks) to measure the diameters of the droplets. In the software, the droplet interfaces are detected and the resulting areas are obtained by filling up the interfaces. The diameters of the droplets are calculated by approximating the area as a cylindrical shape. The software also calculates various other parameters such as the droplet formation frequency and droplet velocity. 


\subsubsection{Influence of applied voltage}

The experiments were carried out by increasing the applied voltage from $0 \mathrm{~V}$ to about $1 \mathrm{kV}$ at fixed intervals. The sinusoidal signal is fixed at a frequency of about 50 $\mathrm{kHz}$. The top pair of electrodes is applied with the AC voltage and the bottom pair of electrodes is grounded together with the ITO glass. The two cases of with and without an orifice are tested with three different sets of flow rates. The flow rate ratio $\varphi\left(Q_{d} / Q_{d}\right)$ is fixed at ten for a qualitative comparison.

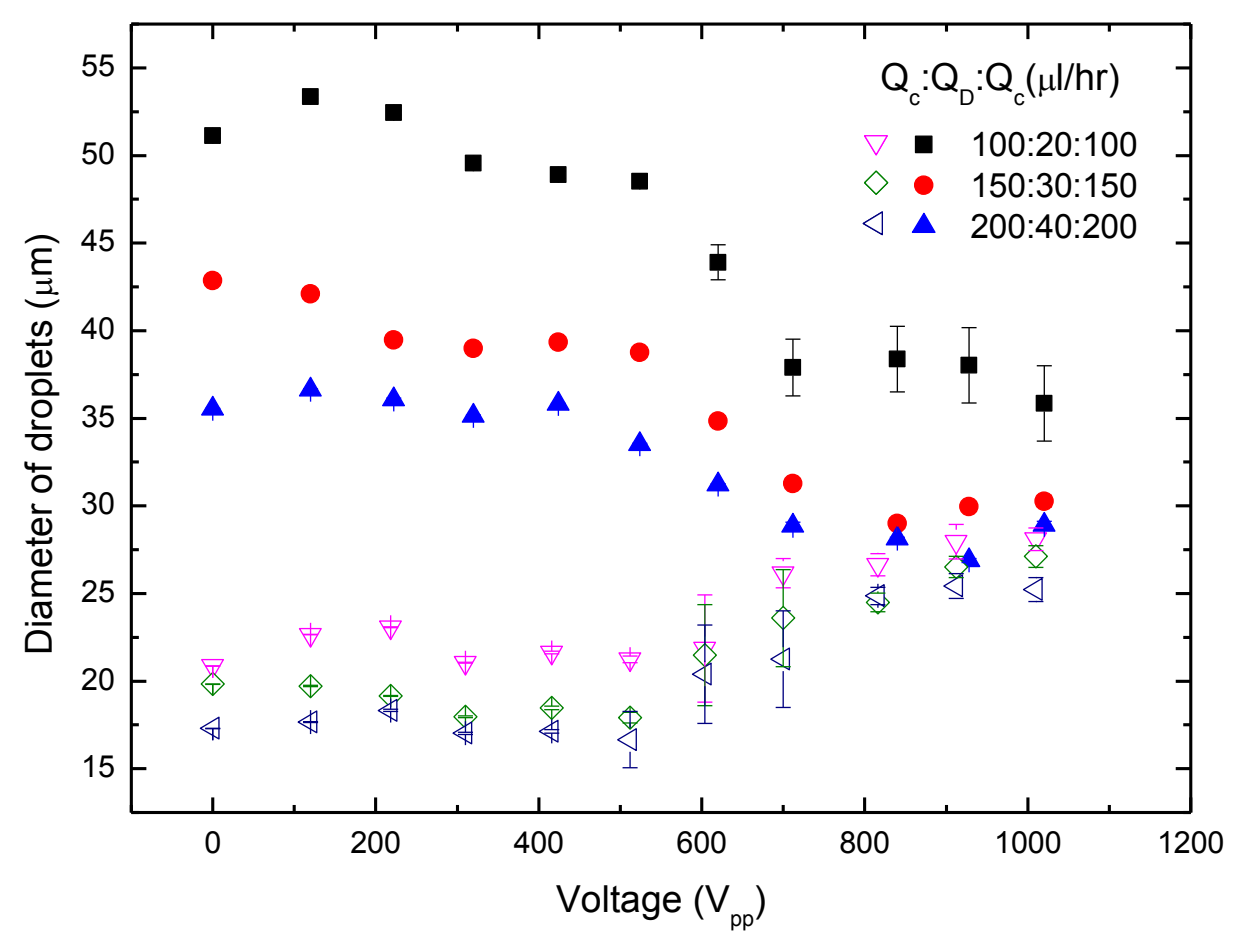

Figure 2.12: Influence of applied voltages at a fixed frequency of $50 \mathrm{kHz}$. Three sets of flow rates are tested. The solid symbols represent results for the case of a straight channel. The open symbols represent results for the case with an orifice of $20 \mu \mathrm{m}$. Both channel widths are $60 \mu \mathrm{m}$ and channel height of about $35 \mu \mathrm{m}$. The volumetric flow rates and ratio are fixed for both cases for comparison. 


\section{Geometry A: Straight channel Geometry B: $20 \mu$ m orifice}
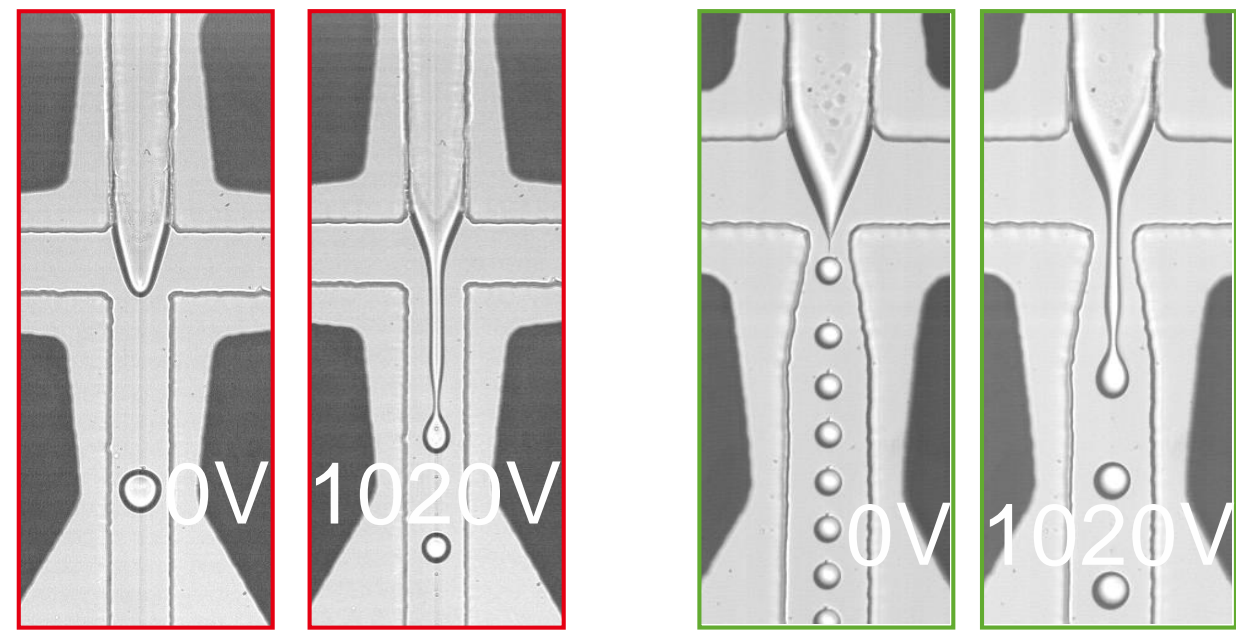

$Q_{C}: Q_{D}: Q_{C}(\mu l / h r)=150: 30: 150$ for both cases.

Figure 2.13: Snapshots of droplets formed at different applied voltage. (a) Straight channel and (b) with a $20 \mu \mathrm{m}$ orifice. The snapshots are taken with different objectives of different magnification.

Indeed, the electric field induced by the AC voltage results in a change in the size of the $\mathrm{W} / \mathrm{O}$ droplets formed in both geometry as displayed in figure 2.12 and 2.13. In the case of the straight channel, the diameter of the droplets decreases for all the three sets of given flow rates. In the case of with the orifice, the diameter of the droplets increases slightly for all the three sets of given flow rates. In both cases, the droplets first formed in the dripping regime and give way to the jetting regime at about $600 \mathrm{~V}_{\mathrm{pp}}$.

\subsubsection{Influence of applied frequency}

In the following, we fixed the volumetric flow rate at $Q_{d}=20 \mu \mathrm{l} / \mathrm{hr}$ and $Q_{c}=100 \mu \mathrm{l} / \mathrm{hr}$ for each side of the continuous phase inlet. Similar experimental tests with voltage variations similar as the previous are carried out. However, the sinusoidal signal 
frequencies are varied between 50 to $10 \mathrm{kHz}$ to investigate the influence of the applied frequencies. Figure 2.14 shows the experimental results obtained.

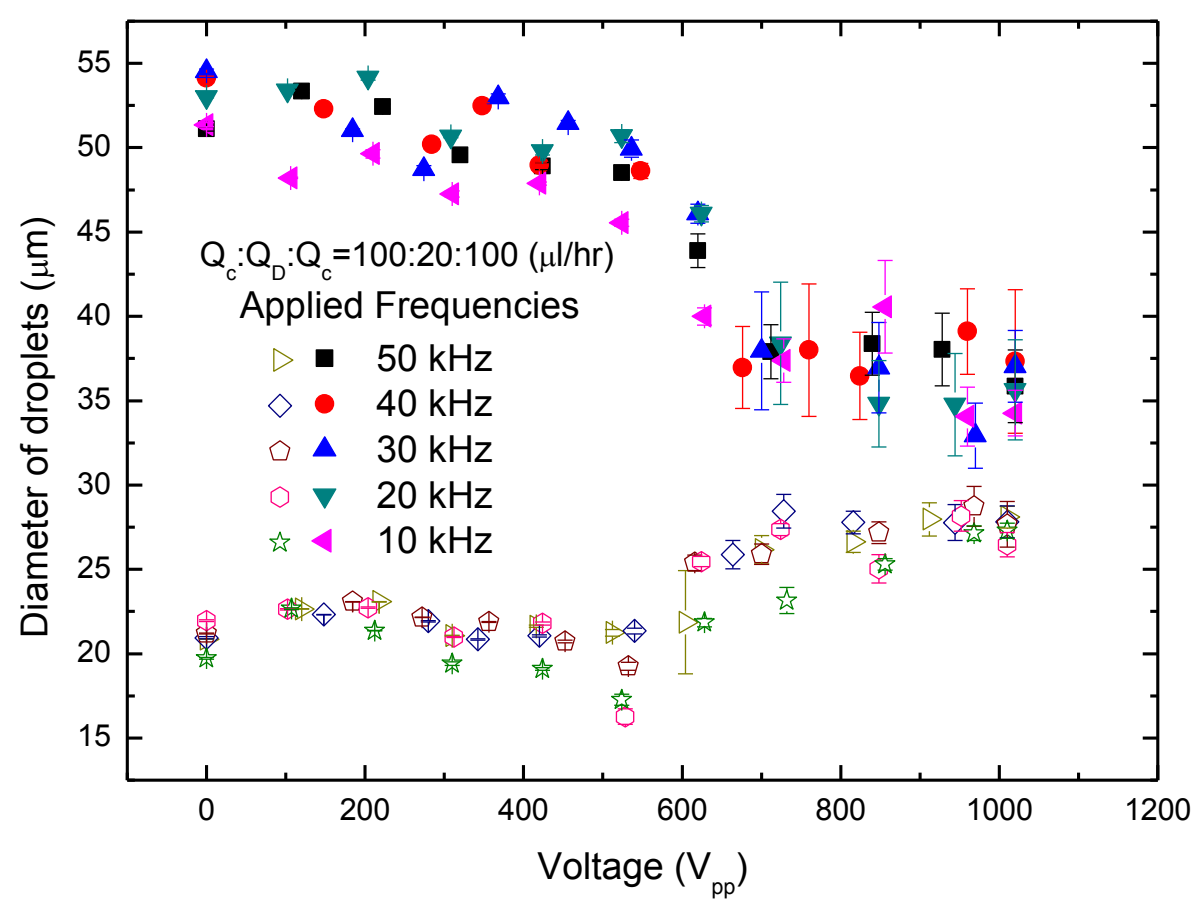

Figure 2.14: Influence of applied frequencies. The solid symbols represent results for the case of a straight channel. The open symbols represent results for the case with an orifice of $20 \mu \mathrm{m}$.

Experimental results obtained at different applied frequencies show negligible differences in the measured droplet diameters when the applied voltage increases from 0 to about $1 \mathrm{kV}$ for both cases. This is because the applied frequencies are much higher than the droplet production frequency. The droplet formation frequencies is in the order of a few hundreds hertz while the frequencies of the signal is at a factor of at least 100 times higher. Moreover, the interplay between conductivity of the fluid and the applied frequencies are not investigated. Only the case with milli-pore water used as the dispersed phase fluid is tested. More detailed experimental investigations with fluids of different conductivities at different applied frequencies are needed to elucidate the behavior. 


\subsection{Discussion}

\subsubsection{Geometrical alteration}

Geometrical factors plays an important role in controlling the formation of monodispersed droplets in microfluidic devices [87]. Hence in order to exploit the observed phenomenon, several alterations to the current designs are needed. The first geometrical alteration is the increase of the width of the microfluidic channels from the initial $60 \mu \mathrm{m}$ to $100 \mu \mathrm{m}$. The channel height is kept constant at $35 \mu \mathrm{m}$ to allow a systematic system. This is because the channel width scales with diameter of the droplets generated [75]. A larger channel width will in turn allow the generation of larger droplets for subsequent electrical manipulation. In the previous experiments, we observed a change in the diameters of droplets formed with the application of an AC voltage induced electric field. This change is relative to the applied voltage. However, we only observed about a maximum of $30 \%$ change in the diameter of the droplets at the maximum applied voltage of about $1 \mathrm{kV}$. The measured diameters at $0 \mathrm{~V}$ is about $51 \mu \mathrm{m}$ and at $1 \mathrm{kV}$ is $35 \mu \mathrm{m}$ (figure 2.12). Although in terms of volume of the droplets, this change is amplified as the diameters of the droplets scales with volume by a cubic factor. However, we anticipate a larger change in the diameter of the droplets when the width of the channel is increased.

The design of the electrodes also affects the stability of the generated droplets. This was evident in the formation of liquids jets which sometimes tends to be attracted to the "sharp" edges located at the downstream channel (Figure 2.15). In order to circumvent this, the designs of the electrodes are amended. The new designs will improve the stability of the jets as the elimination of sharp edges reduces the electric field gradient. The separation of the electrodes with respect to the fluidic channel will also be increase from $20 \mu \mathrm{m}$ to about $35 \mu \mathrm{m}$. This is to reduce and eliminate the problem of the cracks as shown in figure 2.15. The PMDS wall separating the electrodes with the fluidic channels acts as an insulation. The current design often results in a low yield during fabrication as the liquid metal tends to break into the "thin" walls. The improvement will allow an anticipated increase in the overall reliability of the system. 


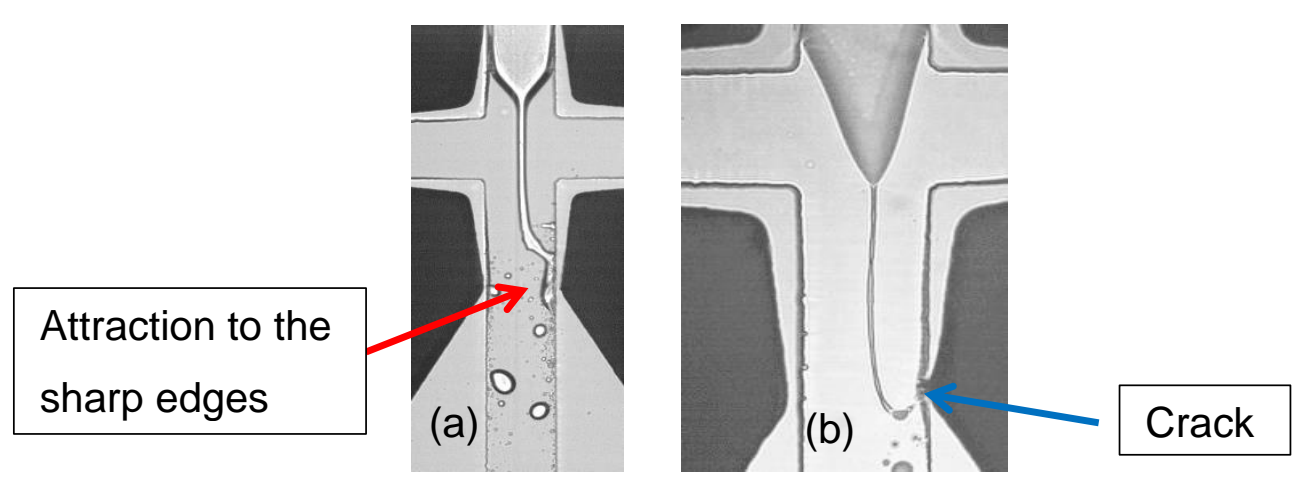

Figure 2.15: Snapshots of the current problems. (a) Attraction to the sharp edges of the electrodes and (b) showing the microcracks.

Figure 2.16 illustrate the simplified new designs which will be used in the next section of the study. In brief, the electrodes are redesigned to resemble a "L" shape and the channel widths are increased to about $100 \mu \mathrm{m}$.

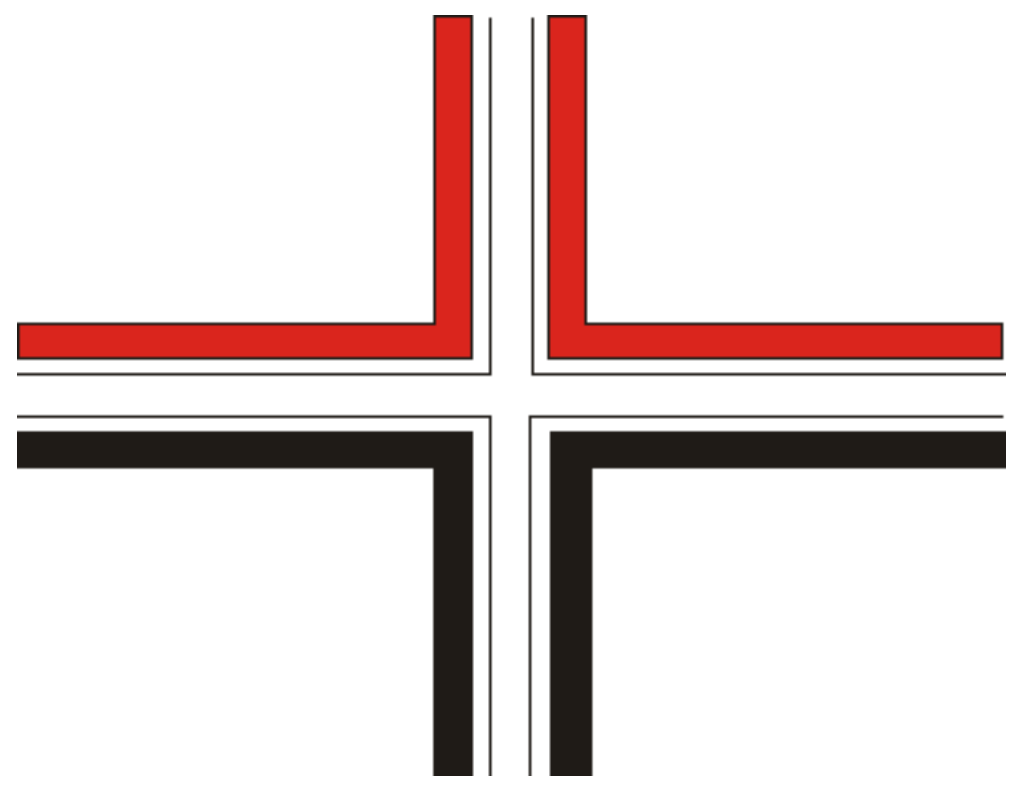

Figure 2.16: Schematic sketch of the simplified design. The channel width is increased to $100 \mu \mathrm{m}$ and the shapes of the electrodes are change to "L" shape. 


\subsubsection{Other factors to be considered}

The current experimental works served only as a conceptual demonstration. A systematic study on various different parameters is needed to understand and explain the observed phenomenon. Physical scaling laws and dimensionless numbers should be developed, calculated and analyze. Beside the applied volumetric flow rates and applied frequencies, other factors should also be considered. For example, the interplay between conductivities of the fluids and the applied frequencies are important electrical parameters for consideration. Figure 2.17 illustrates importance when the conductivity of the fluid is increased by adding small amount of sodium chloride ( $\mathrm{NaCl}$, Sigma Aldrich) salt into the milli-Q water. Unstable droplet production is observed when the applied AC voltage is above 600 $\mathrm{V}_{\mathrm{pp}}$.

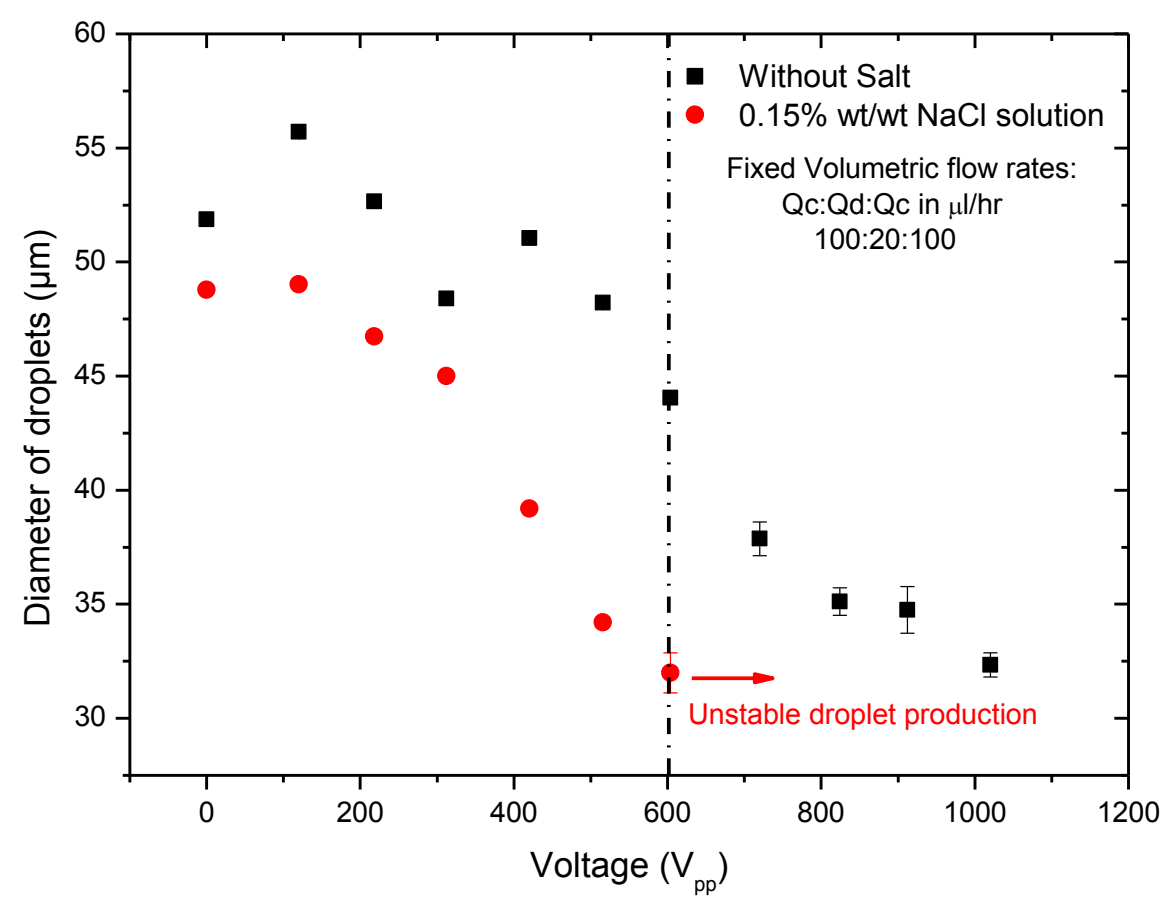

Figure 2.17: Comparison between droplets formed with and without salt. The volumetric flow rates are fixed for both cases. The applied frequencies are also fixed at $50 \mathrm{kHz}$. 
The geometrical configurations of the electrodes also play an important role in the interaction between the induced electric field and the fluid dynamics of droplet formation. In retrospect, the way the electrodes are connected determined the direction of the electric field which in turn affects the droplet formation mechanism. This will need to be studied carefully in the subsequent chapter.

\subsection{Conclusion}

Electroactuation at a microfluidic flow focusing junction offers a new technology to actively control the size of the droplets formed in microfluidic devices [88]. The patented method distinguishes itself from existing electrical control with the use of an AC voltage induced electric field. This method allows the electrodes to be fabricated at ease and also eliminates the need for vexatious alignments. An AC voltage induced electric field is exploited within the junction of droplet formation in a flow focusing configuration. The size of the droplet formed changes with the increase in applied voltage. At different applied frequencies of between 50 to $10 \mathrm{kHz}$, no significant differences are observed in the measured droplet diameters.

Although useful for practical applications, a detailed understanding of the phenomenon requires a simplification of the geometrical parameters of the microfluidic device. In the following, we will show our analysis of the physics of the electroactuation on a simplified geometry. 


\title{
Chapter 3:
}

\section{Microfluidic flow-focusing in ac electric field}

\author{
Say Hwa Tan, Benoit Semin and Jean-Christophe Baret
}

Contents

Chapter 3: Microfluidic flow-focusing in ac electric fields...................................... 38

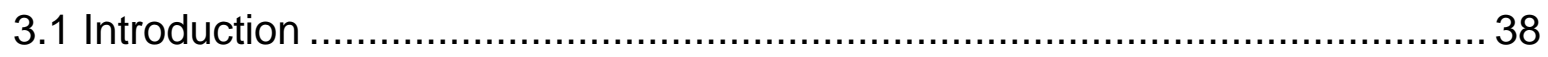

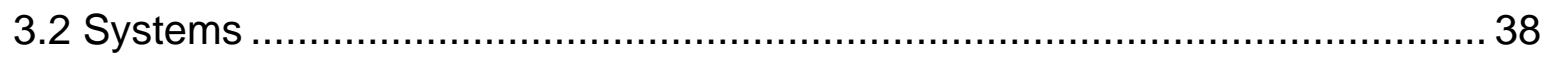

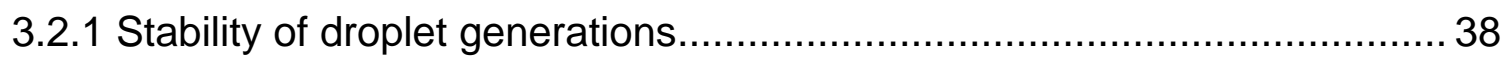

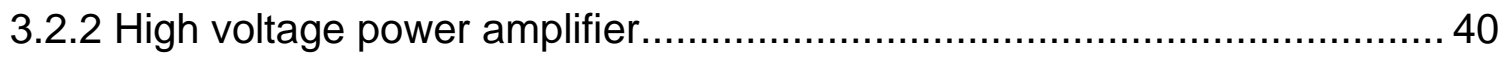

3.2.3 Electrodes configurations …............................................................. 42

3.2.4 Interfacial tension measurements .................................................... 43

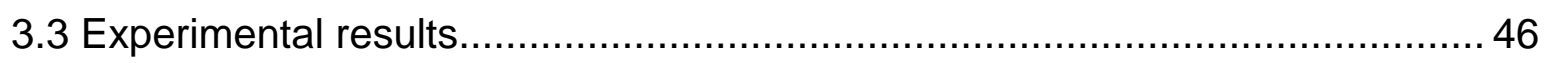

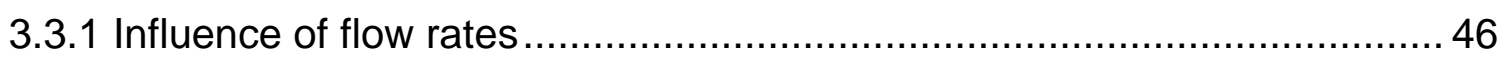

3.3.2 Influence of the conductivities of the dispersed phase fluid .......................50

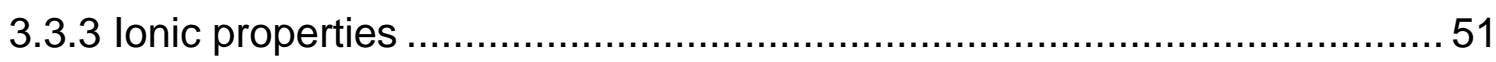

3.3.4 Coupling of frequencies and conductivities............................................ 52

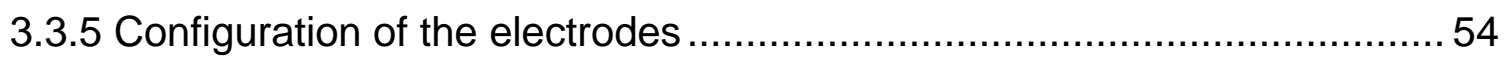

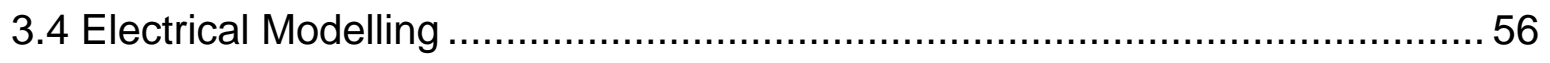

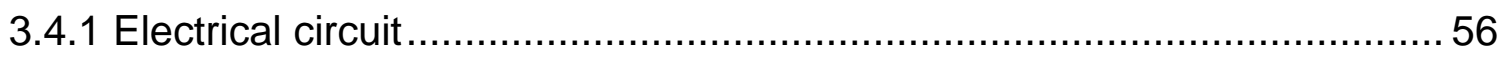

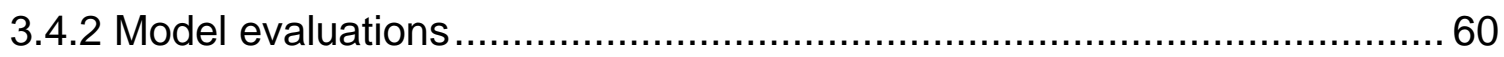

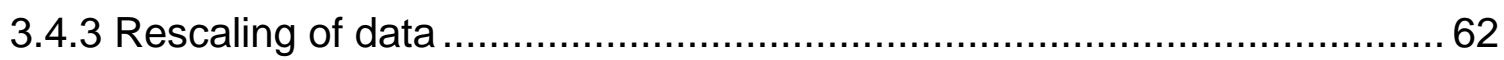

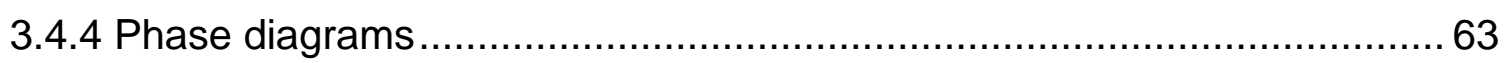

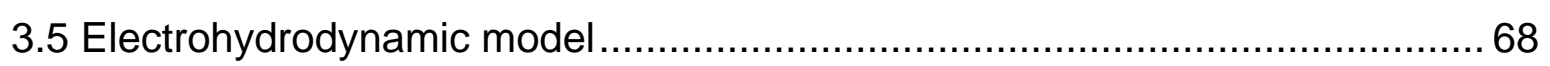

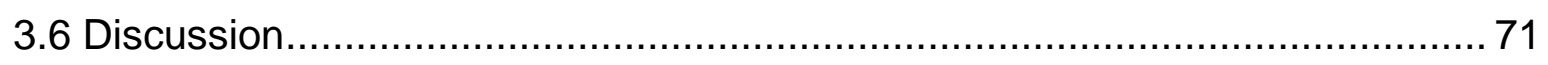

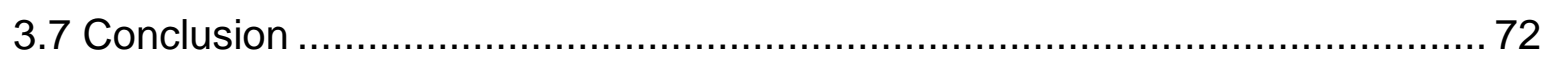




\section{Selected featured back cover page}

Part of this the chapter is described in the following publication:

Microfluidic flow-focusing in ac electric fields [89].

Lab Chip, 2014,14, 1099-1106

DOI: $10.1039 /$ C3LC51143J

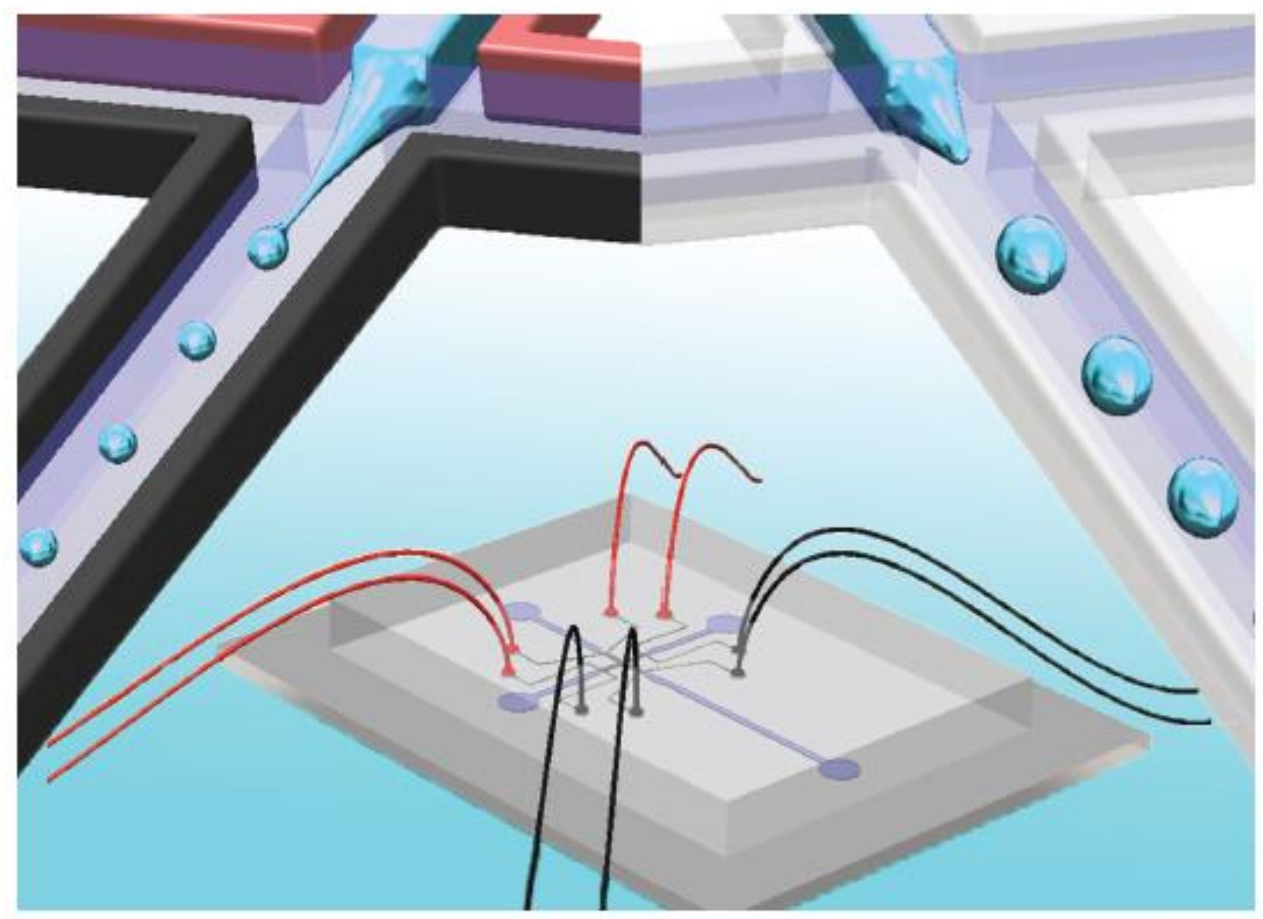

Featuring work from Mr Tan Say Hwa, Dr Benoit Semin and Prof. Jean-Christophe Baret, in the Group Droplets, Membranes and Interface (DMI) at the Max-Planck Institute for Dynamics and Self-organization (MPI-DS), Germany (http://dmi.ds.mpg.de).

Title: Microfluidic flow-focusing in ac electric fields

We demonstrate the control of droplet sizes by an ac voltage applied across microelectrodes patterned around a flow-focusing junction.

As featured in:

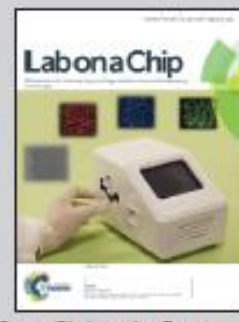

See Jean-Christophe Baret et al, Lab Chip, 2014, 14, 1099.

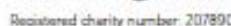




\section{Chapter 3: Microfluidic flow-focusing in ac electric fields}

\subsection{Introduction}

The very first step in a typical droplet-based microfluidic experiments involves the generation of droplets for encapsulation related applications [1, 4, 7, 90-92]. In such applications, it is vital to control the size of the droplets as described in the previous chapters. In this chapter, we will study systematically the proposed method on using an AC voltage induced electric field to form droplets in a microfluidic flow focusing geometry. The electric field is applied directly at the flow focusing junction where water-in-oil (W/O) droplets are formed. Various experiments with different parameters are tested to understand the complex phenomenon. Both the electrical and electrohydrodynamic models are proposed and discussed.

\subsection{Systems}

The proposed emulsification system and quantification method are as per described in the previous chapter. Geometrical alterations to both the channel widths and electrodes geometry are made to improve the reliability and stability of the system. Various characterization tests are also done to ensure the consistency of the results.

\subsubsection{Stability of droplet generations}

The ability to generate droplets of uniform size is of upmost importance in many droplet-based applications [26]. Droplet microfluidics offers a promising route to achieve this requirement due to the precise control of fluid motions in micrometer length scale and laminar flow behavior. The polydispersity in the droplets formed is often in the order of $5 \%$ or less. This is relatively small as compared to different bulk emulsification methods. Several factors that contribute to this are the properties of 
the fabricating material, fluctuation of the syringe pumps and the presence of contaminants. For example, PDMS has poor solvent compatibilities and tends to swell if an inappropriate fluid is used [93]. This will result in huge lateral dimensional changes which may affect the accuracy in droplet generation. Fluctuations induced by the stepper motors in syringe pumps create a pulsating effect which is obvious at low flow rates [94]. Although the use of servo-motor driven syringe pumps (neMESYS, Cetoni) reduces the pulsation significantly, small deviations are still inherent in the system. Last but not least, the presence of contaminants affects the interfacial tension between the fluids. In micro-scale, this difference may be amplified due to the importance of Laplace pressure.

Here we first access the stability and reliability of our system through careful experimental works with the passive system. With a view of quantifying the above, experimental works are devise and carried out. A total of 8 different PDMS microfluidic devices are fabricated using 4 different identical silicon molds. The channel depth is about $35 \mu \mathrm{m}$ and the widths of inlets are about $100 \mu \mathrm{m}$ for all microfluidic devices. Mineral oil (M5904, Sigma Aldrich) with 5\% wt/wt of non-ionic surfactant (SPAN 80, Sigma Aldrich) acts as the continuous phase fluid. Milli-Q water with and without sodium chloride salt act as the dispersed phase fluid in separate experiments. W/O droplets are formed using fixed volumetric flow rate of $Q_{d}=50 \mu \mathrm{l} / \mathrm{hr}$ and $Q_{c}=400 \mu \mathrm{l} / \mathrm{hr}$. After the droplet formation is stabilized, videos of the generated droplets are captured at regular intervals of about 10 minutes continuously for an hour. For each interval, the diameters of about 100 droplets are measured and analyze.

Experimental results show slight variances in the diameters of the droplets (Figure 3.1) over the period of an hour. The average diameter of the droplets obtained is about $82.6 \mu \mathrm{m}$ with a standard deviation of about $1.52 \mu \mathrm{m}$. This corresponds to a coefficient of variance $(\mathrm{CV})$ of about $1.8 \%$. Interestingly, the diameters of the droplets do not vary significantly with the addition of $\mathrm{NaCl}$ salt. Although slight differences are observed in the value of interfacial tension, the minute amount of solute dissolved in the solvent does not affect significantly on the diameters of droplets. This result is useful as it allows a comparison between the different cases in investigating the influence of the conductivities of the dispersed phase fluid. 


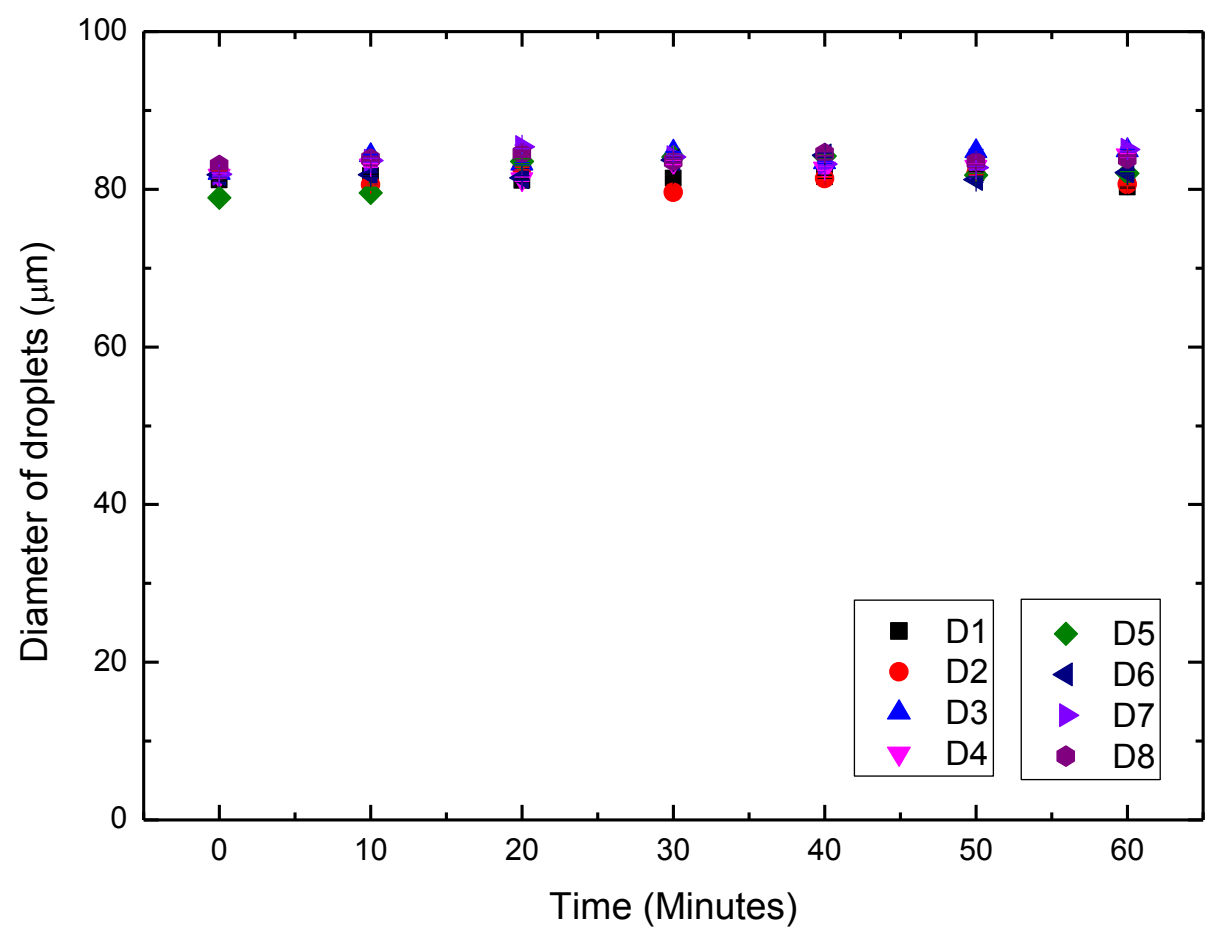

Figure 3.1: Diameters of droplets at different time intervals in a period of 1hour. D1-4 represents the cases of using only milli-Q water. D5-8 represents the cases of using $0.15 \% \mathrm{wt} / \mathrm{wt}$ of $\mathrm{NaCl}$ salt in water.

\subsubsection{High voltage power amplifier}

A high voltage power amplifier (623b, Trek) is used to amplify the input signal generated by the function generator. This amplification allows the generation of electric field strengths which are in the order of Megavolt per meter $(\mathrm{MV} / \mathrm{m})$. This is due to the small separation between the electrodes which is about $170 \mu \mathrm{m}$. However in such amplifiers, the amplification factor or gain is dependent on the performance and the electronic designs of the system. The performance may also deteriorate with time due to aging issue. Limitation or constraints in the bandwidth will also affect the gain at different applied frequencies. Figure 3.2 and 3.3 shows the characterization of the high voltage power amplifier. 


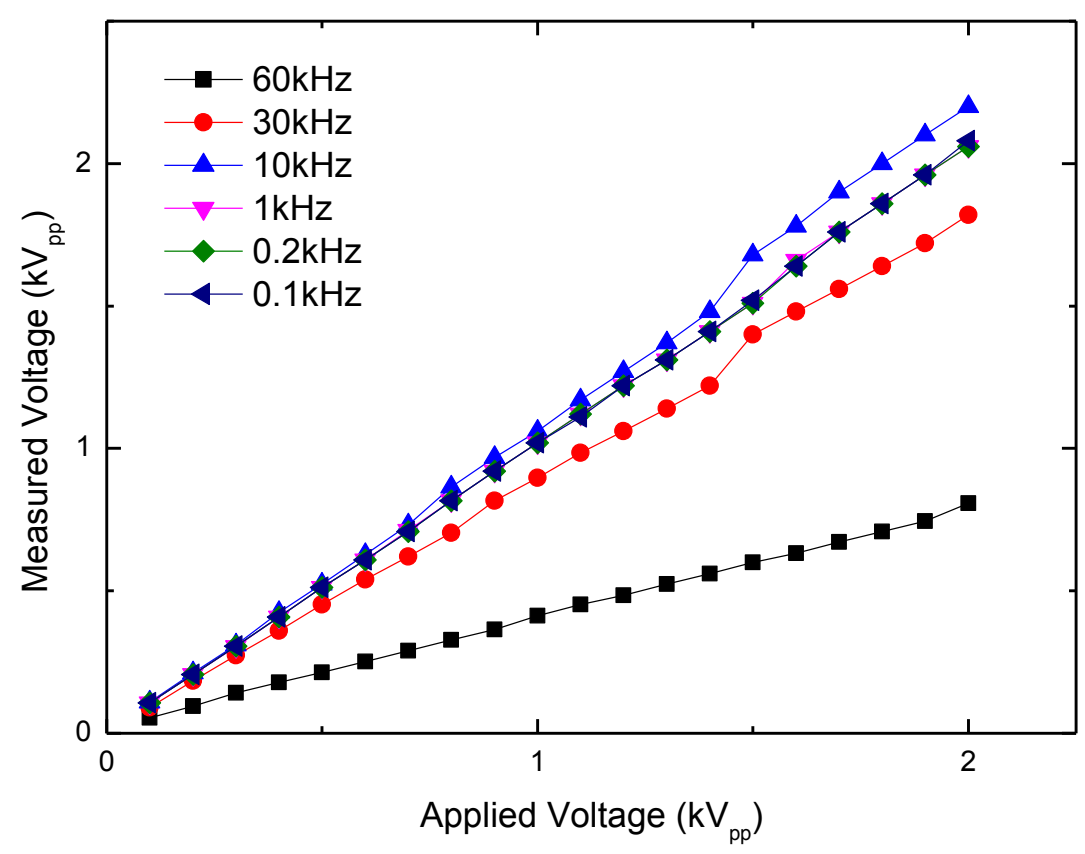

Figure 3.2: Characterization of the amplifier at different applied frequencies. A sinusoidal signal is used for all the cases above.

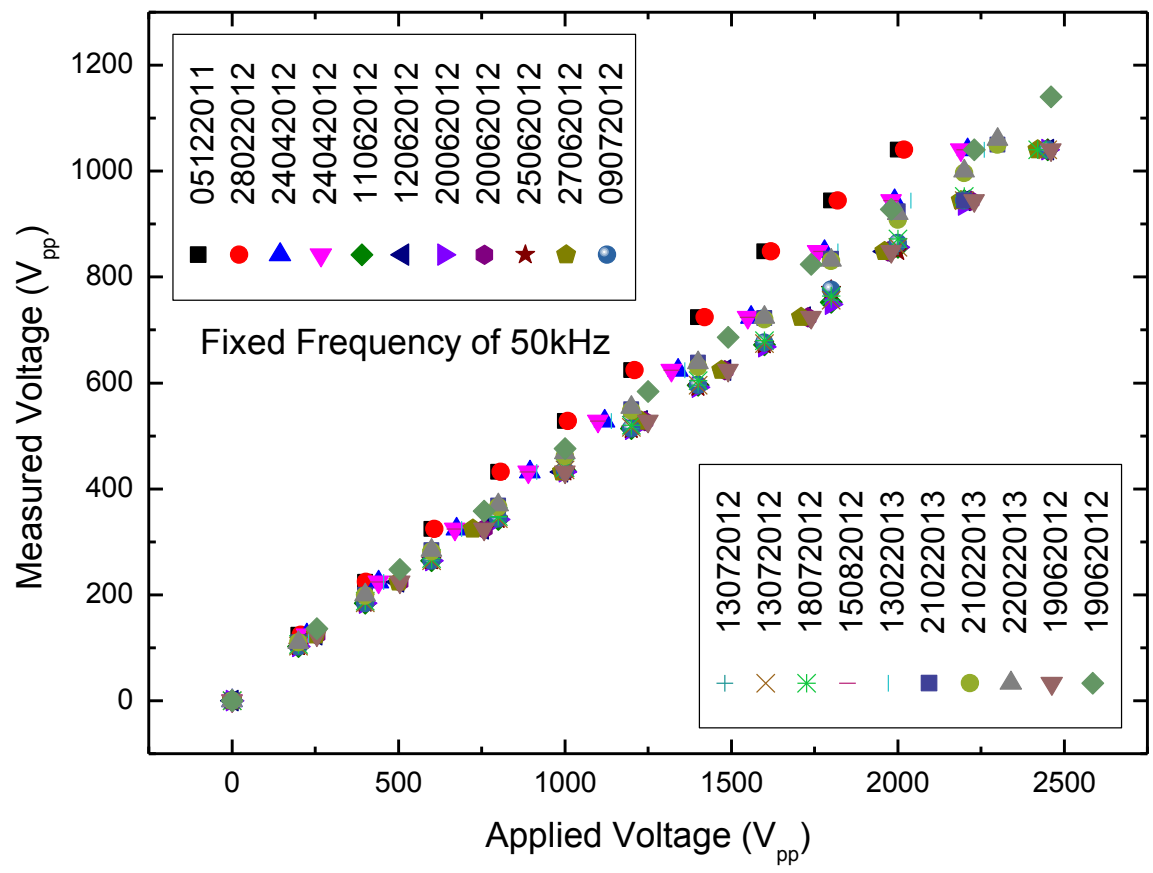

Figure 3.3: Characterization at a fixed frequency. Each symbol represent the dates that reading is taken in the format of DDMMYYY. 
Characterization results show that the gain is dependent on the applied frequencies and changes slightly with aging. Above $10 \mathrm{kHz}$, the gain is less than the specified ratio of 1000 . At a fixed applied frequency of $50 \mathrm{kHz}$, the amplification also changes slightly with aging. Hence, in order to compensate the difference and to ensure that the applied AC voltage is consistent, an oscilloscope is coupled to the output of the amplifier. Meticulous checks on the oscilloscope are made to ensure consistency in the applied voltages during the experiments.

\subsubsection{Electrodes configurations}

The configuration of the electrodes determines the direction of the electric field and how it interacts with the fluids. In the current design, due to the presence of five different electrodes (two in the upstream, two in the downstream and the ITO layer at the bottom of the glass), this results in 32 different electrodes configurations. However, due to the symmetry of the flow focusing geometry, the electrodes have to work in pairs. This reduces the number of configurations by half to 16 different configurations. Experimental trials also show that the system demonstrate the same characteristic when the electrode system is in perfect symmetry. This again reduces the combination by half to 8 different configurations. The two extreme cases where either all the electrodes are applied with or without the AC voltage result in an open circuit which does not generate any electric field. This further reduces the number of configurations to 6 which will be tested in the next section. For simplicity, the table below represents the 6 different configurations which will be investigated in the next section.

\begin{tabular}{|c|c|c|c|c|c|c|}
\hline \multicolumn{7}{|c|}{ 6 different configurations } \\
\hline & $\mathbf{1 : A}$ & $\mathbf{2 :} \mathrm{A}^{\prime}$ & $\mathbf{3 :} \mathrm{B}$ & $\mathbf{4 :} \mathrm{B}^{\prime}$ & $\mathbf{5 :} \mathrm{C}$ & $\mathbf{6 :} \mathrm{C}^{\prime}$ \\
\hline Upstream & AC & G & G & AC & AC & G \\
\hline Downstream & G & AC & AC & G & AC & G \\
\hline ITO & G & AC & G & AC & G & AC \\
\hline
\end{tabular}


Table 3.1: Different possible configurations of connecting the electrodes. AC refers to applying an AC voltage. G refers to the grounding of the electrodes. Upstream refers to the top pair of electrodes, downstream refers to the bottom pair and ITO refers to the conductive oxide layer at the bottom of the glass. $A^{\prime}$ is the configurations where it is the complement of $A$.

\subsubsection{Interfacial tension measurements}

The interfacial tension measurements are carried out using a standard pendent drop method tensiometer (PAT-1M, SINTERFACE). The interfacial tension is measured by fitting the profile of the shape of the droplet to the Young-Laplace equation. In order to obtain accurate measurements in such method, several factors have to noted and considered. Firstly, the measured droplets have to be sufficiently distorted by gravity. This is because interfacial tension is measured by balancing the restoring force of interfacial tension with the gravitation pull due to the weight of the droplet. Several trials and errors have to done before the measurements to determine the optimum size of the droplets that the capillary can withstand. The "wetting" of the metal capillary tube with the fluids will also result in inaccuracy in the measurement. Hence, careful checks and cleaning are made to eliminate the "wetting" effect due to the capillary. Last but not least, the presence of contaminants will also reduce the accuracy of the measurements. To circumvent this, the system is clean and flush several times with ethanol, isopropyl alcohol (IPA) and milli-Q water.

Figure 3.4 to 3.6 depicts the interfacial tension measurements results obtained for

various fluid systems. In the measurements, the diameter of the capillary tube is 2 $\mathrm{mm}$. The densities for the mineral oil and milli-Q water are fixed at $0.84 \mathrm{~g} / \mathrm{cm}^{3}$ and $0.998 \mathrm{~g} / \mathrm{cm}^{3}$ respectively. It is also assumed that the density of both the mineral oil and milli-Q water does not change significantly with addition of small amount of Span 80 surfactant and salts. 


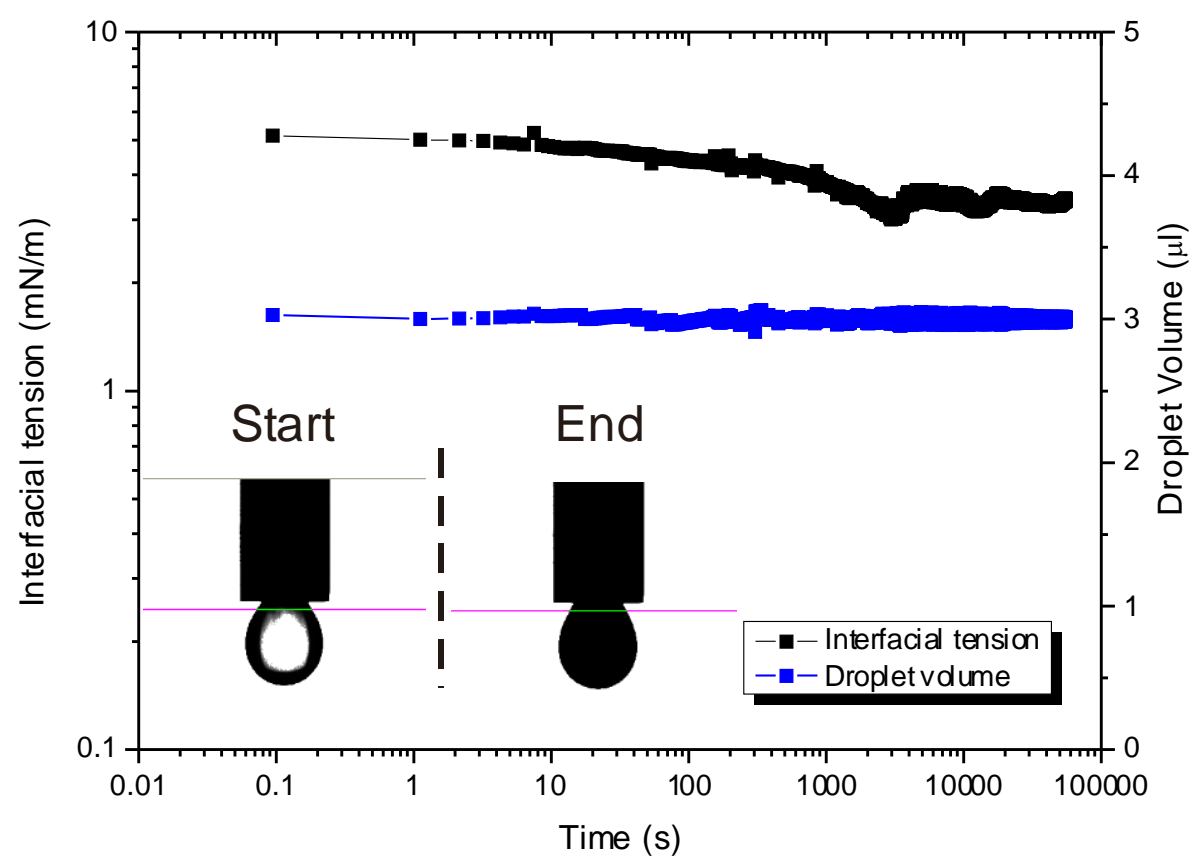

Figure 3.4: Interfacial tension measurement for $5 \% \mathrm{wt} / \mathrm{wt}$ of span 80 in mineral oil with milli-Q water. The insets are the snapshots taken at the start and end of the measurements. The measurements are taken over a period of more than 15 hours.

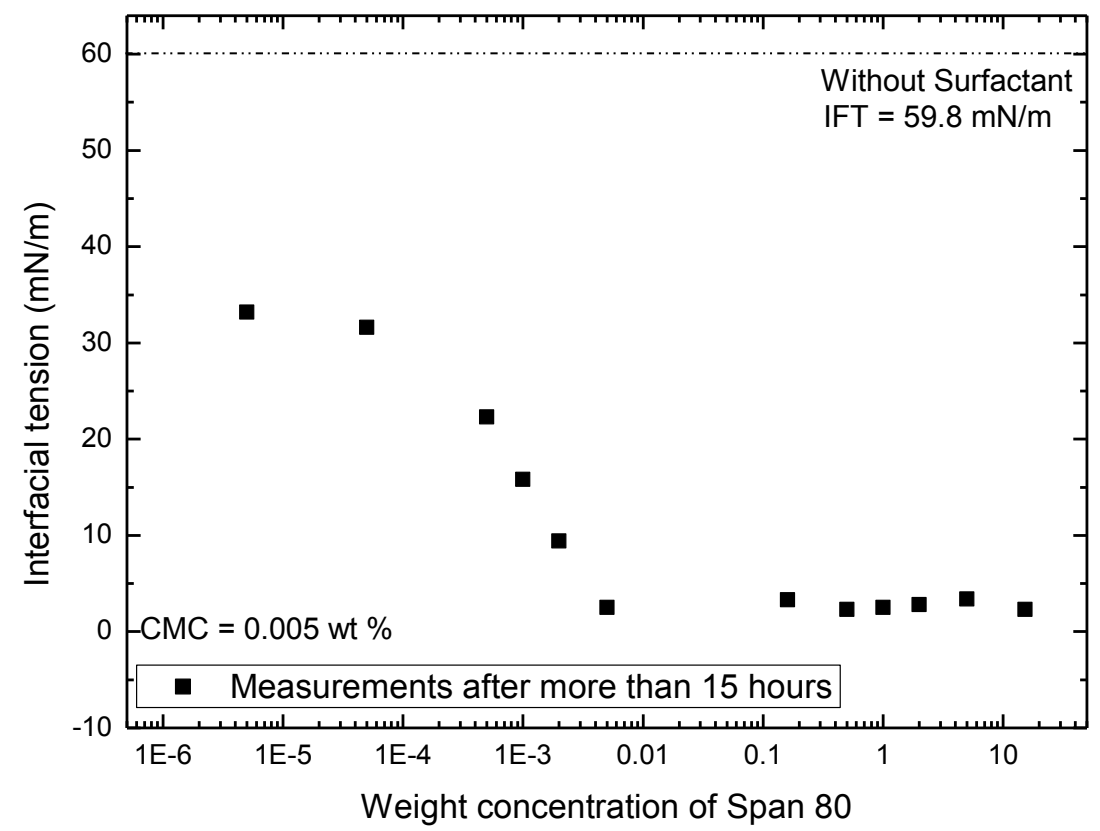

Figure 3.5: Interfacial tension measurements for different concentrations of Span 80. 


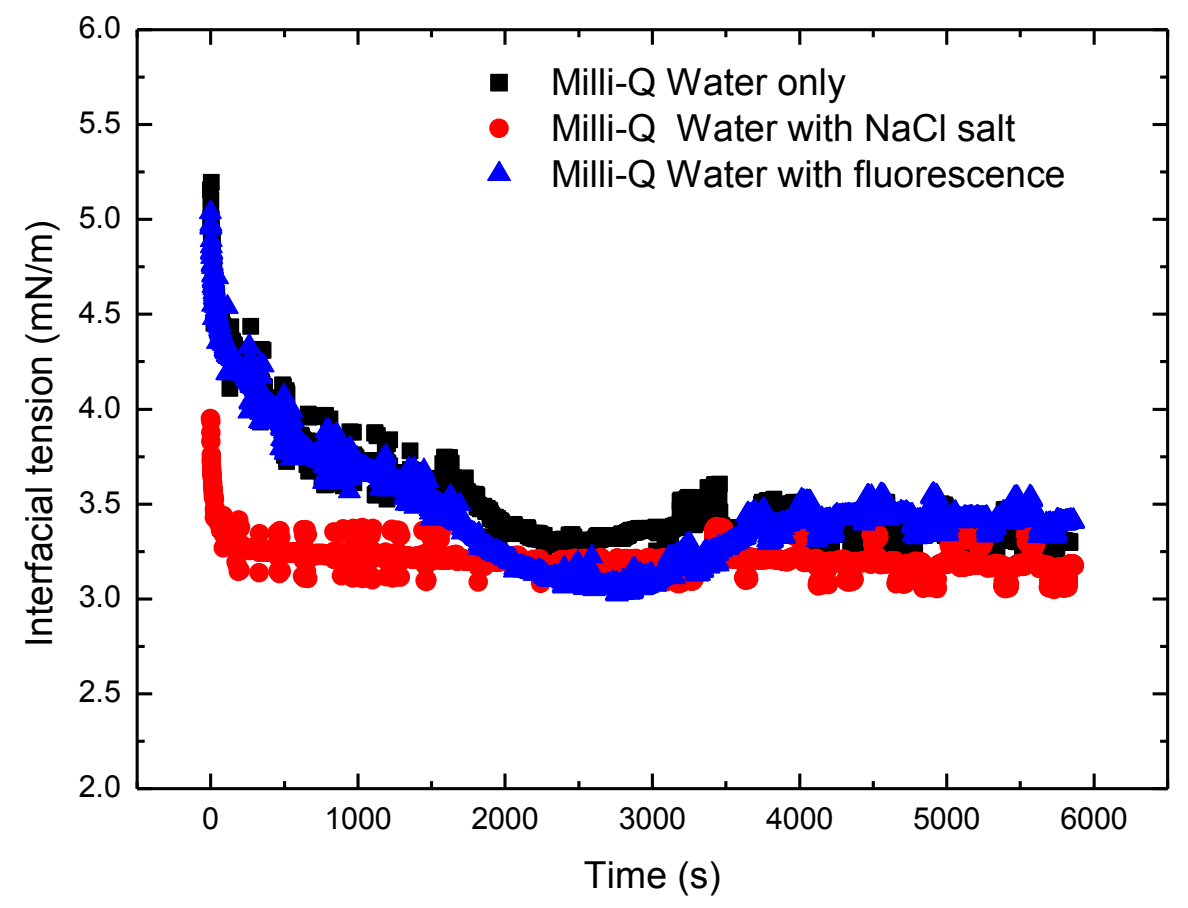

Figure 3.6: Interfacial tension measurements of $5 \% \mathrm{wt} / \mathrm{wt}$ of span 80 in mineral oil with different aqueous solutions.

The interfacial tension measurement characterization reveals several interesting characteristics of the fluid system. For the case of milli-Q water with $5 \% \mathrm{wt} / \mathrm{wt}$ of span 80 in mineral oil, the initial interfacial tension is about $5 \mathrm{mN} / \mathrm{m}$. At equilibrium, the interfacial tension is about $3.5 \mathrm{mN} / \mathrm{m}$ after a period of more than 15 hours of measurements. This result suggests that the absorption kinetics of Span 80 in water is very rapid at the start but it takes a much longer time to reaches equilibrium (Figure 3.4). Figure 3.5 shows the interfacial tension measurements with various concentrations of Span 80 in mineral oil. The result shows that the critical micelle concentration (CMC) is about $0.005 \% \mathrm{wt} / \mathrm{wt}$. In the case of without the addition of surfactant, the interfacial tension between de-ionized water and pure mineral oil is about $60 \mathrm{mN} / \mathrm{m}$. This result also shows that the addition of a small amount of span $80(0.000005 \% \mathrm{wt} / \mathrm{wt})$ will reduce the equilibrium interfacial tension drastically to about $33 \mathrm{mN} / \mathrm{m}$. Figure 3.6 shows the comparison of using different aqueous solutions by adding in small amount of contaminants into milli-Q water such as $\mathrm{NaCl}$ 
salt $(1.5 \% \mathrm{wt} / \mathrm{wt})$ and fluorescence $(10 \mu \mathrm{M})$. The result shows little changes to the equilibrium interfacial tension for the cases tested.

It is also noted that the above measurements may not be an accurate reflection of the true interfacial tension in a microfluidic flow focusing device. This is because of the difference in both the characteristic time scale and volume of the droplets. The typical volume of the droplets formed in the microfluidic flow focusing device is in the order of picolitres but the volume of the droplet measured using the pendent drop is in the range of microliters. The droplet formation frequencies in the microfluidic flow focusing device is in the range of milli-seconds or faster but the pendent drop measures the absorption for a number of hours. However, recent studies reveal that the adsorption kinetics of surfactants is much faster in microfluidic devices when compared to the pendent drop measurements [95]. Hence, the measurements above serve as a good reference for the approximation of the interfacial tension. It is also noted that the absorption kinetics of the surfactants is not within the scope of this study. A detailed explanation of the absorption kinetics of surfactant can be found in this review paper [96].

\subsection{Experimental results}

\subsubsection{Influence of flow rates}

The influence of different volumetric flow rates at different $A C$ voltages is first investigated by using a sinusoidal signal at a fixed frequency of $50 \mathrm{kHz}$. Electrodes in the upstream are applied the AC voltage while the bottom pair and the ITO glass are grounded unless stated otherwise (configuration A). Water-in-oil droplets are generated at fixed volumetric flow rates and flow rate ratio. The dispersed phase fluid is milli-Q water and the continuous phase fluid is mineral oil with $5 \% \mathrm{wt} / \mathrm{wt}$ of span 80 as mentioned in the previous section. The conductivity of the milli-pore water is about $0.3 \mu \mathrm{S} / \mathrm{cm}$. After the droplet generations have stabilized, the applied AC voltage is increase systematically from $0 \mathrm{~V}$ to about $1000 \mathrm{~V}$ at fixed voltage intervals. Videos for each point are then captured and analyzed by measuring at 
least 100 droplets for each point using an automatic image analysis system (Matlab, Mathworks).

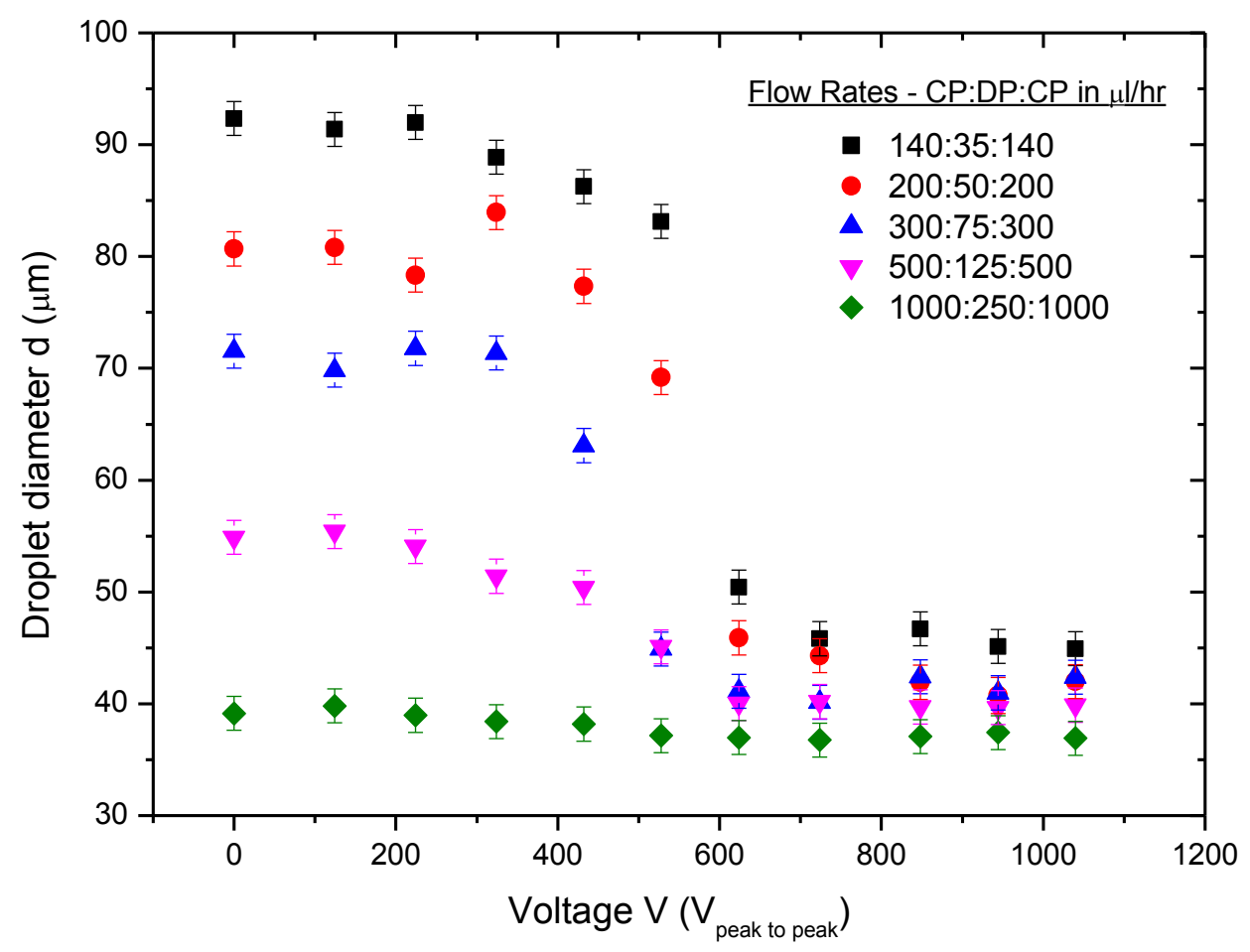

Figure 3.7: Influences of applied AC voltage at different flow rates. A sinusoidal signal of $50 \mathrm{KHz}$ is used here.

Experimental observations show that the diameters of the droplets decreases when the applied AC voltage increases. The decrease in the size of the droplets is accompanied by a change in the droplet formation regime. At low flow rates, the droplets are formed either in the squeezing or dripping regime in the absence of the electric field. Between 600 to $700 \mathrm{~V}_{\mathrm{pp}}$, the droplet formation transit to the jetting regime. However for the case when the droplets are formed in the jetting regime $\left(Q_{c}\right.$ : $Q_{d}: Q_{c}=1000: 250: 1000 \mu \mathrm{l} / \mathrm{hr}$ ), the applied $A C$ voltage does not change significantly the diameters of the droplets. In such a situation, the length of the jet increases dramatically instead with the applied voltages. Figure 3.8 and 3.9 shows the snapshots of the images taken at different flow rates and applied AC voltages. 
Increasing Applied Voltage

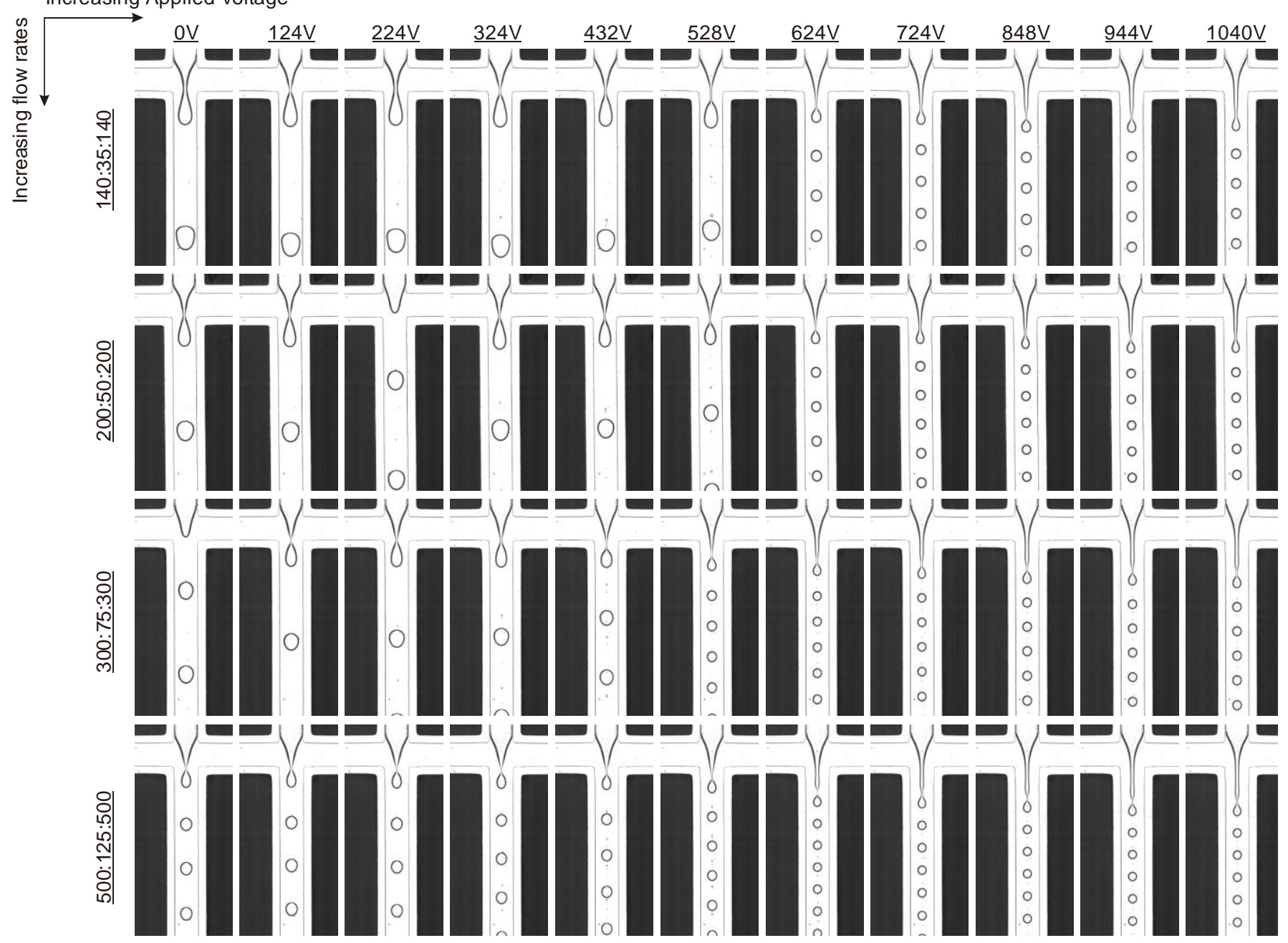

Figure 3.8: Snapshots of the droplets formed at different flow rates and applied AC voltages. 

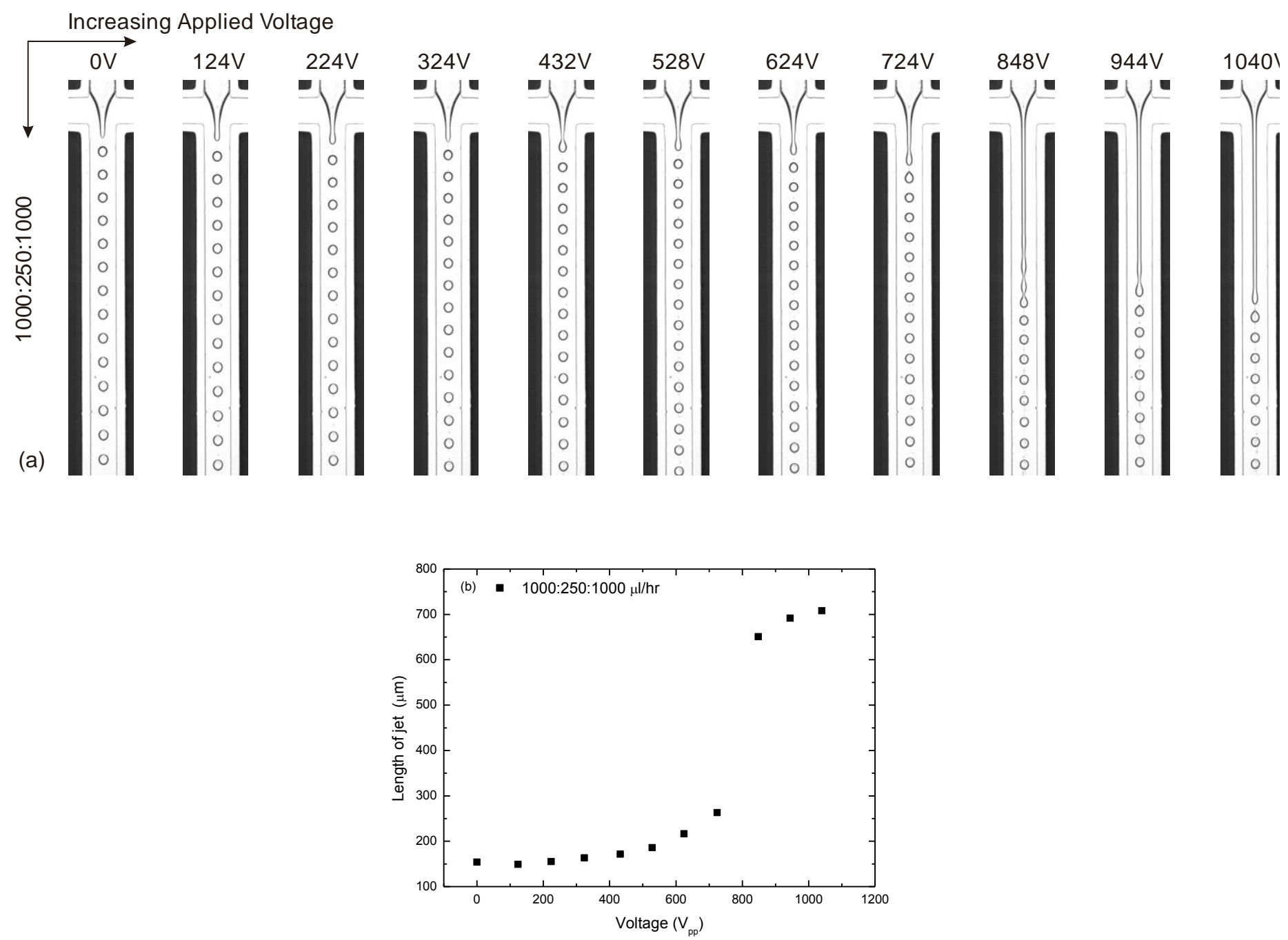

Figure 3.9: (a) Snapshots illustrating the increase in the length of the jet with no significant changes in the size of the droplets and (b) length of the jet as a function of applied voltage. 


\subsubsection{Influence of the conductivities of the dispersed phase fluid}

Next, we investigate the influence of the conductivities of the dispersed phase fluid with the increase in the applied $\mathrm{AC}$ voltage. The electrodes configurations and signal frequency are the same as described in the previous section. A fixed volumetric flow rate of $Q_{c}: Q_{d}: Q_{c}=200: 50: 200 \mu \mathrm{l} / \mathrm{hr}$ was used to generate droplets. The conductivities of the dispersed phase fluid are varied by six orders of magnitude with the addition of small amount of sodium chloride salts. The experimental results are evaluated and shown in figure 3.10.

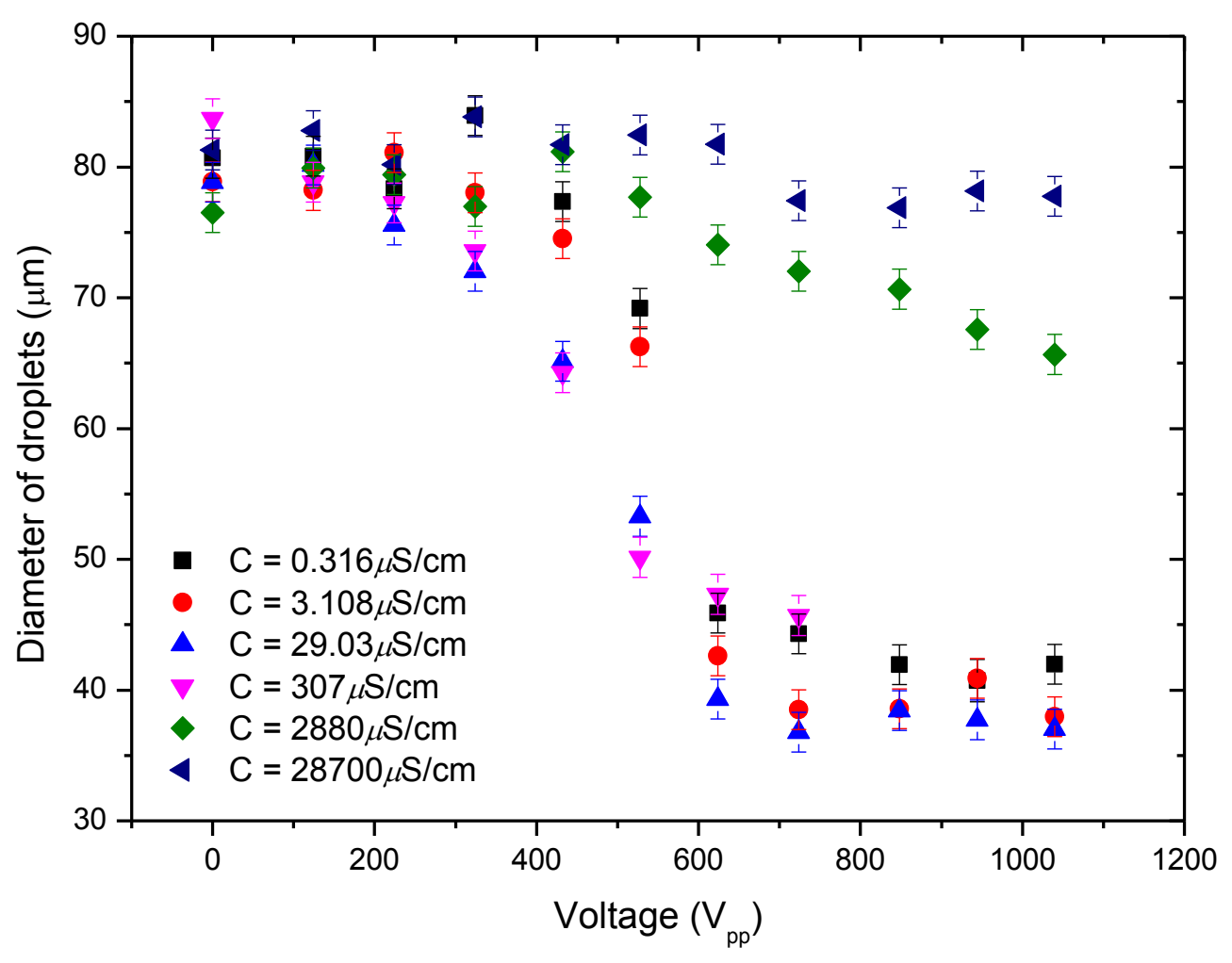

Figure 3.10: Influence of the conductivities of the dispersed phase fluid with the applied AC voltage. The black symbol corresponds to the case of using only milli-Q water without the addition of any sodium chloride. 
Experimental results reveal several interesting observations. In the absence of the electric field (At $V=0$ ), the size of the droplets are similar with only slight variations observed in the diameters. This difference can be attributed to the slight difference in the equilibrium interfacial tension measured previously. Second, when the conductivities of the dispersed phase fluid is increased by 2 order of magnitudes (from about $0.3 \mu \mathrm{S} / \mathrm{cm}$ to about $30 \mu \mathrm{S} / \mathrm{cm}$ ), no significant differences are observed when the applied AC voltage increases. However, when the conductivity is about $300 \mu \mathrm{S} / \mathrm{cm}$, unstable droplet formations are observed beyond the applied voltage of about $700 \mathrm{~V}_{\mathrm{pp}}$. The droplets formed in this case resembled the unstable "whipping" behavior observed in some electrospraying experiments [97]. Last but not least, when the conductivities of the dispersed phase fluid is increased further to about $3000 \mu \mathrm{S} / \mathrm{cm}$ and higher, the diameters of the droplets formed shows only a slight variation despite increasing the voltage to about $1000 \mathrm{~V}_{\mathrm{pp}}$. No changes in the droplet formation regime are observed for both cases. This result clearly shows a strong dependence on the conductivities of the dispersed phase fluid with the applied AC voltage and will be discussed in the later section.

\subsubsection{Ionic properties}

The ionic properties of aqueous solutions are also tested to ascertain the importance of conductivities of the dispersed phase fluid with the applied AC voltage. Aqueous fluids of both sodium chloride $(\mathrm{NaCl})$ and hydrogen chloride $(\mathrm{HCl})$ with similar conductivities are prepared and tested. The electrodes configuration, signal frequency, continuous phase fluids and volumetric flow rates are the same as described previously. The same experimental procedure is performed and the results are quantified as per described in the above. Experimental results shows negligible differences between the two aqueous solutions when the applied AC voltage increases from 0 to about $1000 \mathrm{~V}_{\mathrm{pp}}$. Similar observations are seen as describe previously. This result also eliminates the need to consider the chemical composition of the aqueous solutions and shows that the conductivity of the fluid is the physical quantities governing the change in the size of the droplets formed. 


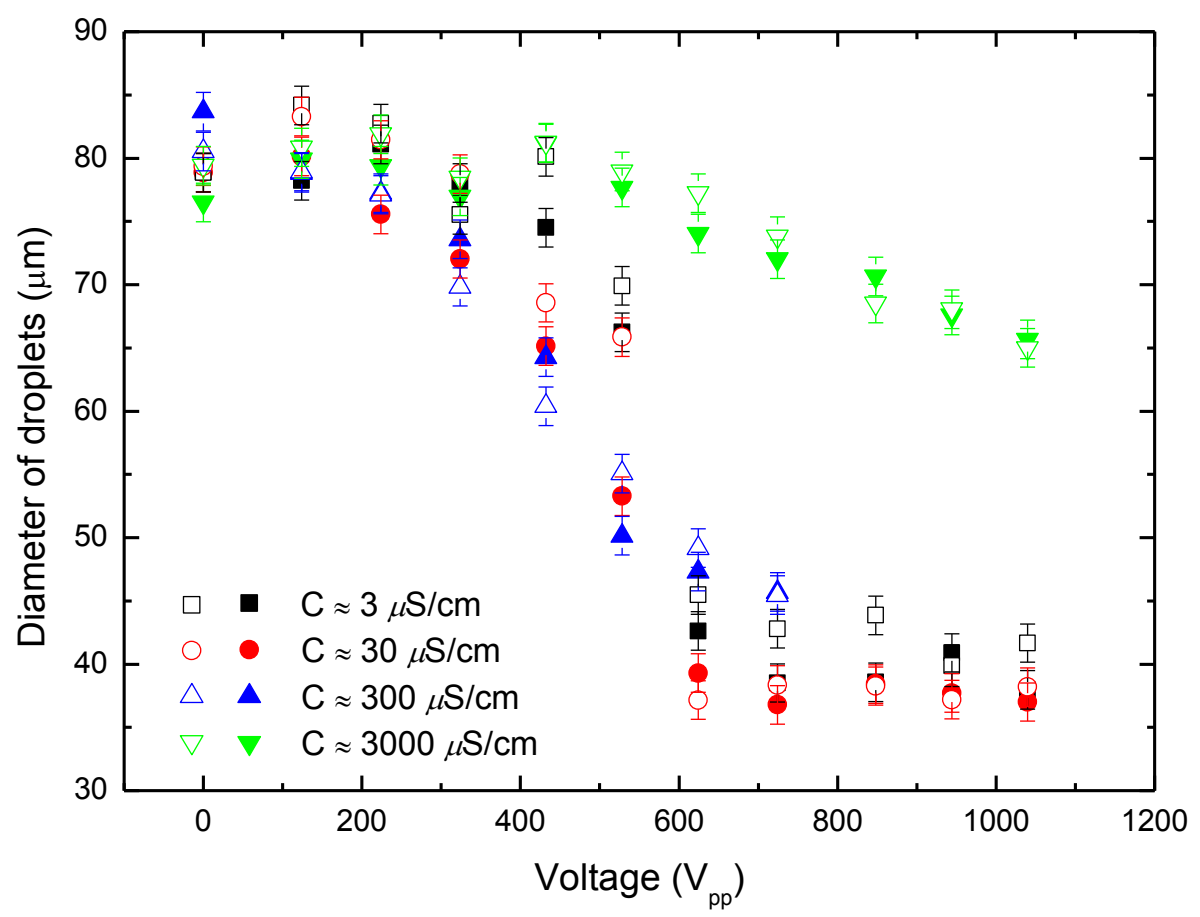

Figure 3.11: Comparison between aqueous solutions of $\mathrm{NaCl}$ and $\mathrm{HCl}$. The symbols in solid represent the cases for $\mathrm{NaCl}$ solutions. The symbols in open represent the cases for $\mathrm{HCl}$ solutions. The shapes represent the different magnitudes of conductivities. A fixed volumetric flow rate of $Q_{c}: Q_{d}: Q_{c}=200: 50: 200 \mu / / h r$ is used.

\subsubsection{Coupling of frequencies and conductivities}

The coupling of both the applied frequencies and conductivities are investigated by varying both the parameters in separate experiments. The electrodes configuration, continuous phase fluid and the volumetric flow rates are as the same as per described previously. Milli- $Q$ water is first tested as the dispersed phase fluid and then replaced by aqueous $\mathrm{NaCl}$ solution of conductivity of about $300 \mu \mathrm{S} / \mathrm{cm}$. The applied frequencies are varied between 50 to $5 \mathrm{kHz}$ and the applied AC voltage is increased from 0 to about $1000 \mathrm{~V}_{\mathrm{pp}}$. Figure 3.12 depicts the effect of applied AC voltages for different frequencies for both the tested fluids with different conductivities. 


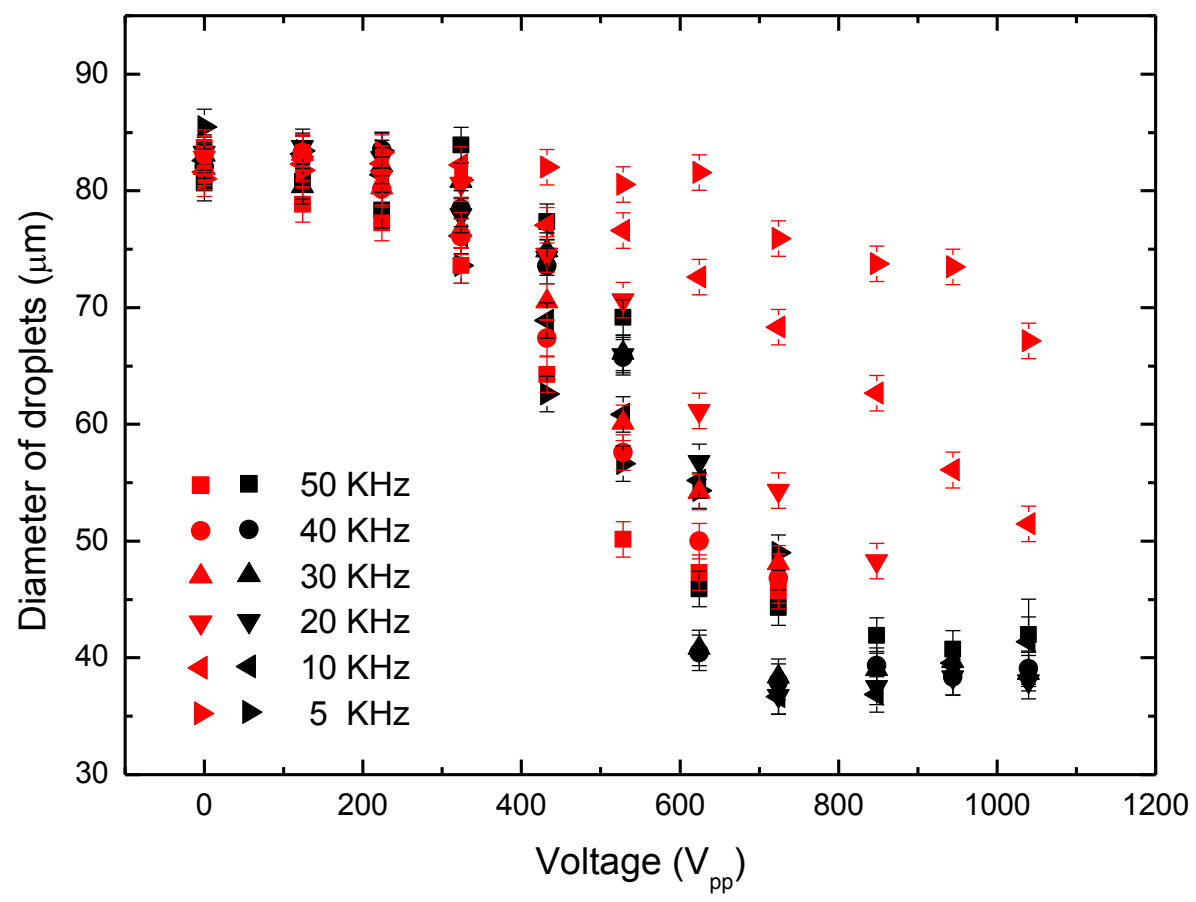

Figure 3.12: Influences of applied frequencies at different AC voltages for solutions with different conductivities. The symbols in black represent the case for milli-Q water and the red symbols represent the case for aqueous $\mathrm{NaCl}$ solution of conductivity of about $300 \mu \mathrm{S} / \mathrm{cm}$ which is about 1000 times higher. The volumetric flow rates are fixed in all the cases at $Q_{c}: Q_{d}: Q_{c}=200: 50: 200 \mu l / h r$.

For the case of using milli-Q water as the dispersed phase fluids, no significant differences are observed when the applied frequencies are varied between 50 to 10 $\mathrm{kHz}$ and when the voltage is increased from 0 to $1000 \mathrm{Vpp}$. This result agrees well with the previous case depicted in chapter 2. However, when the applied frequency reduces to $5 \mathrm{kHz}$, unstable droplet formations are observed for voltages beyond 700 $\mathrm{V}_{\mathrm{pp}}$. For the case of using aqueous $\mathrm{NaCl}$ solution as the dispersed phase fluids, the reduction in the size of the droplets decreases with the decrease in applied frequencies. This result suggested that the interplay between the applied frequencies and conductivities of the dispersed fluids plays a role in determining the change in the size of the droplets with the increase in applied voltage. This will be discussed further in the next section. 


\subsubsection{Configuration of the electrodes}

The configurations of the electrodes determine the direction of the electric field and how the electric field interacts with the fluids. As mentioned previously, a total of 6 different electrodes combinations are considered in this study. In the first case (A), an $A C$ voltage is applied to the upstream pair of electrodes while the downstream pair of electrodes and the ITO are grounded. The second case (B), is when the downstream pair of electrodes are applied with an AC voltage while the upstream pair and the ITO are grounded. The third case (C), is when both the upstream and downstream pairs of electrodes are applied with an AC voltage while the ITO is grounded. The complements of $A, B$ and $C$ refers to cases of the exact opposite in the way the $A C$ voltage is applied.

Experiments for two different set of dispersed phase fluids are conducted for all the 6 different electrodes combinations mentioned. In the first set, milli-Q water is used as the dispersed phase fluid and aqueous $\mathrm{NaCl}$ solution of conductivity of about 3000 $\mu \mathrm{S} / \mathrm{cm}$ is used in the second set. Mineral oil with $5 \% \mathrm{wt} / \mathrm{wt}$ of span 80 is used as the continuous phase fluid for both sets of dispersed phase fluids. The same volumetric flow rates of $Q_{c}: Q_{d}: Q_{c}=200: 50: 200 \mu / / h r$ is used for all the experiments. In all the experiments, the applied frequencies are fixed at $50 \mathrm{kHz}$ and the $\mathrm{AC}$ voltage is increased systematically from 0 to $1000 \mathrm{~V}_{\mathrm{pp}}$. The diameters of the droplets at different applied voltages are measured and plotted in figure 3.13 and 3.14.

Experimental results show similar variations in the diameters of the droplets with the applied AC voltage are observed when the electrodes configurations are in perfect symmetry. This implies that the electrodes combination in A behaves the same as the combination in $A^{\prime}$. For the case of using milli-Q water as the dispersed phase fluid, negligible changes in the diameters of the droplets are observed when the AC voltages increases for both combination $C$ and $C^{\prime}$ (figure 3.13). For the case of using aqueous $\mathrm{NaCl}$ solutions as the dispersed phase fluid, slight differences are observed between the complements for set $A$ and $C$ (figure 3.14). These results ascertain the importance of the ITO which acts as a third set of electrode and has to be taken into account during the modelling of the system. 


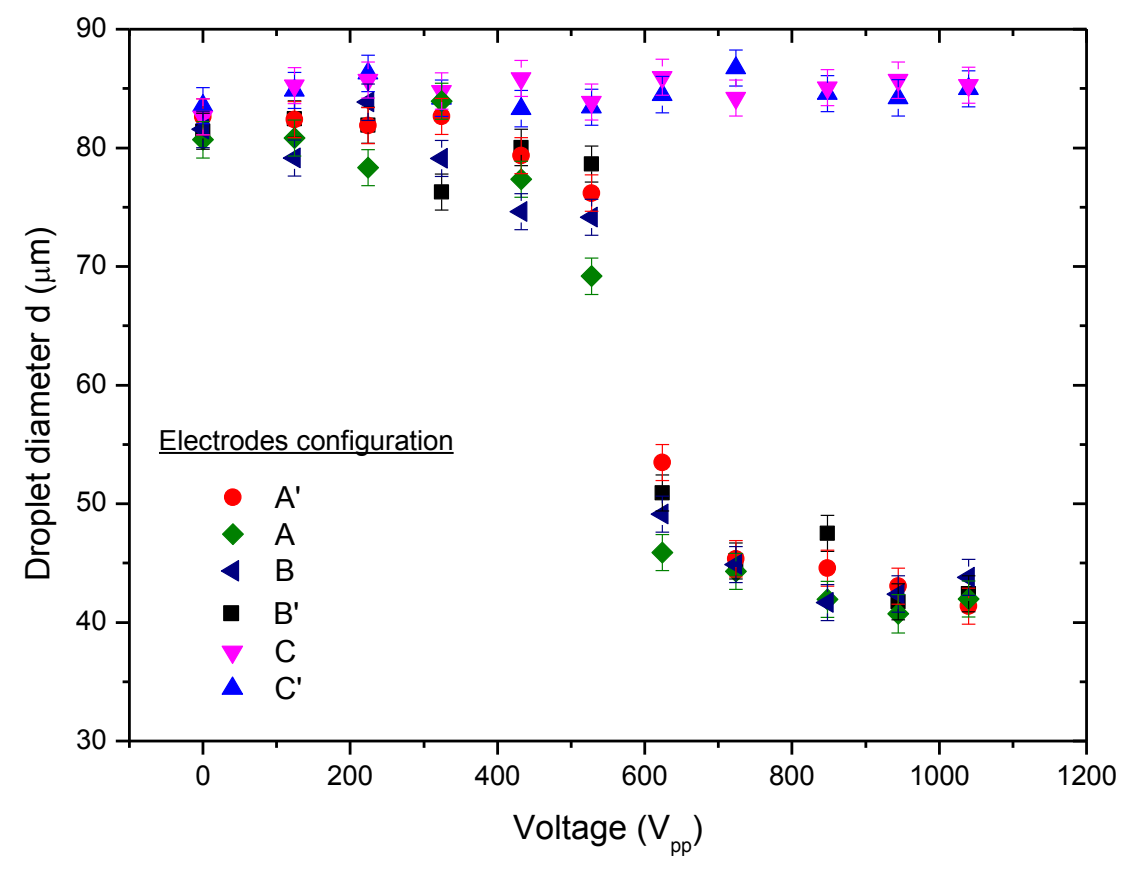

Figure 3.13: Diameters of droplets at different applied voltages in different electrode configurations for the case of milli-Q water.

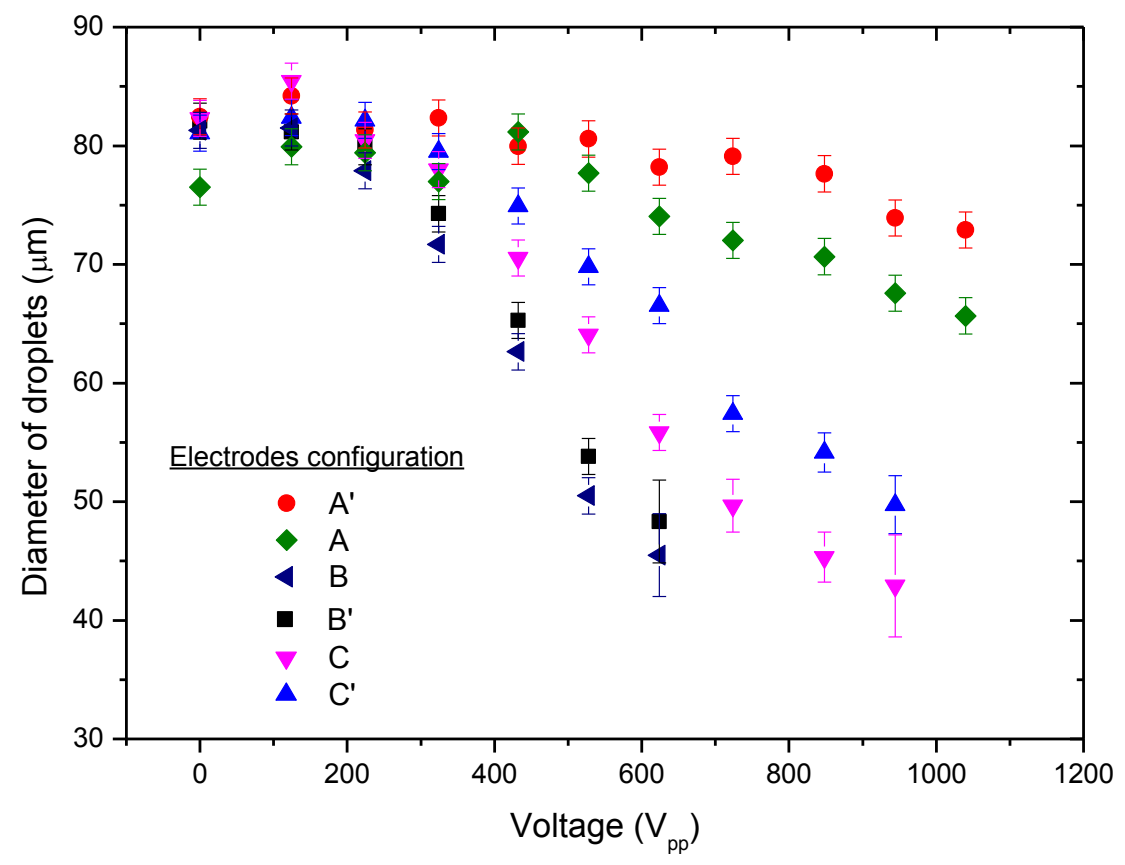

Figure 3.14: Diameters of droplets at different applied voltages in different electrode configurations for the case of aqueous $\mathrm{NaCl}$ solution. 


\subsection{Electrical Modelling}

In this section, we first build an electrical model which can be used as a representation to describe the system. With the simple model, we will address the experimental findings observed in the previous section.

\subsubsection{Electrical circuit}

The experimental findings presented in section 3.3 are intriguing but interesting. The results suggest a strong dependency on both the conductivities of the dispersed phase fluids and the applied frequencies of the sinusoidal signal. Here a simplified electrical circuit model is presented based on the experimental findings observed previously. For simplicity, electrodes in configuration A will be used as the primary basis for the modelling and explanations. Figure 3.15 illustrates the schematic sketch of system and the equivalent electrical circuit.

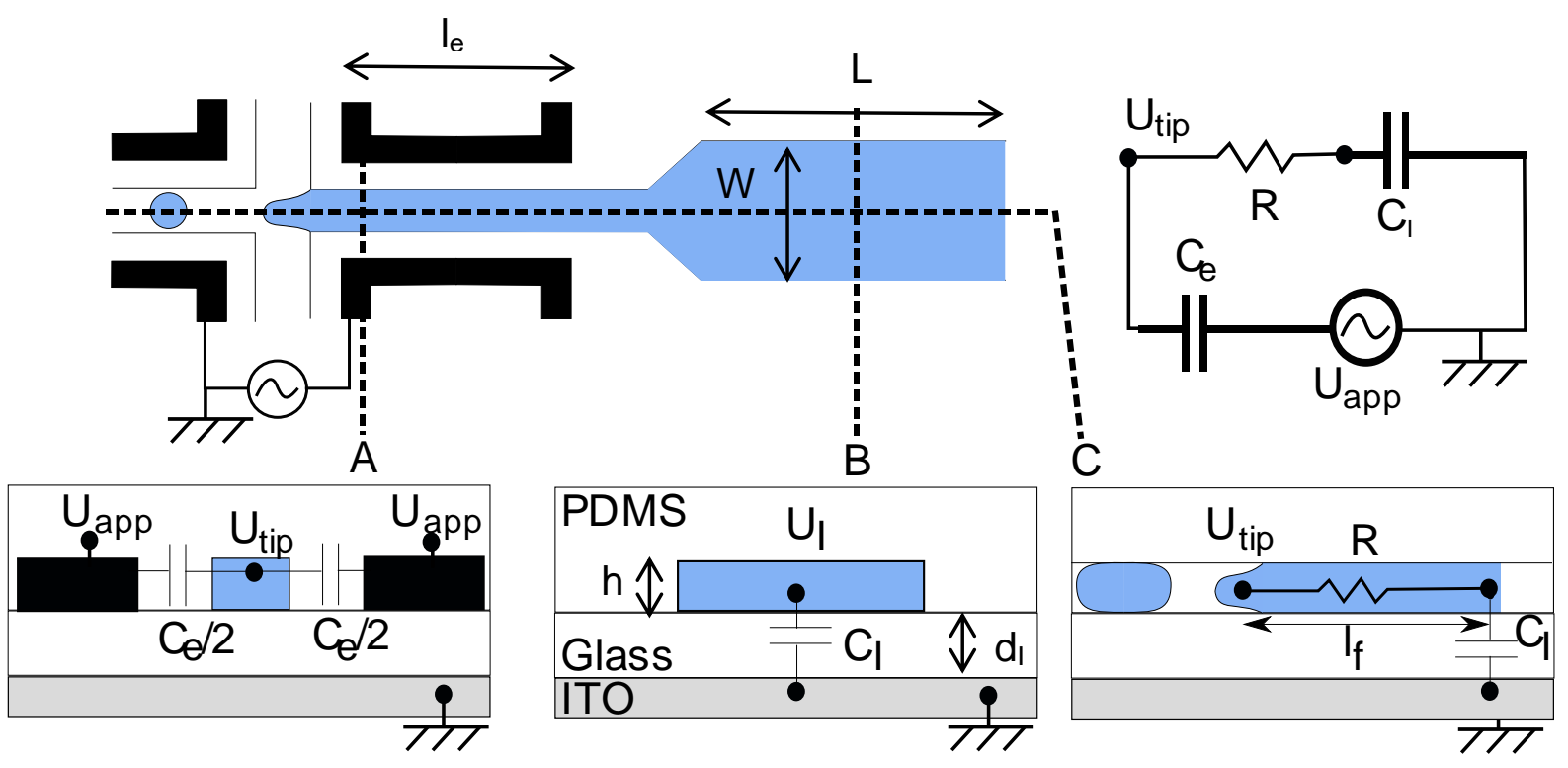

Figure 3.15: Schematic sketch of the microfluidic flow focusing system in configuration $\mathrm{A}$. The sectional cuts represent each part used to derive the equivalent circuit shown. The electrodes are in black and the aqueous phase fluid in blue. 
In electrodes configuration $A$, an $A C$ voltage is applied at the upstream pair of electrodes while the downstream pair and the ITO are grounded. In this case, as the top pair of electrodes is not in contact with the dispersed phase fluid, the PDMS wall which provides the insulation acts as a capacitor $C_{e}$ as shown in the sectional cut $A$. For one side of the electrode, $C_{e / 2}$ can be modelled using the parallel plate approximation as shown in equation (3.1).

$$
C_{\mathrm{e} / 2}=\varepsilon_{o} \varepsilon_{r}\left(\frac{A}{d}\right)=\varepsilon_{o} \varepsilon_{r}\left(\frac{l_{e} \times h}{d_{e}}\right)
$$

Where $\varepsilon_{o}$ is the vacuum permittivity which is about $8.854 \times 10^{-12} \mathrm{~F} / \mathrm{m}, \varepsilon_{r}$ is the relative permittivity of the material which is about 2.5 for PDMS. Here $A$ is the cross sectional area of the electrodes which can be expressed in terms of the length of the electrodes $l_{e}(2200 \mu \mathrm{m})$ multiply by the height of the channel $\mathrm{h}(35 \mu \mathrm{m})$ which is about $7.7 \times 10^{-8} \mathrm{~m}^{2}$ and $d_{e}$ is separation distance between the electrodes and the dispersed phase fluid which is $35 \mu \mathrm{m}$. This gives an estimated $C_{e / 2}$ to be about $0.0487 \mathrm{pF}$. Taking into account for both sides of the electrodes as they are both applied with the AC voltage, $C_{e}$ is about $0.09735 \mathrm{pF}$.

The dispersed phase fluid is separated from the ITO glass as shown in the sectional cut $B$. This can also be modelled also using the parallel plate approximation as shown in equation (3.2).

$$
C_{I}=\varepsilon_{o} \varepsilon_{r}\left(\frac{A}{d}\right)=\varepsilon_{o} \varepsilon_{r}\left(\frac{W \times L}{d_{I}}\right)
$$

However in this case, $\varepsilon_{r}$ the relative permittivity of the glass is about 7.75. The cross sectional area $A$ here refers to the width of the dispersed phase fluid $W$ multiply by 
the length $L$ taking into account only the upstream width and length which is assumed to provides a more dominant effect due to having a larger area than the downstream section. The separation distance $d_{l}$ here refers to the thickness of the glass which is about $1 \mathrm{~mm}$. Wis about $1000 \mu \mathrm{m}$ and $L$ is about $11000 \mu \mathrm{m}$. Here the calculated capacitance is about $0.754 \mathrm{pF}$.

For the dispersed phase fluid in sectional cut $\mathrm{C}$, the resistance can be estimated by using equation (3.3) where we focus on the area of the droplet formation region where the channel width and length are smaller than the upstream section (Figure 3.16).

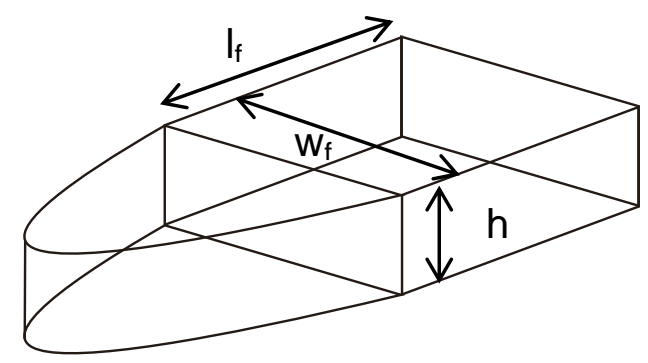

Figure 3.16: Schematic sketch of the dispersed fluid used to model the resistance of the fluid.

$$
\begin{aligned}
R_{F} & =\frac{l_{f}}{\kappa w_{f} h} \\
& =\frac{l}{\kappa}
\end{aligned}
$$

Where $I_{f}$ is the length of the fluid, $K$ is the conductivity of the fluid, $w_{f}$ is width of the channel at the region of droplet formation and $h$ is the height of the channel. Since $I_{f}$, 
$w_{f}$ and $h$ are all geometrical factors, I can be used to represent them. Here, $I$ is about $6.28 \times 10^{5} \mathrm{~m}^{-1}$.

In order to incorporate the frequency term, we take into account the impedance of each of the above components and using the voltage divider rule, the electrical circuit in figure 3.15 can be simplified as in equation (3.4).

$$
\frac{U_{t i p}}{U_{a p p}}=\left(1+\frac{1}{C_{e} / C_{\mathrm{I}}+2 \pi j(f / \kappa)\left(C_{e} / l\right)}\right)^{-1}
$$

Where $U_{t i p}$ is the voltage at the tip of the dispersed phase fluid, $U_{a p p}$ is the applied AC voltage, $C_{e}$ is the capacitance of the electrodes, $C_{l}$ is the capacitance of the ITO, $f$ is the applied frequency of the signal, $K$ is the conductivity of the dispersed phase fluid and $l$ is the geometrical equivalent in (3.3) and $j^{2}=-1$.

In the above equation (3.4), it is apparent that the rhs term is a function depending on the sole parameter of the $\mathrm{f} / \mathrm{K}$ since the other terms are geometrical and material properties. This parameter changes the $\bigcup_{\text {tip }}$ depending on the applied frequencies and conductivities of the dispersed phase fluid. This agrees well with the experimental findings obtained previously where both the applied signal frequencies and conductivities of the dispersed phase fluid affect the change in the size of the droplets when the applied AC voltage increases. This expression also changes slightly depending on the electrode configurations. For the other electrode configurations, the same voltage divider rule can be applied to the proposed electrical circuits. The potential difference between the $U_{\text {tip }}$ and the downstream pair of electrodes has to be also considered during the calculation. For example in the case of electrode in the configuration A, the potential difference is always equal to $\mathrm{U}_{\text {tip }}$ as the bottom pair of the electrodes is grounded. 


\subsubsection{Model evaluations}

In the previous section using electrodes in configuration $A$, experimental investigations on the influences of conductivities of the dispersed phase fluids are performed by fixing all the experimental parameters and varying the conductivities by five orders of magnitude (Figure 3.10). Experimental results show that when the conductivity is increased to about $2880 \mu \mathrm{S} / \mathrm{m}$ and higher, the size of the droplets formed does not change much with the increase in the applied AC voltage. No changes to the droplet formation regime are also observed. Here we use the proposed electrical model and correlation ship of the $U_{\text {tip }} / \bigcup_{\text {app }}$ to explain the findings. Calculations based on the equation (3.4) are done to verify the accuracy of the models. Figure 3.17 shows calculated $U_{\text {tip }}$ for each concentration of conductivity.

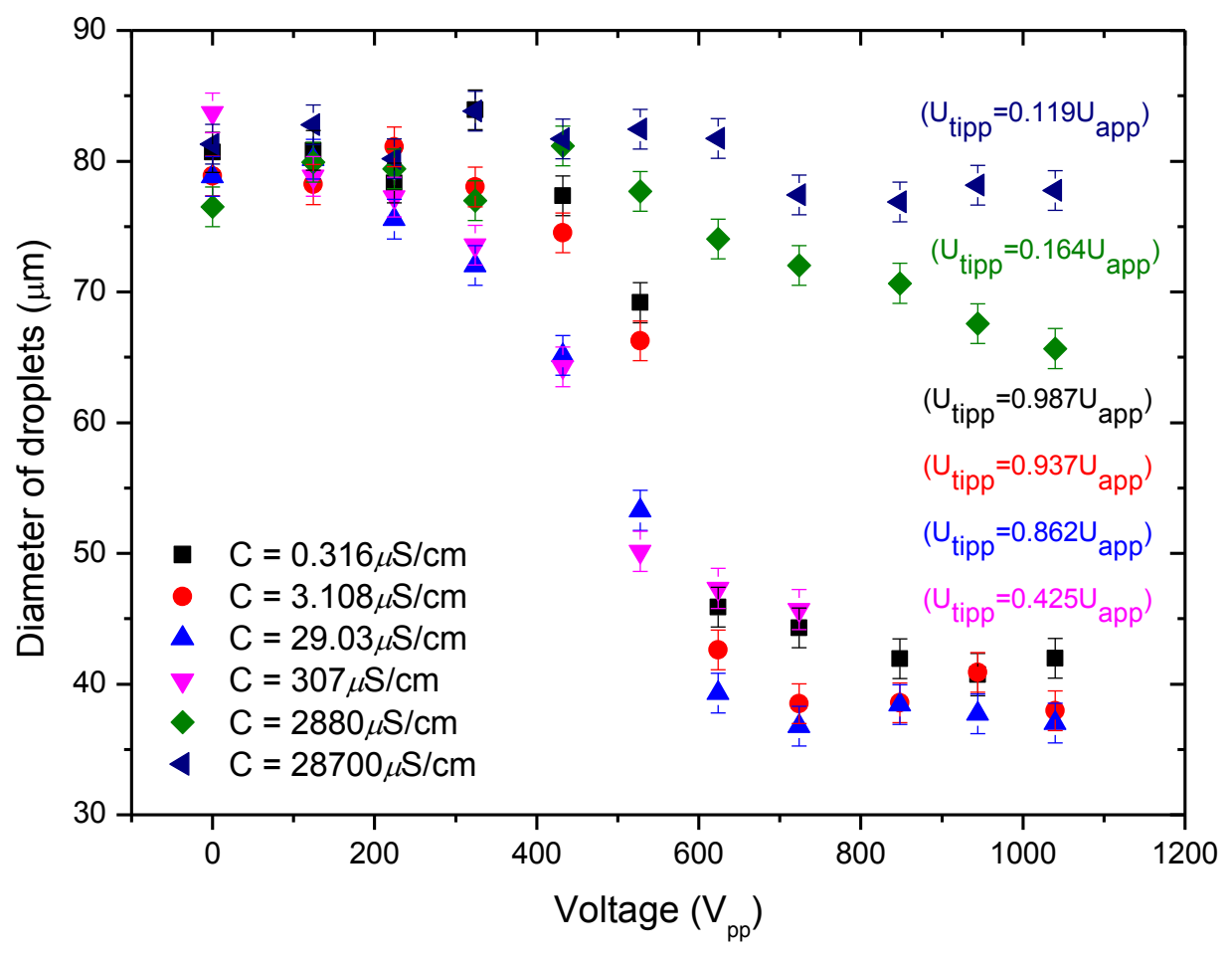

Figure 3.17: Calculations of $U_{\text {tipp }}$ for each level of conductivity. Each color represents the calculated value for each symbol. The applied signal frequency is fixed at $50 \mathrm{kHz}$. 
Using the proposed model and equation, the calculated value of $\mathrm{U}_{\text {tip }}$ can be used to explain the observations. Below $29.03 \mu \mathrm{S} / \mathrm{m}, U_{\text {tip }}$ is near to 1. Hence, slight differences are observed. For conductivities of $2880 \mu \mathrm{S} / \mathrm{m}$ and higher, the $U_{\text {tip }}$ decreases drastically to only about $16 \%$ of the $U_{\text {app. }}$. Hence, the size of the droplet does not change much when the voltage increases. However, the proposed model and equation does not explain the unstable droplet behavior and difference observed for the case when the conductivity is about $307 \mu \mathrm{S} / \mathrm{m}$.

In the previous figure 3.12 , the influence of applied frequencies at different $A C$ voltages for solutions with different conductivities was investigated. Experimental observations shows that when the milli-Q water with conductivity of about $0.3 \mu \mathrm{S} / \mathrm{cm}$ was used as the dispersed fluid, no significant differences are observed when the applied frequencies of the signal is varied from 50 to $5 \mathrm{kHz}$. When aqueous $\mathrm{NaCl}$ solution was with conductivity of about $307 \mu \mathrm{S} / \mathrm{cm}$ was used as the dispersed fluid, the change in the size of the droplets with the increase in applied AC voltage decreases with the decrease in applied frequencies of the signal. Here we used the model and equation to calculate $U_{\text {tip }}$ as shown in figure 3.18 and 3.19. For the case of milli-Q water, the calculations conform to the experimental observations. The $\mathrm{U}_{\text {tip }}$ ₹ $U_{\text {app }}$ when the frequencies are varied from 50 to $5 \mathrm{kHz}$. For the case of aqueous $\mathrm{NaCl}$ solution, the $U_{\text {tip }}$ decreases when the applied frequency decreases. At $5 \mathrm{kHz}$, the $U_{\text {tip }}$ is only about $16 \%$ of $U_{\text {app. }}$. These calculations agree well with the experimental results (figure 3.12).

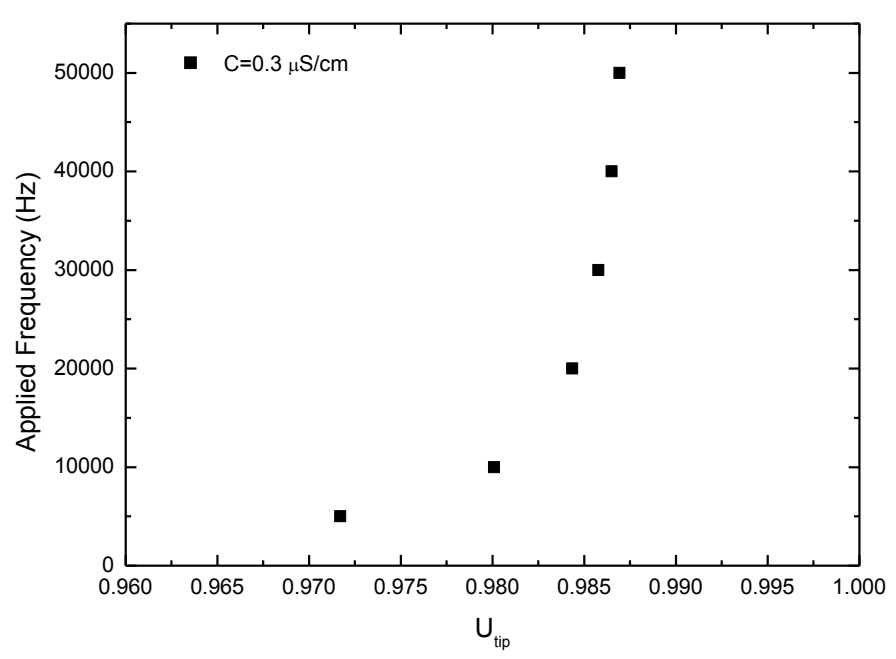


Figure 3.18: Plot of calculated $U_{\text {tip }}$ when the applied frequencies of the signal are varied from 50 to $5 \mathrm{kHz}$. This represents the case of using milli-Q water as the dispersed phase fluid.

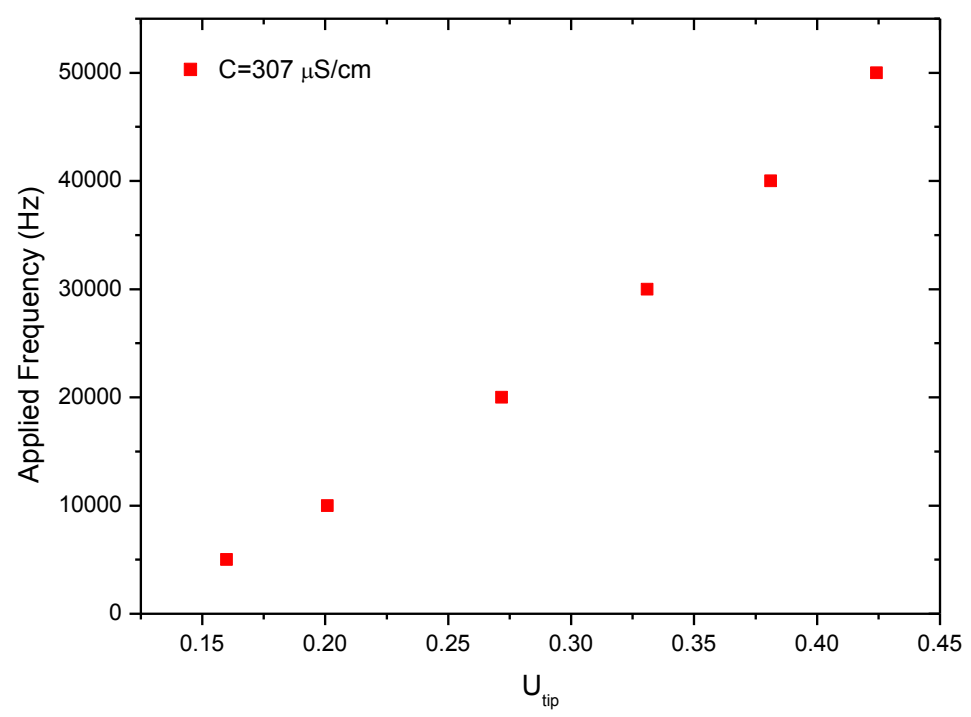

Figure 3.19: Plot of calculated $U_{\text {tip }}$ when the applied frequencies of the signal are varied from 50 to $5 \mathrm{kHz}$. This represents the case of using aqueous $\mathrm{NaCl}$ solution as the dispersed phase fluid.

\subsubsection{Rescaling of data}

The proposed electrical model and correlation ship suggests that the $U_{\text {tip }}$ depends on the sole parameter of the $f / \kappa$. Here we rescaled all the previously obtained experimental data of various conductivities and applied frequencies by using a rescaling factor $G$. This is done with first using the set of data for using the milli-Q water with conductivity of about $0.3 \mu \mathrm{S} / \mathrm{cm}$ and varying the frequencies between 50 to $5 \mathrm{kHz}$ as a reference. The other sets of data are then shifted to enable the collapse to form a master curve. Then we plot the rescaling factor $G$ with $f / K$ in the inset for selected cases to determine the relationship of the system. The rescaling plot is depicted in figure 3.20 . 


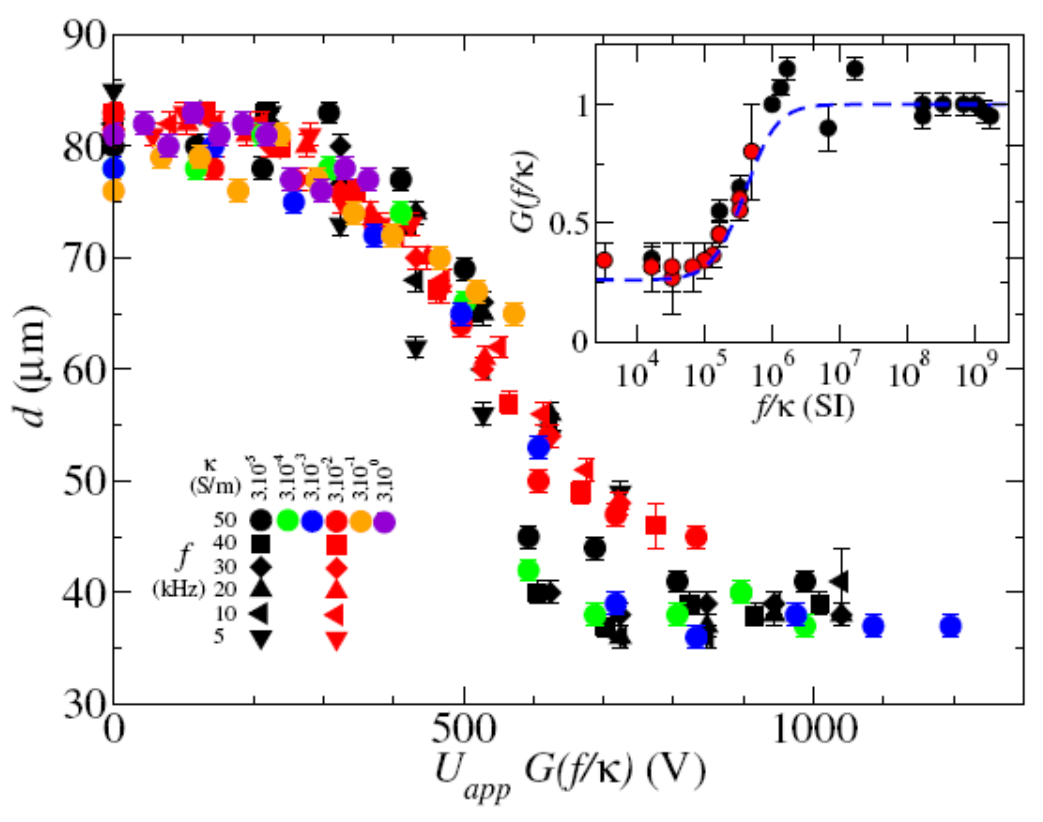

Figure 3.20: Droplet diameter as a function of the rescaled voltage. Inset: The rescaling factor $G$ is plotted as a function of $f / \kappa$ for selected cases. The blue dash line is a fit to equation (3.4). The black points are obtained from regime 1 and the red points are obtained from regime 3 .

The inset plot obtained above suggested that the system behaviors as a high pass filter with a cut-off at around $f / \kappa=4 \times 10^{5} \mathrm{~m} / \mathrm{F}$. At $\mathrm{f} / \mathrm{K}$ of about above $10^{6}$, the rescaling factor is about 1 and hence little changes are observed to $U_{\text {tip. }}$. However below the cut off of $f / \kappa=4 \times 10^{5} \mathrm{~m} / F$, the $U_{\text {tip }}$ is only about $25 \%$ of the $U_{\text {app }}$. This rescaling agrees well with observed findings in the previous section.

\subsubsection{Phase diagrams}

In order to further assess our proposed electrical model and equation (3.4), we investigate the droplet production mechanism at a fixed applied voltage of $1 \mathrm{kV}$ for electrode configurations $A, B$ and $C$. A fixed volumetric flow rate of $Q_{c}: Q_{d}: Q_{c}=$ 200:50:200 $\mu \mathrm{l} / \mathrm{hr}$ is used for all the experiments. The frequencies of the applied signals are varied from 50 to $1 \mathrm{kHz}$. For electrode configurations $\mathrm{B}$ and $\mathrm{C}$, we modified slightly the electrical circuits to fit the actual connection system. Figure 3.21 to 3.23 illustrates the obtained phase diagrams. 

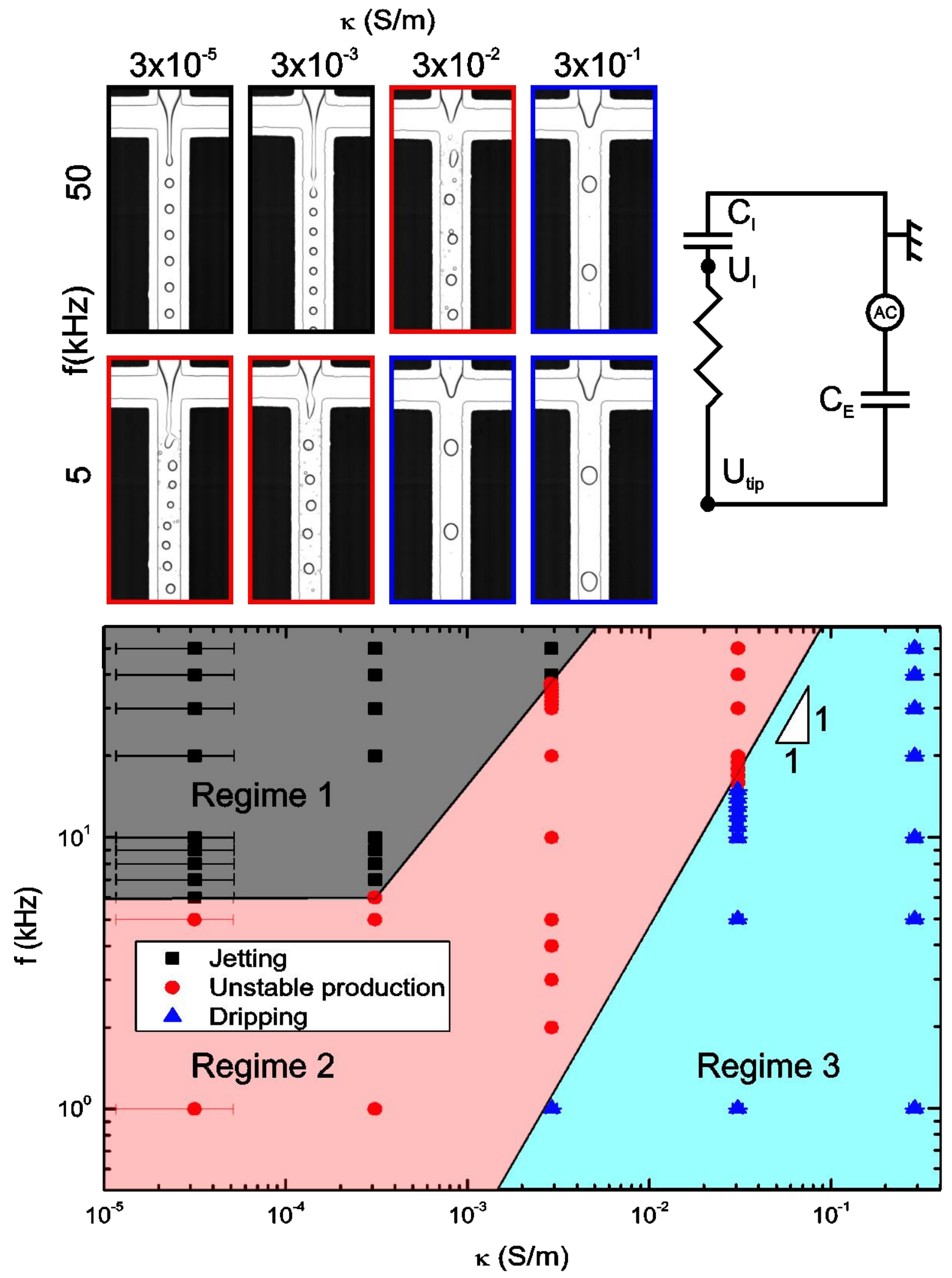

Figure 3.21: Phase diagram for electrode configuration $A$ at different applied frequencies. The applied AC voltage is fixed at $1 \mathrm{kV}$. 
$\kappa(\mathrm{S} / \mathrm{m})$
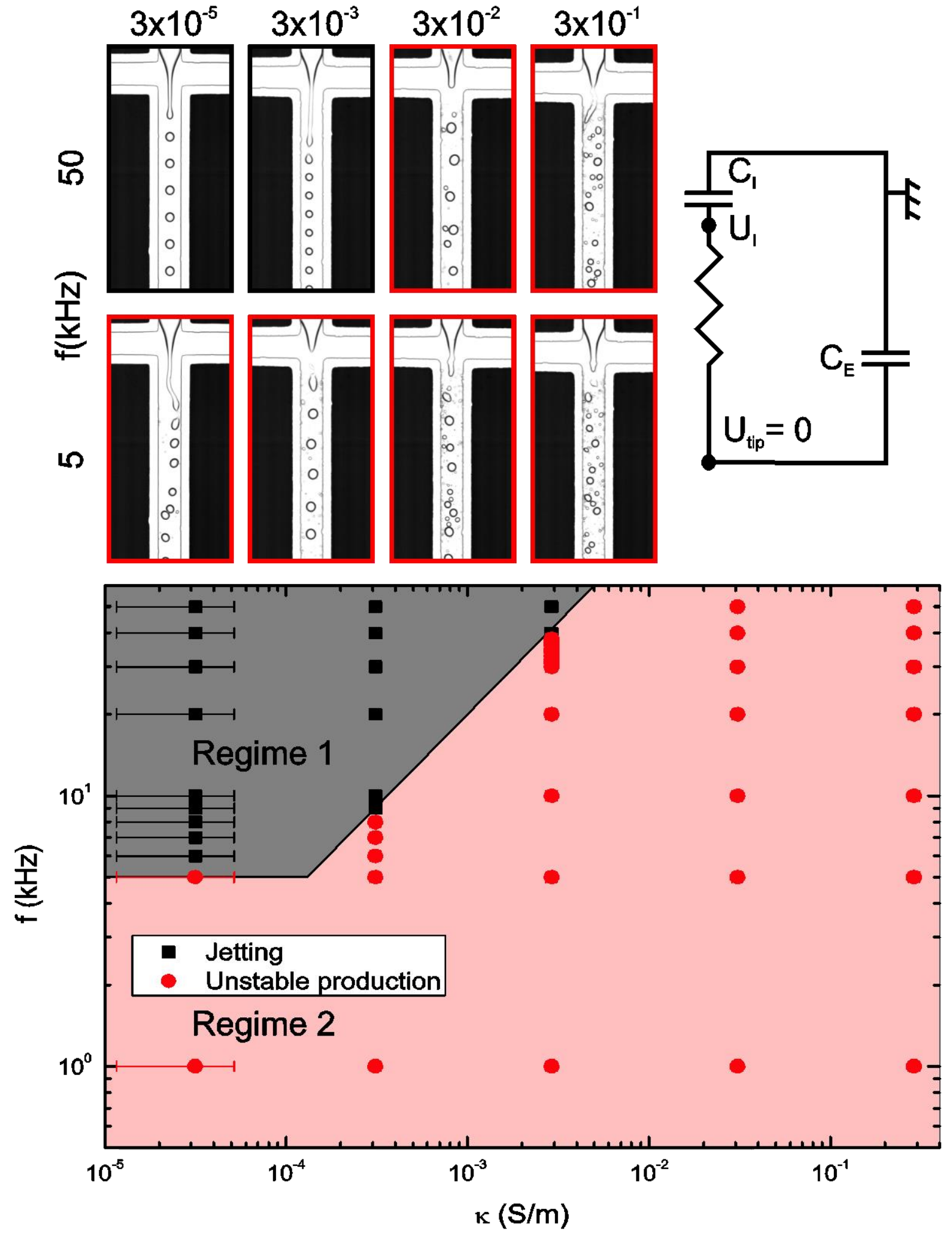

Figure 3.22: Phase diagram for electrode configuration $B$ at different applied frequencies. The applied AC voltage is fixed at $1 \mathrm{kV}$. The modified electrical circuit is shown above. 

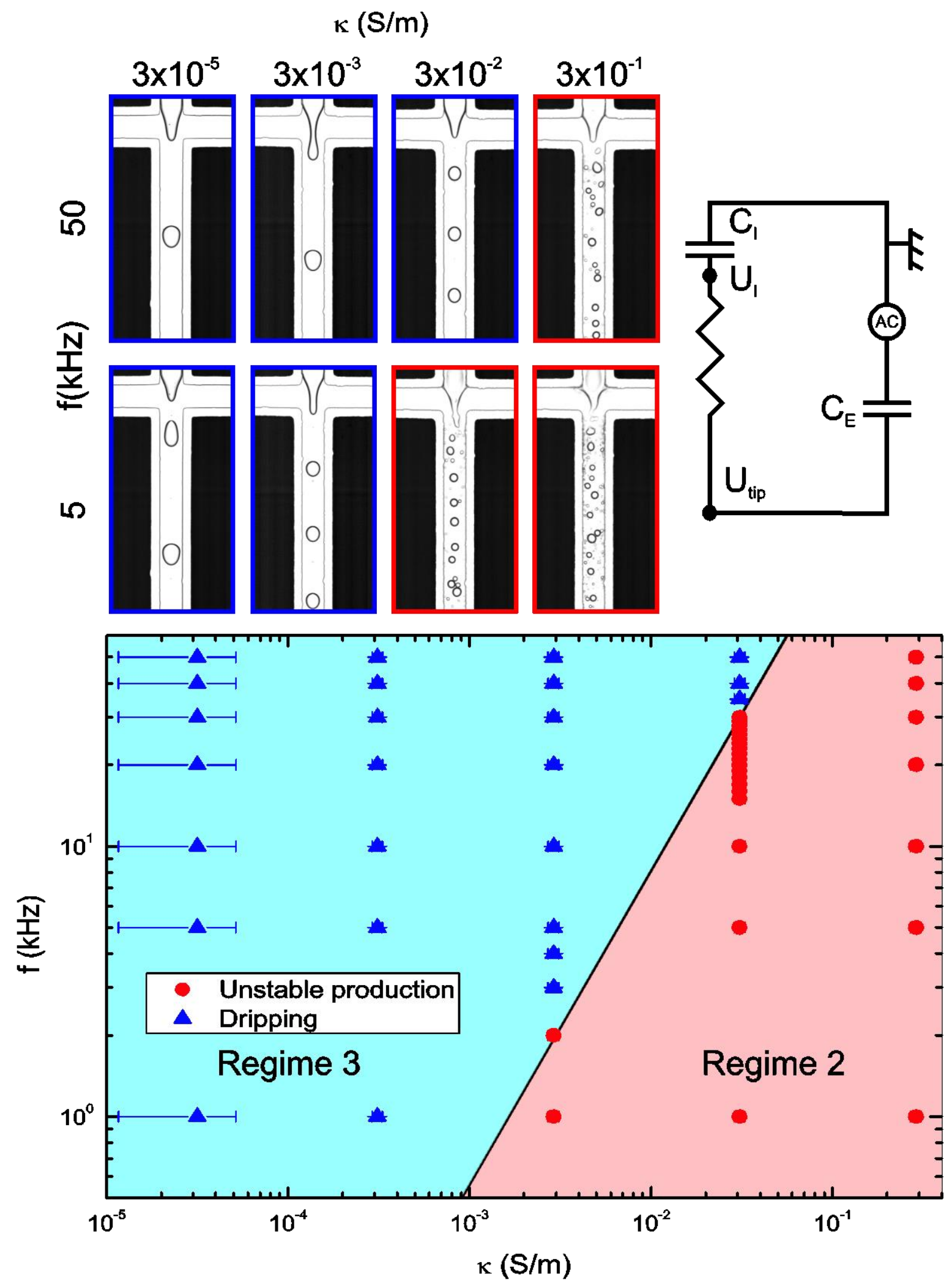

Figure 3.23: Phase diagram for electrode configuration $C$ at different applied frequencies. The applied AC voltage is fixed at $1 \mathrm{kV}$. The modified electrical circuit is shown above. 
In the above phase diagrams, 3 distinct droplet formations are observed which can be classified as regime 1,2 and 3. In regime 1, it is classified as the stable jetting mode where monodispersed droplets are by formed by the classical Rayleigh plateau instability. This regime occurs generally at low conductivities and high applied frequencies as seen in figure 3.21 and 3.22 for electrode configuration $A$ and $B$. In regime 2, it is classified as the unstable droplet production mode which is similar to whipping instabilities observed in the electrospraying experiments [60, 97]. This regime occurs in all electrodes configuration $A, B$ and $C$. However, the occurrence differs at different level of conductivities and applied frequencies for each electrode configuration. In regime 3 , droplets are observed to form via a dripping mode. In this mode, the calculated $U_{\text {tip }}$ is much lower than the $U_{\text {app }}$ (about $25 \%$ according to calculations) which in turns suppressed the magnitude of the electric field. This regime only occurs in electrode configuration $A$ and $C$ at different level of conductivities and applied frequencies for each electrode configuration.

In the previous figure 3.20, we observed a cut-off at around $\mathrm{f} / \mathrm{k}=4 \times 10^{5} \mathrm{~m} / \mathrm{F}$ in the inset where the system behave like a high pass filter. Interestingly, the lines separating regime 2 and 3 in electrode configuration $A$ also corresponds to the value of $\mathrm{f} / \mathrm{K}=5 \times 10^{5} \mathrm{~m} / \mathrm{F}$ which is close to the cut off condition. The value is obtained by calculating the slope of the lines separating the regimes. This observation ascertained that the system behaviors as a high pass filter when in the electrode configuration A. However, we are still unable to explain the rationale between the transitions from regime 1 to 2 .

In order to further assess our proposed electrical models and circuits, we focus now on the electrode configuration B. Firstly, regime 1 in this case has disappeared and droplets are formed mainly in regime 2 and 3 at different levels of conductivities and applied frequencies. According to the electrical circuit, the $U_{\text {tip }}$ in this case is always equal to 0 while the $U_{\text {app }}$ at the downstream pair of electrodes is always equal $U_{\text {app }}$. In this case, the condition for the system to be in regime 3 is never fulfilled and the system no longer behaves as a high pass filter. This explains the disappearance of regime 3 in this particular electrode configuration.

In electrode configuration $\mathrm{C}$, the electrical circuit is similar to the one as in electrode configuration $A$ where the $U_{\text {tip }}$ can be approximated by using the same equation (3.4). 
However in this case, as the pair of electrodes in the downstream is also applied with the same $A C$ voltage, there is no significant voltage difference between the $U_{\text {tip }}$ and the downstream pair of electrodes. Hence, this explains the disappearance of regime 1 where there is a significant voltage difference between $U_{\text {tip }}$ and the downstream pair of electrodes which is grounded. In this configuration, we also observed a transition between regime 2 and 3 at a value of $f / K=5 \times 10^{5} \mathrm{~m} / \mathrm{F}$ which agrees to the previous calculations.

\subsection{Electrohydrodynamic model}

In this section, we propose an electrohydrodynamic model to explain the coupling effect of both the electric field and the hydrodynamic flow. We will focus on using the proposed model to evaluate the experimental results obtained in figure 3.7. The electrodes are in configuration $A$ and volumetric flow rates flow rate ratio is fixed at 8 .

In the absence of the electric field, the diameter of the droplets formed can be correlated with the reciprocal of the capillary number $[17,73,78]$. The capillary number can be as described in equation (3.5).

$$
C a=\frac{\eta V}{\gamma}=\frac{\eta Q_{c}}{w h \gamma}
$$

Where $\eta$ is the dynamic viscosity of the continuous phase fluid, $V$ is the characteristic velocity of the fluid and $\gamma$ is the interfacial tension of the fluid. The characteristic velocity of the fluid $V$ can be further expressed as the $\left(Q_{c} / w h\right)$ where $w$ is the width of the continuous phase channel and $\mathrm{h}$ is the height of the channel.

In the presence of the electric field, a mechanical effect acts at the interface between the fluids as a result of the Maxwell stress. The Maxwell stress is the electrical 
pressure normal to the interface which acts in the opposition direction to the Laplace pressure. This effect can also be expressed in terms of the electric bond by comparing the relative weight of the Laplace pressure and the Maxwell stress as described in equation (3.6).

$$
B_{e}=\frac{\varepsilon_{0} \varepsilon_{r} U^{2} w k}{\gamma\left(2 d_{e}+w\right)^{2}}
$$

Where $\varepsilon_{o}$ is the vacuum permittivity which is about $8.854 \times 10^{-12} \mathrm{~F} / \mathrm{m}, \varepsilon_{r}$ is the relative permittivity of the oil which is about 2.1. $U$ is the rms voltage, $w$ is the width of the channel and $k$ is a fitting parameter to account for the curvature of the droplet. $\gamma$ is the interfacial tension between the fluids and $d_{e}$ is the distance of the electrodes to the fluidic channel which is $35 \mu \mathrm{m}$ in this case. The electric bond number in the calculations range between 0.2 to about 0.31 .

In order to couple both the dimensionless numbers to account for both the hydrodynamic and Maxwell stresses, we introduce here the effective capillary number which can be described in equation (3.7).

$$
C a_{e f f}=\frac{C a}{1-B_{e}}
$$

Figure 3.24 illustrates the logarithmic plot of the diameters of the droplets against the proposed effective capillary number. When the applied voltage is zero, the electric bond number will be zero and the correlation reverts back to the pure hydrodynamic case. 


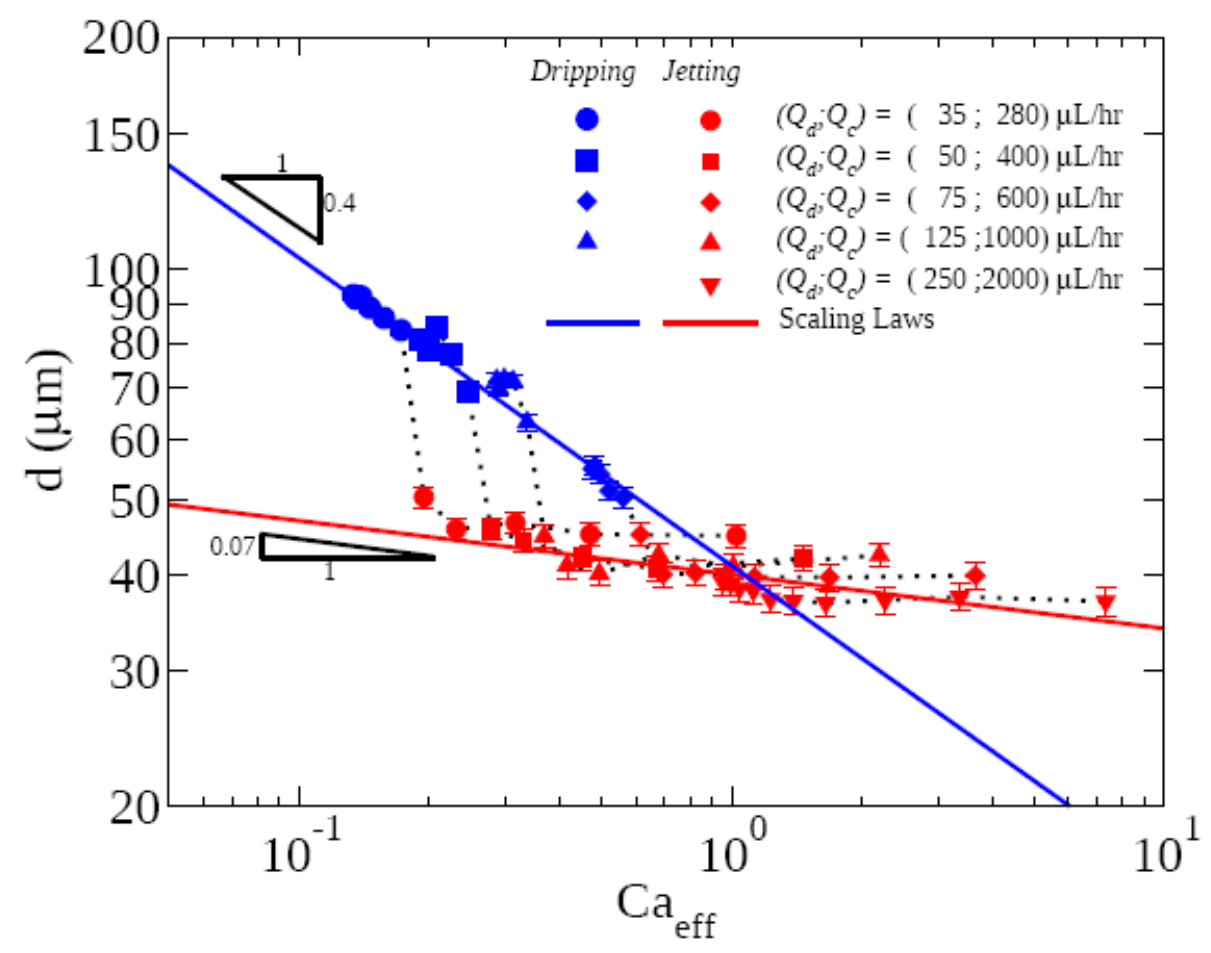

Figure 3.24: Diameters of the droplets as a function of the effective capillary number. The continuous phase volumetric flow rate has been expressed here as the total volumetric flow rate of the continuous phase fluid for clarity and comparison.

From the plot, we obtained a power law where the $\mathrm{d} \propto \mathrm{Ca}^{-0.4}$ for the pure hydrodynamic case. This deviation from $\mathrm{d} \propto \mathrm{Ca}^{-1}$ is expected as the power law depends on various factors such as volumetric flow rate ratio, aspect ratio of the channels and viscosity ratio. In our case, the aspect ratio of the channel is about 2.85 and the viscosity ratio between the two fluids is about 30 . Moreover, this model account only on the shearing of the fluids during the formation of the droplets [73].

Interestingly, when $\mathrm{k}=0.5$ is used as the fitting parameter, a similar power law of $\mathrm{d} \alpha$ $\mathrm{Ca}_{\text {eff }}{ }^{-0.4}$ is obtained for droplets formed in the dripping regime. For the case when droplets are formed in the jetting regime, a power law of $d \propto \mathrm{Ca}_{\text {eff }}{ }^{-0.07}$ is obtained. This agrees with the literature where a higher dependence on the capillary number is expected when droplets are formed in the dripping regime and a lower dependence when in the jetting regime [87]. In the jetting regime, the droplets break up occurs at 
the downstream of the channel whereas in the dripping regime the droplets break up in the upstream channel.

In figure 3.24, we also observed that the transition between the dripping to the jetting regime is not captured by the $\mathrm{Ca}_{\text {eff }}$ number. In the pure hydrodynamic case, the transition between the dripping to the jetting regime occurs at about $\mathrm{Ca} \approx 1$. However, for the electrohydrodynamic case, the transitions occur at different $\mathrm{Ca}_{\text {eff }}$ numbers for all the tested volumetric flow rates. These transition numbers is also much lower than the pure hydrodynamic case.

\subsection{Discussion}

The proposed electrical and electrohydrodynamic models do not fully explain the system and have several limitations. In the electrical modelling of the system, the proposed electrical representations for the electrodes in different configurations do not explain the transition from the stable jetting to the unstable droplet production regime (regime 1 to regime 2). Moreover, several different and distinct instabilities can be observed within the proposed single unstable droplet production regime. This can be further classified in order to elucidate or isolate the different causes of instabilities.

The effective capillary number does not address or account for the complex nature of the interfacial tensions namely the absorption kinetics at the interface of the fluids. The model simply assumed that the interfacial tension is at equilibrium while neglecting that the dynamic interfacial tension should be the one accounting for the size of the droplets during the droplet formation process. While the use of surfactants is practical in most applications, this introduces an additional level of complexity which should be addressed. Additional experiments without the use of surfactants and with different concentration of surfactants will present a more comprehensive understanding on the scaling mechanism.

Last but not least, the models do not address other important dimensionless numbers such as the viscosity ratio between the fluids, aspect ratio of the channels 
and volumetric flow rate of the dispersed phase. Systematic studies on variations to these parameters will allow the proposed models to be better characterized and understood.

\subsection{Conclusion}

In conclusion, we developed and characterized an AC voltage induced electric field to actively control the size of the droplets formed in a microfluidic flow focusing device. Experimental results investigating various parameters are presented and discussed with the proposed models. The electrical models address the issue of the voltage at the tip of the dispersed phase fluid. The voltage at the tip changes depending on the electrode configurations and the ratio of $f / k$. In the case of electrode in configuration $A$, the system acts as a high-pass filter at a cut off of about $\mathrm{f} / \mathrm{k}=4 \times 10^{5} \mathrm{~m} / \mathrm{F}$. The electrohydrodynamic model uses the effective capillary number to account for both electrical and hydrodynamic effect by taking account the effect of hydrodynamic and Maxwell stresses. Different power laws are obtained to relate the diameters of the droplet formed in different droplet formation regime. However, the model does not take into account the absorption kinetics of the surfactants and also the transition between the dripping and the jetting regime. 


\section{Part 3: Dynamic studies}

\section{Chapter 4: The Microfluidic Jukebox}

Say Hwa Tan, Florine Maes, Benoit Semin, Jeremy Vrignon and Jean-Christophe Baret.

\section{Content}

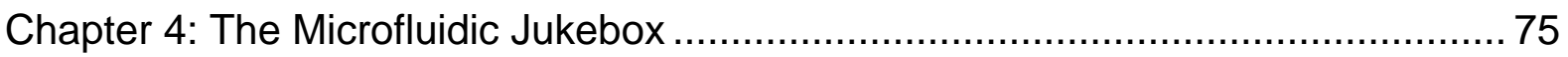

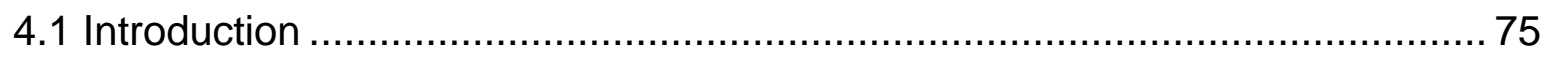

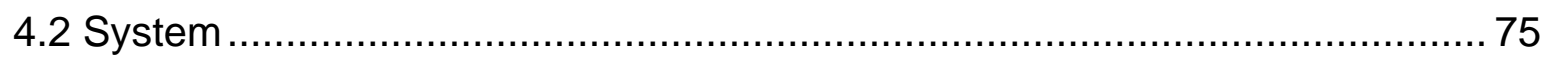

4.2.1 Electrical Control of droplet production .............................................. 75

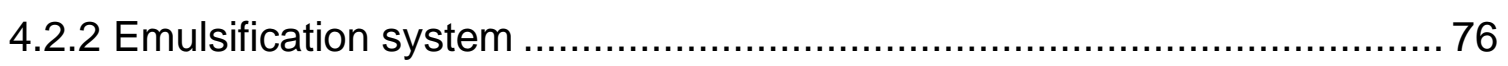

4.2.3 Optical setup for detection ............................................................... 76

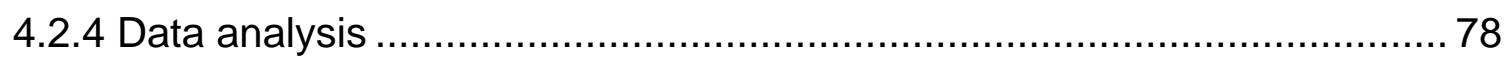

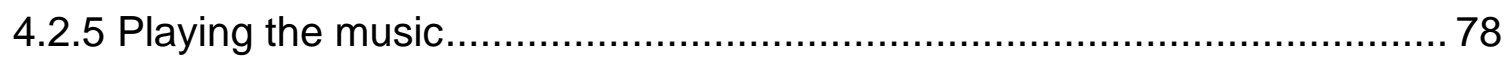

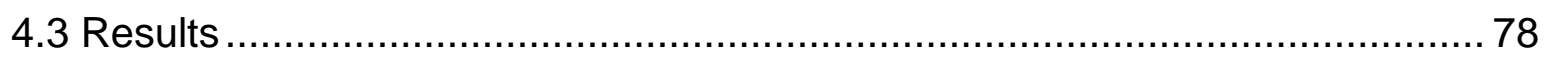

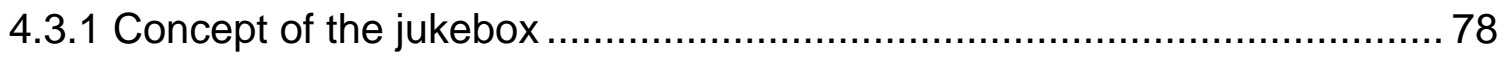

4.3.2 Frequency range and musical notes ...................................................... 79

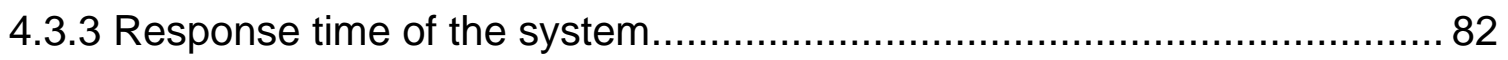

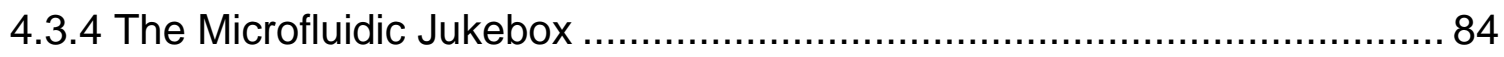

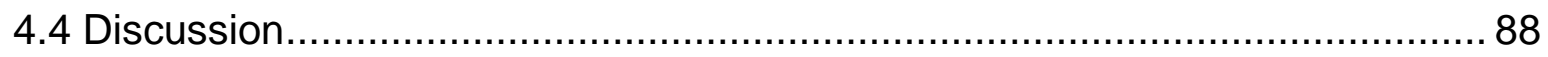

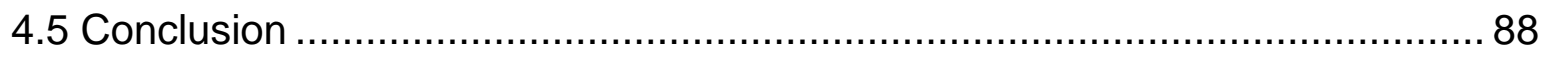


This chapter is described in the following publication:

The Microfluidic Jukebox [98].

Scientific Reports 4, Article number: 4787, doi: 10.1038/srep04787

Awarded the Merit Prize in the Tan Kah Kee Young Inventor's Award 2014

Droplets, Membranes \& Interfaces

Tröpfchen, Membranen \& Grenzflächen

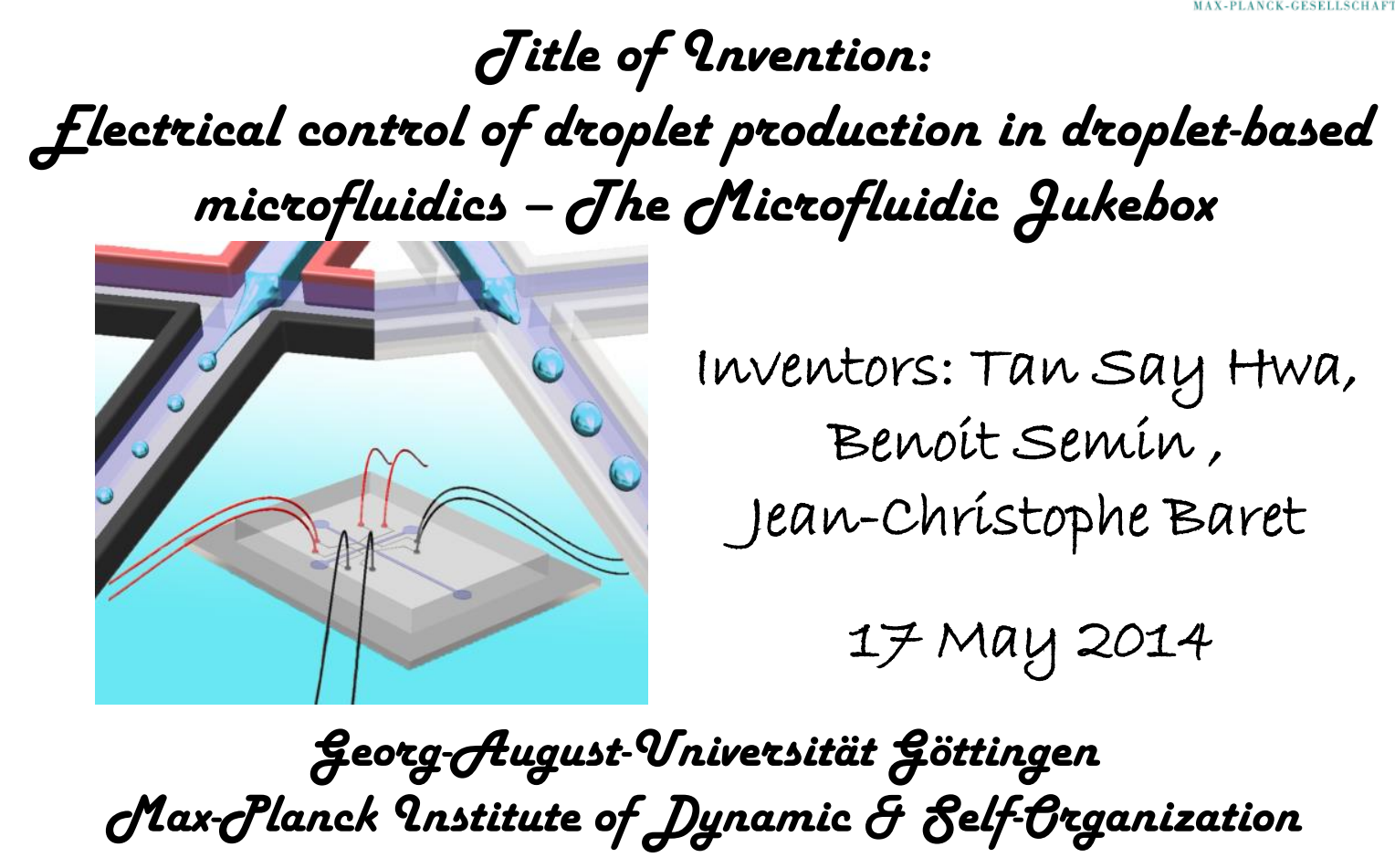

Press releases on this article:

http://www.mpg.de/8266925/microfluidic chip music

http://www.cafe-sciences.org/billets/tag/microfluidique/

http://www.nanowerk.com/nanotechnology-news/newsid=35977.php

http://www.innovations-report.de/html/berichte/biowissenschaften-chemie/musikunter-dem-mikroskop.html 


\section{Chapter 4: The Microfluidic Jukebox}

\subsection{Introduction}

In the previous studies, we studied the static properties of the system and investigate the influences of various different parameters. We proposed different electric models to represent the 3 main electrode configurations. The voltage at the tip can be calculated by using the voltage divider rule and taking into account the ratio of the $\mathrm{f} / \mathrm{K}$. An electrohydrodynamic model was also proposed to account for both the hydrodynamic and Maxwell stresses.

In this chapter, we use electrode configuration A to study the dynamic effect of the system through the production of musical sound tracks. The external control provided by the $A C$ voltage enables the size and hence the frequencies of the droplets generated to be modulated rapidly through mass conservation. Capitalizing on this fast control, a transducing method converts the droplet frequencies into audible sound tracks. We also examine the response time of the system and show that the fast switching capabilities is suitable for the propose application. Last but not least, we present the microfluidic jukebox to play two different musical pieces namely "Ode to Joy" and "The flight of the Bumblebee".

\subsection{System}

\subsubsection{Electrical Control of droplet production}

Microfluidic devices which were similar to the previous chapter were used in this study. The electrical system is setup to be in configuration A where the top pair of the electrodes is applied the AC voltage while the bottom pair and the ITO are grounded. A fixed sinusoidal signal of $50 \mathrm{kHz}$ was used in all the experiments. The amplification ratio between the applied $A C$ voltage and the final amplified voltage at this frequency is about $490(\mathrm{U}=490 \times \mathrm{u})$. Here, $U$ represents the final amplified 
voltage in peak to peak and $u$ is the peak to peak voltage produce by the signal generator.

\subsubsection{Emulsification system}

Water-in-oil (W/O) droplets are produced by flowing milli-Q water mixed with $10 \mu \mathrm{M}$ of fluorescein as the dispersed phase fluid $\left(Q_{d}\right)$ in the middle of the channel. The conductivity of the dispersed phase fluid is about $2.2 \mu \mathrm{S} / \mathrm{cm}$ measured with a commercial conductivity meter (Eutech 2700, Thermo Fisher Scientific). Mineral oil (M5904, Sigma Aldrich) with 5\% wt/wt of non-ionic surfactant (SPAN 80, Sigma Aldrich) flows in the two side channels as the continuous phase fluid $\left(Q_{c}\right)$. The equilibrium interfacial tension between the fluids measured is about $3.5 \mathrm{mN} / \mathrm{m}$ as shown in figure 3.6 previously.

\subsubsection{Optical setup for detection}

A standard epi-fluorescence optical setup is used for the detection of droplets [12]. In order to detect the droplets, the microfluidic device is first mounted on an inverted microscope (IX81, Olympus). A $473 \mathrm{~nm}$ blue laser with a power of about $20 \mathrm{~mW}$ is directed through the objective of the microscope and focused into the downstream channel where the generated droplets are flowing. When a droplet passes through the laser, it emits fluorescence light. The fluorescence signal is then transmitted by the dichroic which also reflects the backscattered laser light. A notch filter absorbs the remaining scattered light in order to retain only the fluorescence signal. This filtered signal is then reflected on a second dichroic towards a photomultiplier tube (PMT). The PMT (ALR 3003ELC) gain is controlled by a 0.1-0.4 V voltage source (AL841BELC). The PMT output is then connected to the computer through the audio card which digitalized the fluorescence signal (AD converter; $44.1 \mathrm{kHz}$ sampling rate and 16 bits depth). The experimental videos are captured using a high speed camera (V311, Phantom). Figure 4.1 illustrates the optical setup. 


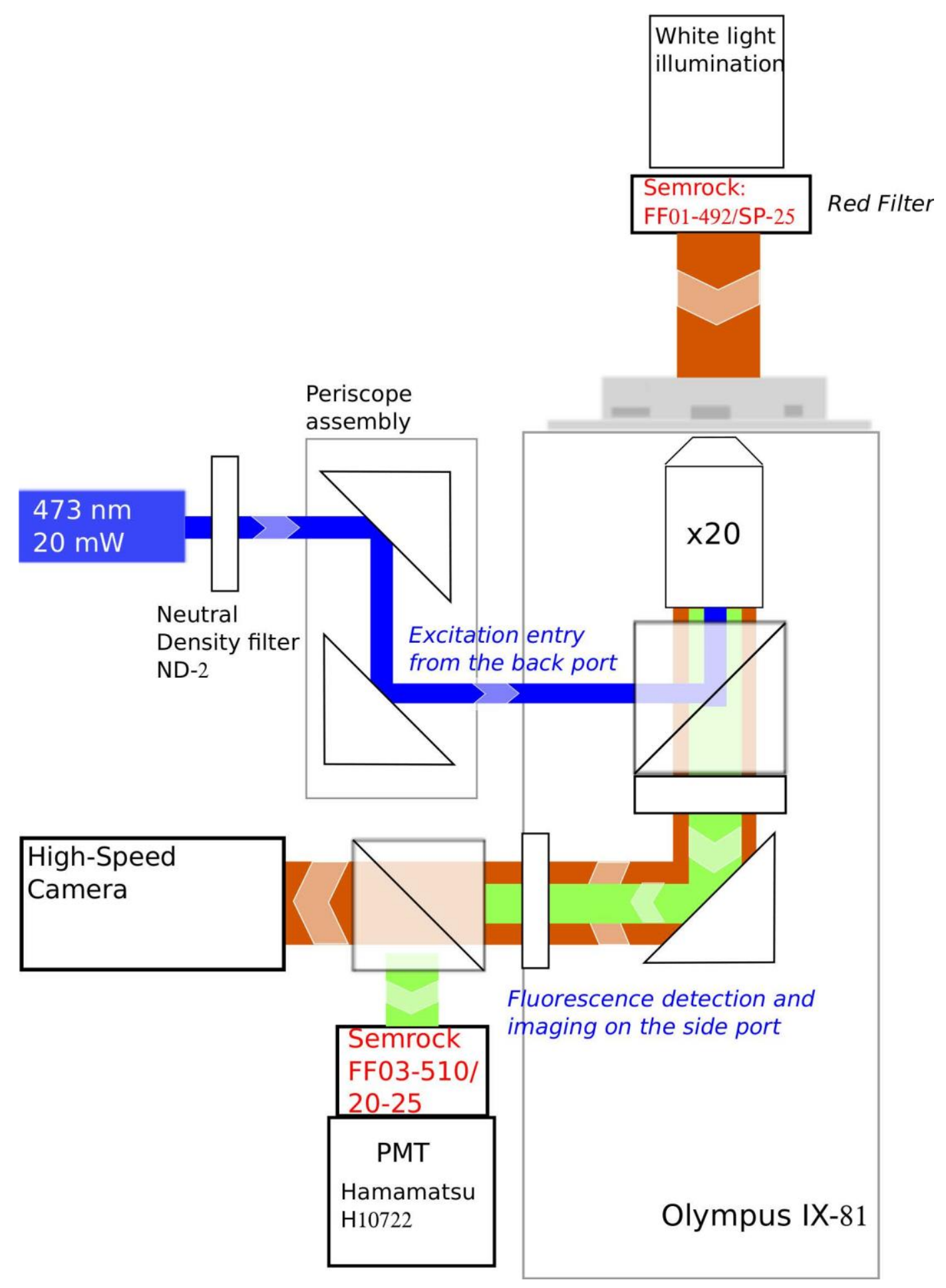

Figure 4.1: Illustration of the optical setup for the detection of droplets. 


\subsubsection{Data analysis}

In order to obtain the frequencies of the generated droplets, a Fast Fourier Transform (FFT) algorithm based on 16384 samples with a spectrogram of $2.7 \mathrm{~Hz}$ is used to analyze the signal. The droplet production frequencies are estimated by extracting the first partial (fundamentals) of the recorded PMT signal.

\subsubsection{Playing the music}

In order to play the required musical sound tracks, a calibration is first performed to obtain the relationship between the applied voltage and the resulting droplet generated frequencies. Musical scores are obtained from www.abcnotes.com. A home-made perl program then convert the musical scores into series of frequencies based on the equal temperament scale. The score is adapted by shifting it by an integer number of half tones to fit the accessible range of frequencies.

\subsection{Results}

\subsubsection{Concept of the jukebox}

We use a transducing method to convert the droplet production frequencies into audible musical notes. Water-in-oil droplets are first generated using fixed volumetric flow rates for both the dispersed and continuous phase fluids. A laser is focused into the channel and the epi-fluorescence emitted by the droplets is detected by the PMT. The electrical signal from the PMT output is then analyze using FFT to obtain the fundamental frequency of the droplet generation. The output is then directly recorded using a standard computer sound card. In order to avoid saturation, the PMT gain is tuned to have fluorescence amplitudes which are smaller than $0.4 \mathrm{~V}$. Figure 4.2 illustrates the conceptual trial for the microfluidic jukebox. 

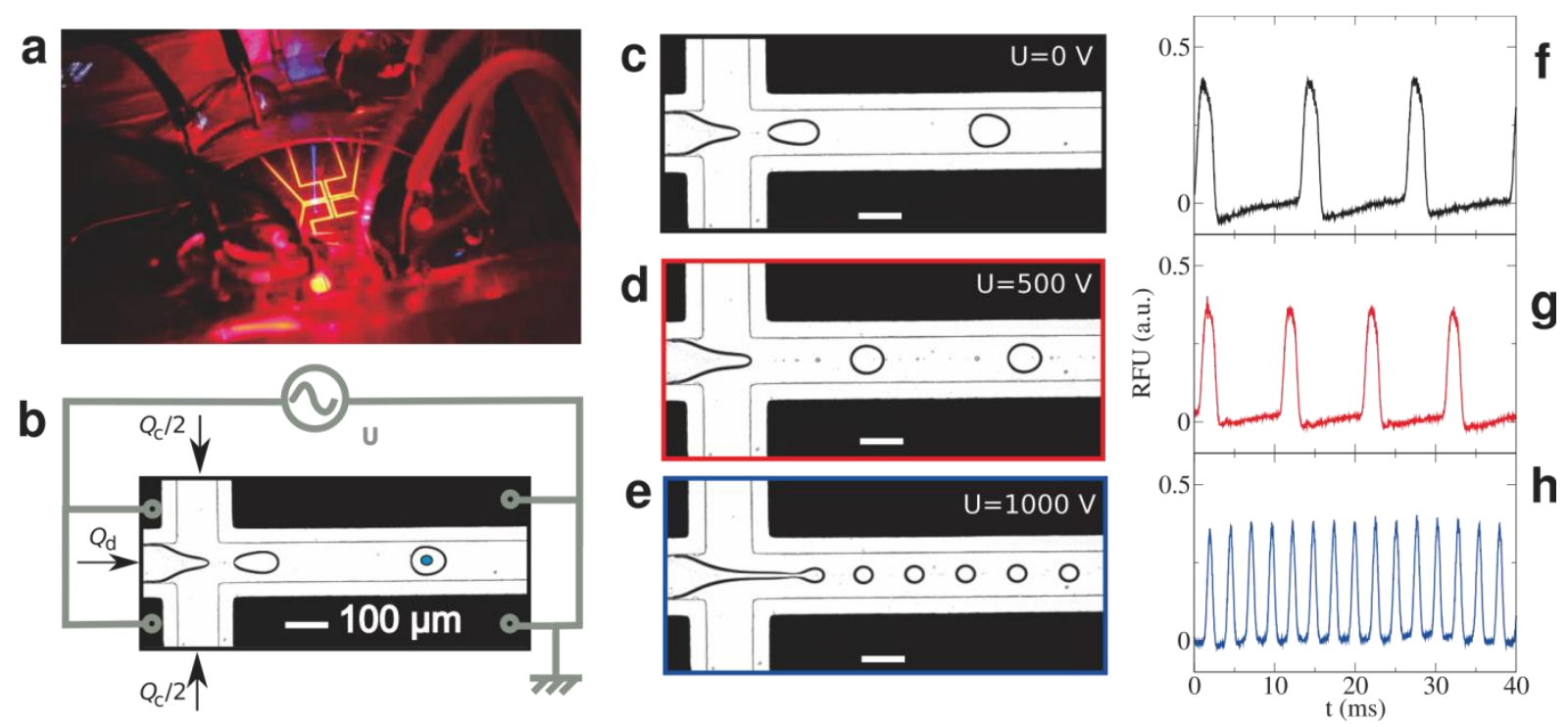

Figure 4.2: Concept of the microfluidic jukebox. (a) Microfluidic device mounted on the optical detection setup. The yellow reflections are the electrodes and the blue line is the laser focused into the channel. (b) Snapshot of the droplet formation with the electrical connections. (c-e) Snapshots of the droplets generated taken at various applied voltages. The volumetric flow rates are fixed at $Q_{d}=50 \mu \mathrm{l} / \mathrm{hr}$ and $Q_{c}=400$ $\mu \mathrm{l} / \mathrm{hr}$. (f-h) The corresponding fluorescence signal recorded by the sound card. Each peak represents one droplet flowing through the laser.

\subsubsection{Frequency range and musical notes}

We first converted the musical notes into frequencies using the equal temperament scale as shown in figure 4.3. This scale provides a fixed relationship between the frequencies and the musical notes. In this scale, the ratio between two successive half tones is $2^{1 / 12}$. The notes at higher or lower frequencies are obtained by multiplying (higher octave) or dividing (lower octave) the frequencies by a factor of 2 . A comparison between the equal temperament scale and the just intonation shows that the frequency shift between a note in both scale is at most $2 \%$. 


\begin{tabular}{cccc}
\multicolumn{3}{c}{ Note } & Note Just intonation Equal temperament \\
\hline \hline DO & C & 264,00 & 261,63 \\
DO & C & 275,00 & 277,18 \\
RE & D & 297,00 & 293,66 \\
MI b & E & 316,80 & 311,13 \\
MI & E & 330,00 & 329,63 \\
FA & F & 352,00 & 349,23 \\
FA & F & 371,25 & 369,99 \\
SOL & G & 396,00 & 392,00 \\
SOL & G & 412,50 & 415,30 \\
LA & A & 440,00 & 440,00 \\
SI b & B $b$ & 475,20 & 466,16 \\
SI & B & 495,00 & 493,88 \\
DO & C & 528,00 & 523,25
\end{tabular}

Figure 4.3: Relationship between the musical notes and frequencies in two different classical scales.

In order to validate the stability of the musical notes produce at the particular frequency, we first produce two different musical notes for several seconds using different AC voltages. The volumetric flow rates are fixed at $Q_{d}=85 \mu \mathrm{l} / \mathrm{hr}$ and $Q_{c}=$ $270 \mu / / h r$. At this flow rate, when the applied $A C$ voltage is $u=0.05 \mathrm{~V}$ a $C$ note is produce and $\mathrm{G}$ note is produce when $\mathrm{u}=0.845 \mathrm{~V}$. Varying the applied $\mathrm{AC}$ voltage from 0.01 to $2.15 \mathrm{~V}$, the droplet generation frequencies varies from $180 \mathrm{~Hz}$ to $700 \mathrm{~Hz}$. This frequency range allows all the notes in a scale to be accessible for the playing of musical pieces. Applying the voltages incrementally or at random, the same relationship between the applied voltage and detected frequency is obtained. This indicates that system has no hysteresis in the electrical actuation. The results are also reproducible from devices to devices with only a small variation in the shape of the curves. We believe that the small variation is due to the variation of the microfabrication technique. Figure 4.4 shows the measurement and calibration results obtained. 

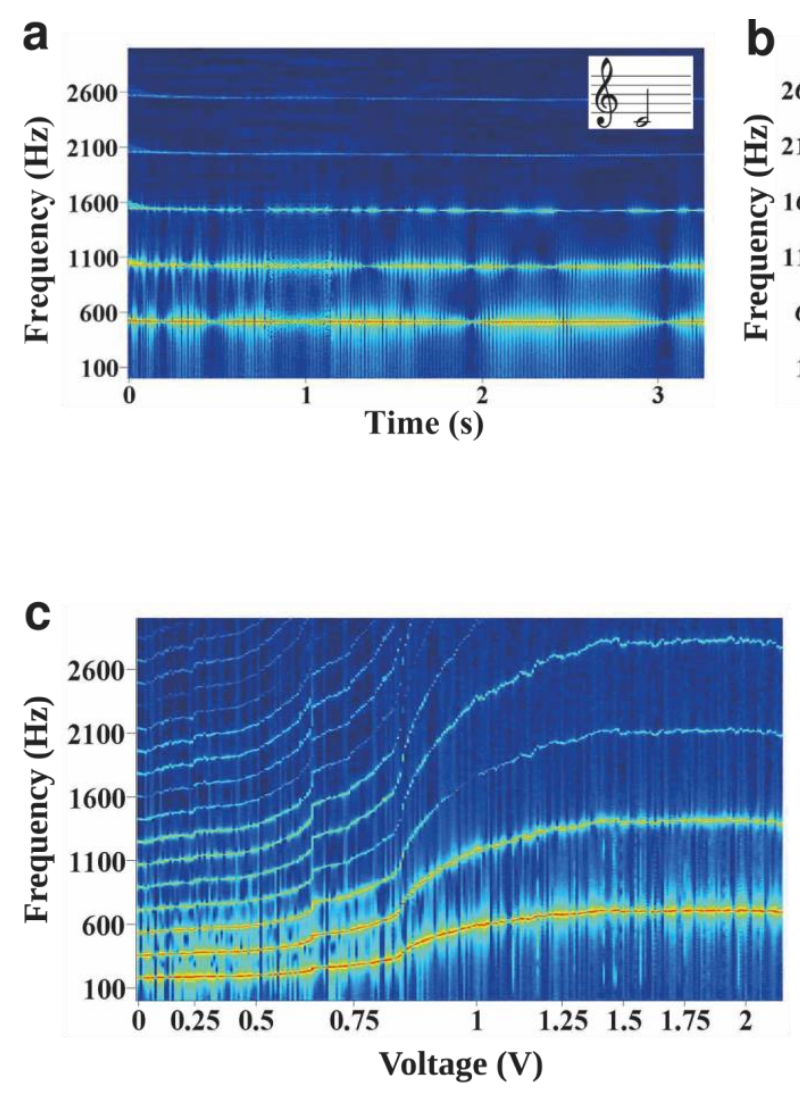
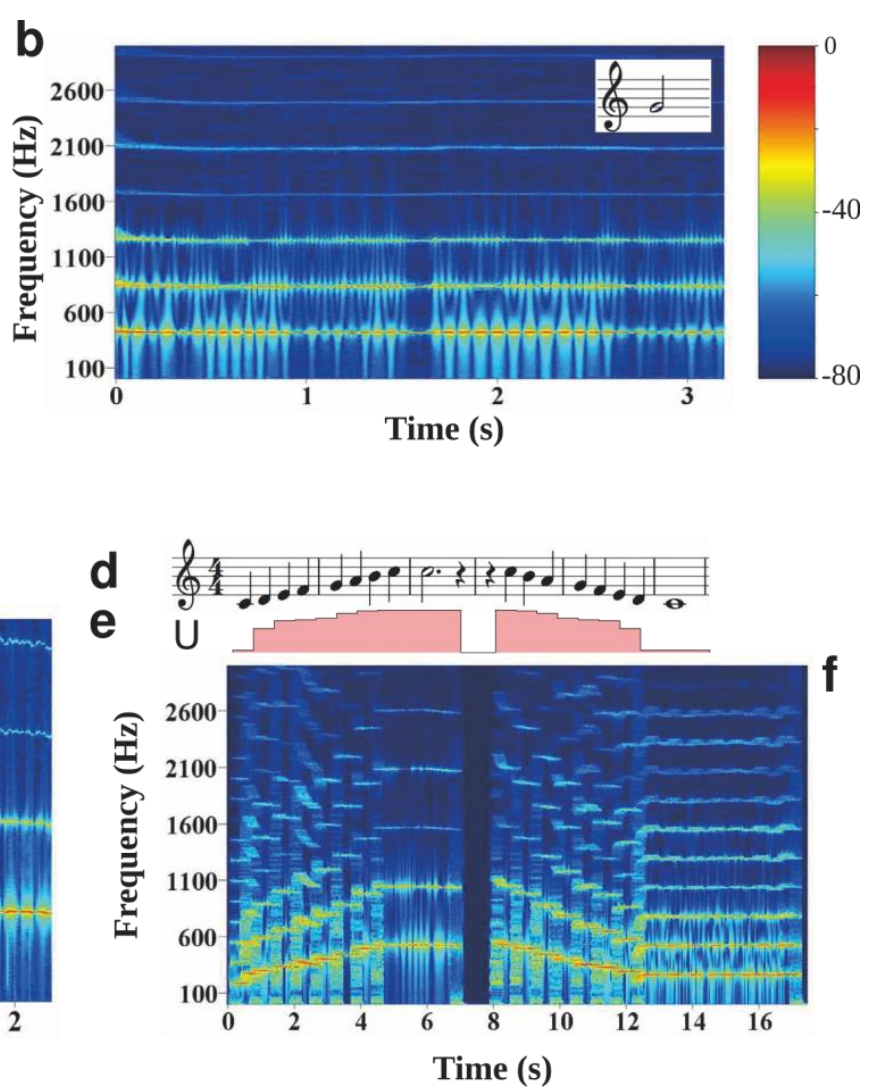

Figure 4.4: Generation of musical notes by the electrical control in droplet production. (a) $\mathrm{C}$ note produced at a stable frequency when $\mathrm{u}=0.05 \mathrm{~V}$. (b) $\mathrm{G}$ note produced at a stable frequency when $u=0.845 \mathrm{~V}$. (c) The detected frequencies when the voltage is varied. The volumetric flow rates are fixed in this case. (d,e) Illustration of the specific frequencies over the whole scale using different applied voltages. (f) The time trace of the frequency spectrum showing both the fundamental frequency and the harmonics of the signal.

We also investigate the stability of produced frequencies over a longer period of about $2000 \mathrm{~s}$ to determine the variability of the system (figure 4.5). We examine 3 different cases with and without the applied voltage. For the case of with the applied voltage, we use the musical note $A$ at droplet production frequencies of $440 \mathrm{~Hz}$ and $880 \mathrm{~Hz}$. Experimental results show that for the case of without the applied voltage, the variability in the measured frequency is about $3.8 \%$. With the applied voltage, the variability is $3.6 \%$ and $2.5 \%$ for the $440 \mathrm{~Hz}$ and $880 \mathrm{~Hz}$ case respectively. The 
observed variation in the frequencies with time is due to the mechanical stability of the syringe pump used to inject the fluids into the microfluidic devices. In order to circumvent this, the calibration of the frequencies and the applied voltages is run before the musical pieces are played to reduce the detuning due to this variation.

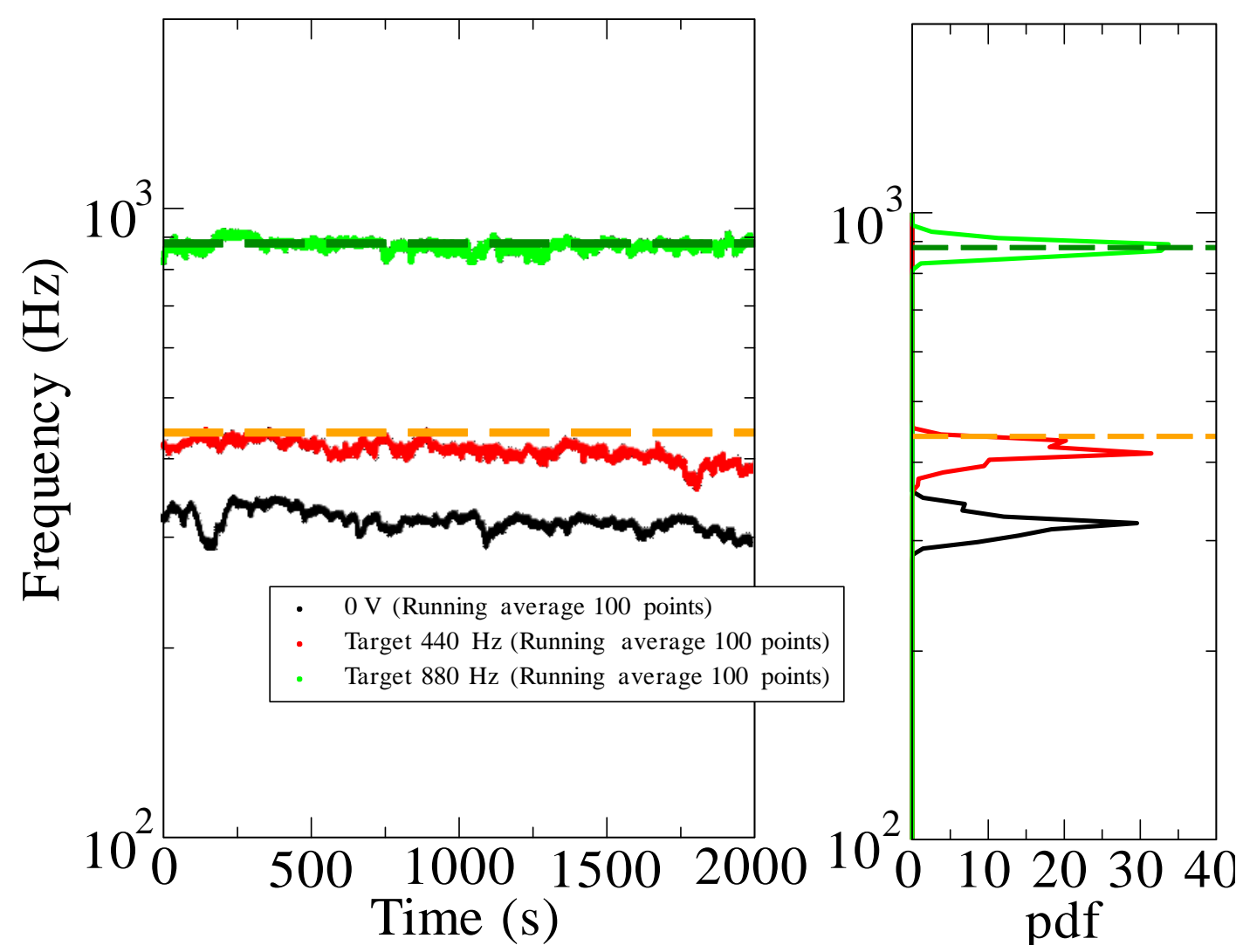

Figure 4.5: Stability of a note as a function of time. On the left, are the frequencies as a function of time. On the right, are the distributions of frequencies over the whole measurement window.

\subsubsection{Response time of the system}

We investigate here the response time of the system in order to determine the switching capabilities between different musical notes. An amplitude modulation (AM) signal is applied to the system at fixed volumetric flow rates. The AM signal is 
obtained by multiplying a $50 \mathrm{kHz}$ sinusoidal signal of $\mathrm{U}=500$ to $1040 \mathrm{~V}$ with a square wave signal. The frequency of the square wave signal varies between 40 to $400 \mathrm{~Hz}$. In order to determine the speed of the response, the AM signal is synchronize with the triggering of the high camera. This enables the video of the droplet formation process to be captured when the AM signal is applied. The videos are then analyzed with a simple image processing tool (Matlab, Mathworks) to plot the space time diagram of the droplet formation. The space time diagram is obtained by using a symmetry line in the center along the flow direction of the droplet. The black droplet interface appears as the white traces in the space time diagram due to the intersection with the symmetry line. In the diagrams, the slope of each line represents the speed of the droplets while the spacing between the lines represents the size of the droplets. Figure 4.6 represents the space time plots obtained at different flow rates and AM signals.

a

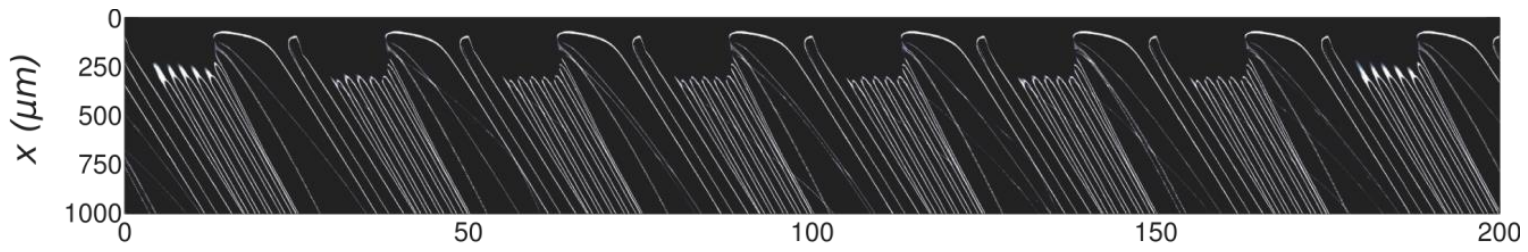

b

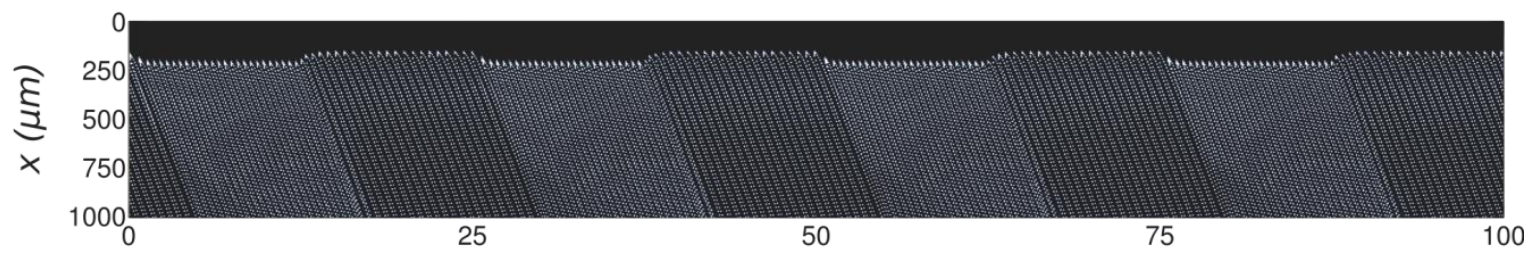

C

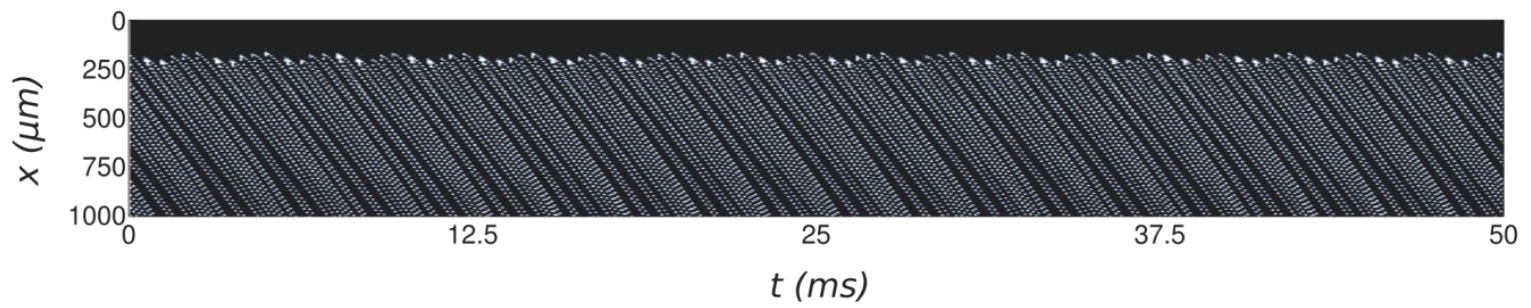

Figure 4.6: Space time diagrams obtained at various volumetric flow rates and AM signals. (a) When $Q_{d}=75 \mu \mathrm{l} / \mathrm{hr}$ and $Q_{c}=500 \mu \mathrm{l} / \mathrm{hr}$. The AM frequency is $40 \mathrm{~Hz}$ and the amplitude is $1000 \mathrm{~V}$. (b) When $Q_{d}=200 \mu \mathrm{l} / \mathrm{hr}$ and $Q_{c}=1600 \mu \mathrm{l} / \mathrm{hr}$. The AM frequency is $40 \mathrm{~Hz}$ and amplitude is $500 \mathrm{~V}$. (c) When $Q_{d}=200 \mu \mathrm{l} / \mathrm{hr}$ and $Q_{c}=1600$ $\mu \mathrm{l} / \mathrm{hr}$. The AM frequency is $400 \mathrm{~Hz}$ and amplitude is $500 \mathrm{~V}$. 
Experimental results shows that when the volumetric flow rates are fixed at $Q_{d}=75$ $\mu \mathrm{l} / \mathrm{hr}$ and $Q_{c}=500 \mu \mathrm{l} / \mathrm{hr}$ and $A M$ frequency is $40 \mathrm{~Hz}$, the system takes less than 25 $\mathrm{ms}$ to modulate the size of the droplets formed. This is represented by a change in the spacing between the lines indicating droplets of different volume. At fixed volumetric flow rate of $Q_{d}=75 \mu \mathrm{l} / \mathrm{hr}$, the frequency of the droplet generated can be related by $Q_{d}=f V$ using mass conservation. Here $f$ is the frequency of the generated droplet and $\mathrm{V}$ is the volume of the droplet. The equation indicates that at fixed volumetric flow rate of $Q_{d}$, a change in the size of the droplet $(V)$ will result in a proportionate change in the frequency in the droplet generated.

The space time diagrams also indicate that the process is highly repeatable and reliable as similar white traces of repeating patterns are observed in each of the tested parameter. We further test the response of the system by first increasing the volumetric flow rates and decreasing the modulation amplitude to $500 \mathrm{~V}$ (figure 4.6b). The AM frequency is fixed at $40 \mathrm{~Hz}$. This allows droplets to form at higher frequencies $(500 \mathrm{~Hz})$ and also avoiding the formation of the jetting regime. In this case, experimental results show that the response time of the system is less than $12.5 \mathrm{~ms}$ and the modulation of the droplet size is easily attained at $40 \mathrm{~Hz}$. In the last case, we increase the AM frequency to $400 \mathrm{~Hz}$ and also observed a modulation in the size of the droplets formed. Here we postulate that the response time of the system is at least in the order of $2.5 \mathrm{~ms}$ or higher.

We believe that the system is capable of obtaining faster response if experimental parameters such as the geometry of the channels and the fluids used are optimized. However, the fast response times obtained in the experiments is sufficient to switch the musical notes required to play the music.

\subsubsection{The Microfluidic Jukebox}

The electrical approach demonstrated above allows the fast switching of the droplet formation frequencies to play different notes in a musical sound track. However the approach does not create pauses between each different note due to the continuous flow of droplets. In order to distinguish between the notes, we generate a pause by 
switching off the laser through the use of electronic programming (Labview, National Instruments). This operation allows identical notes to be distinguished and also pauses to be created that is needed in musical sound tracks.

Figure 4.7 illustrates the instrumentation process of the microfluidic jukebox. The sound track of the songs are obtained from abc score (www.abcnotation.com). The scores are then transformed into a frequency vs time file via a home-made Perl program. The frequency sequence is then converted to the actuation voltage based on the tuning of the calibration which is run before the music is played and recorded. We also take into account the characteristic behavior of our system where the droplet frequency varies significantly during the transition from dripping to jetting. This transition in the droplet formation regime results in a frequency range which is not readily accessible. In this case, the Perl program adapts the musical piece to our microfluidic jukebox by shifting the frequency by a certain number of half tones until all the notes can be played. We finally use a Labview program to automatically set the required voltage and laser control in order to play the musical sound tracks.

We played two musical sound tracks with our microfluidic jukebox. For the first piece, Ode to Joy, the required frequencies are in the range of 170 to $340 \mathrm{~Hz}$. The musical notes are reliably reached and played with an error of less than $5 \%$. In order to test the limit of our microfluidic jukebox, we played a more demanding musical sound track namely Flight of the bumblebee which require faster switching between the notes and sharp transition over a larger range of frequencies. In this case, the microfluidic jukebox also follows the scores but a larger error is observed. Figure 4.8 depicts the comparison between the target frequency and the measured frequency for both the cases. 
a

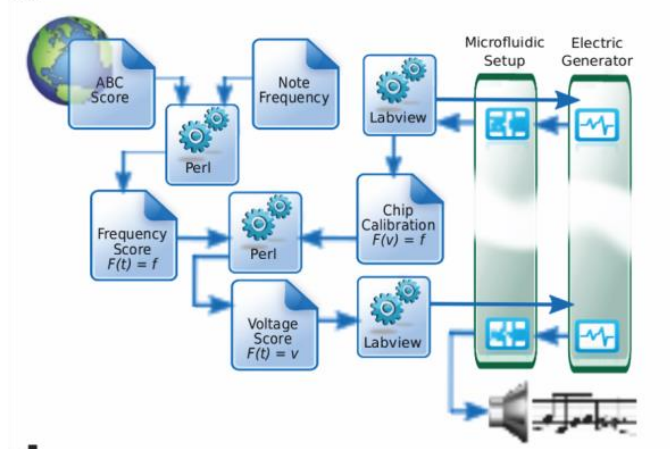

d

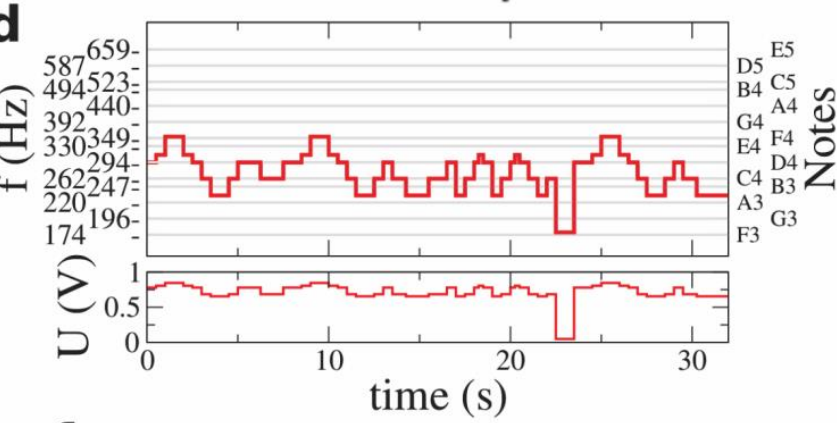

b
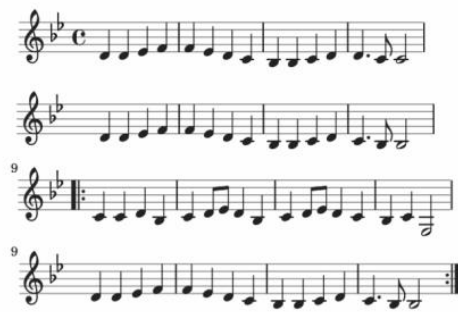

C

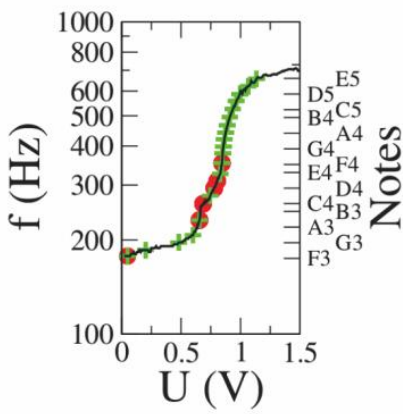

$\mathbf{f}$

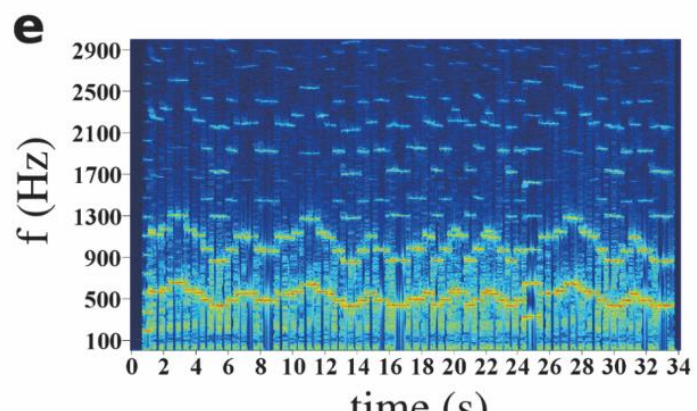
time (s)
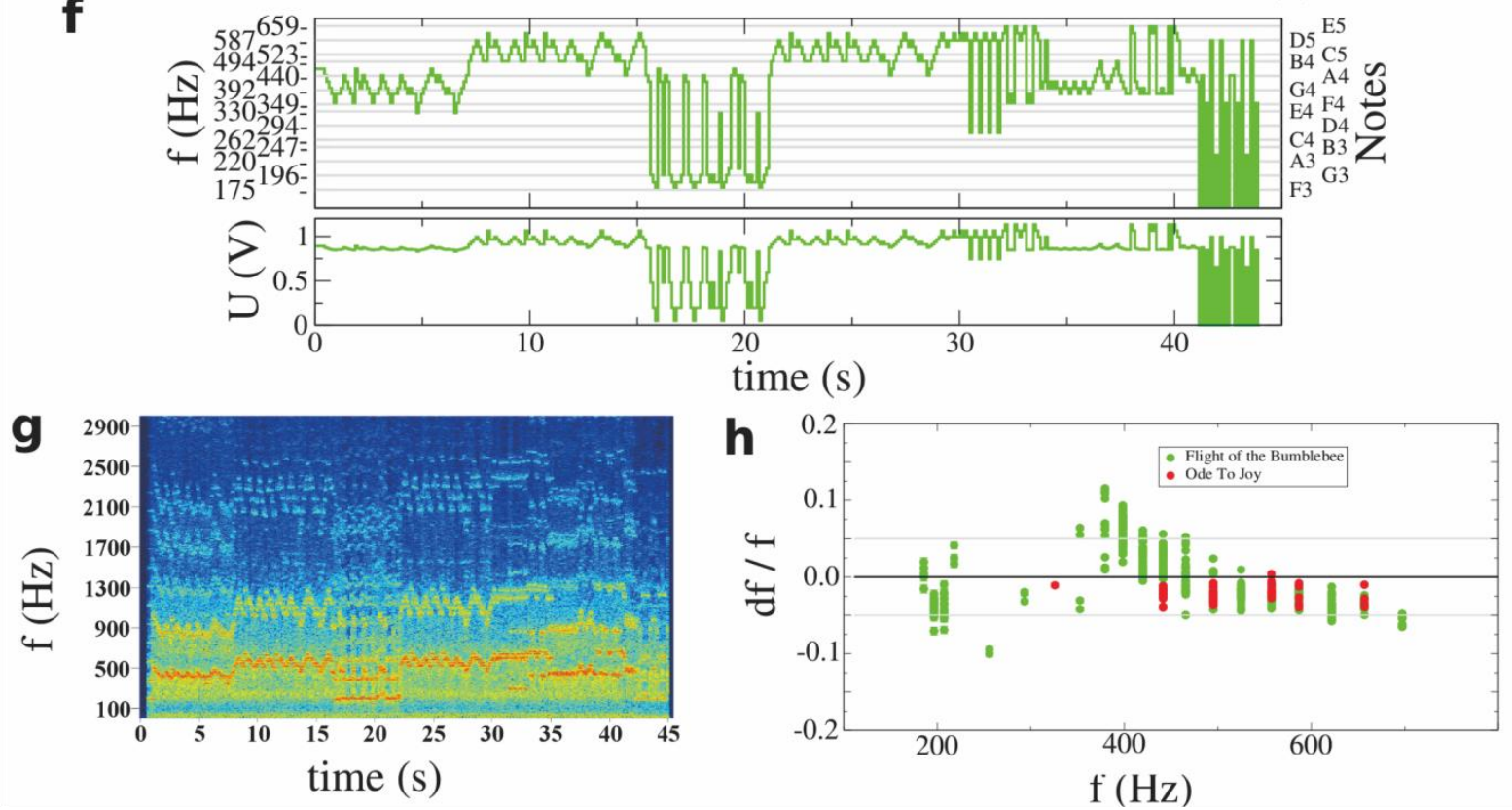

Figure 4.7: The Microfluidic Jukebox. (a) Illustration of the instrumentation process. (b) Musical scores of the piece Ode to Joy. (c) Calibration curve of the microfluidic device. (d) The conversion of the scores to the frequencies and actuation voltage required for the piece via the Perl program. (e) The signal are recorded and analyzed to determine the frequency spectrum of the sound as a function of time. (f) Recording of the voltage and frequencies for analysis. ( $g$ and $f$ ) Analyses done on the measured frequencies as a function of the target frequencies. 
(a)

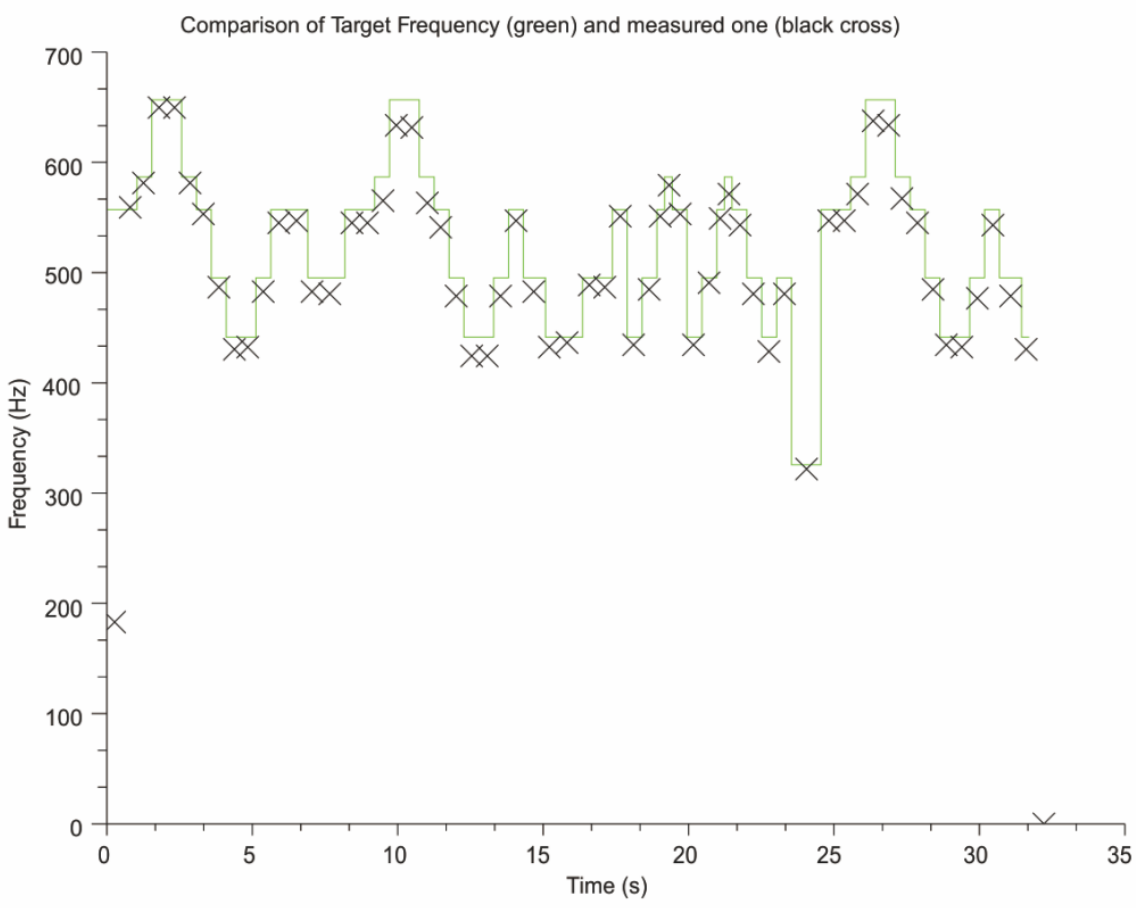

(b)

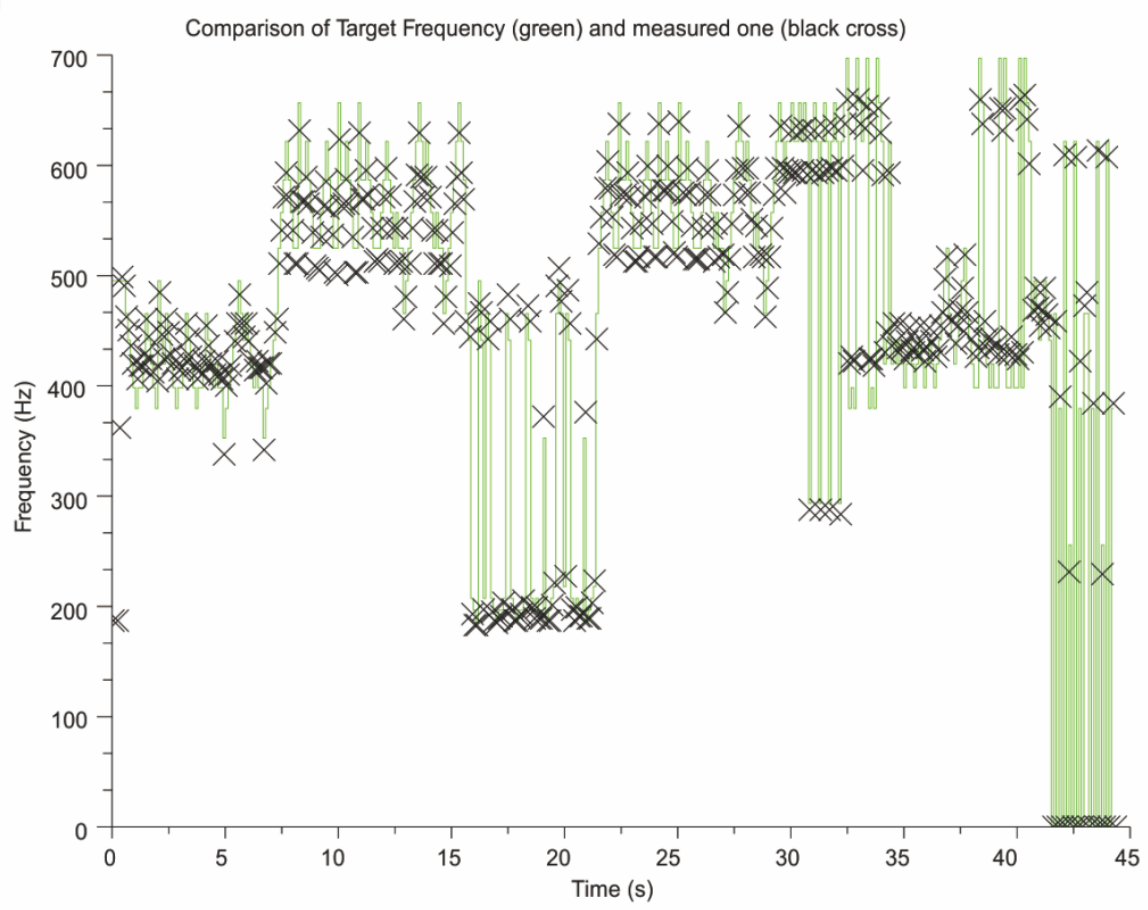

Figure 4.8: Accuracy in playing the targeted notes. (a) Ode to Joy and (b) Flight of the Bumblebee. 


\subsection{Discussion}

The microfluidic jukebox demonstrated here provides means to hear the sound produced by trains of micro-droplets. Another possible application is the use of it as a tool to determine the monodispersity in the generated droplet trains. While conventional method uses imaging processing to measure the size of the droplets, one could effectively use this new technology to instantly hear if the production of the droplets is changing over time.

Our system is also reminiscent of the bubble piano developed by Minnaert [99]. He produces musical songs by generating bubbles of controlled size and then uses the resonance at different sizes to produce musical notes. In contrast to his system, our approach does not rely on the resonance of the bubble size to produce musical notes but rather the instabilities of a liquid jet due to an AC voltage induced electric field. We do not use the pressure wave in the system to produce music sound tracks. In our system, a transduced signal of the corresponding droplet formation frequency is used instead.

\subsection{Conclusion}

In conclusion, we demonstrate a musical interpretation of droplet based microfluidic using the technology developed in the previous chapters. The use of the AC voltage induced electric field allows the droplets formation frequencies to modulate reliably at time scale of milli-seconds. This fast actuation in turn provides the means to produce musical sound tracks through the transduced frequencies.

${ }^{* * *}$ Movies and sound tracks related to this chapter can be obtained from the following supplementary material of the following link:

http://www.nature.com/srep/2014/140430/srep04787/full/srep04787.html 


\section{Part 4: Conclusion}

\section{Chapter 5: Conclusion and outlook}

\section{Content}

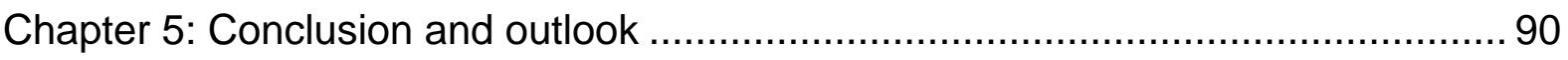

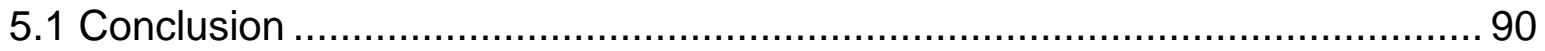

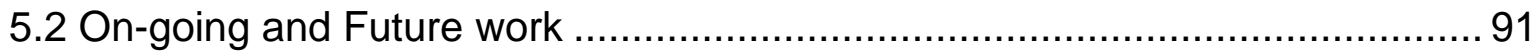




\section{Chapter 5: Conclusion and outlook}

\subsection{Conclusion}

The main objective of this thesis was to develop a tool to actively and reliably control the size of the droplet generated in a microfluidic device. This is achieved by using an $A C$ voltage induced electric field coupled in a microfluidic flow focusing device. In the propose method, the electrodes are not in contact with the fluids and no alignments of the electrodes with the microchannels are needed. This eliminates undesirable electrochemical effects such as electrolysis which occurs at high voltages and in turn affects the stability of the droplet generated. An additional level of convenience is also given to the end users as the devices can be fabricated easily.

We have also addressed several important issues in this thesis. In chapter 2, we present the conceptual results obtained and discuss on the improvements to both the designs and experimental works. These results lay the physical ground work needed for the subsequent chapter. In chapter 3 , we studied extensively on the influences of various important parameters using a simplified design. Experimental results obtained suggested the importance of both the applied sinusoidal signal frequencies and conductivities of the dispersed phase fluid. Depending on the configuration of the electrodes, the voltage divider rule can be applied to obtain an electrical equivalent model to calculate the voltage at the tip of the dispersed phase fluid. We found that the system behaves akin to a high pass filter where the ratio of $\mathrm{f} / \mathrm{k}$ is the sole parameter influencing the phenomenon. Below the cut-off, the voltage at the tip reduces to only about $25 \%$ and results in a transition from the unstable jetting to the dripping regime. The calculated value agrees well with the phase diagrams presented in all the 3 different configurations. However, we are still unable to explain the transition from the stable to the unstable jetting regime which occurs at various frequencies and conductivities.

We also present an electrohydrodynamic model to account for both the hydrodynamic and electrical effect. The proposed effective capillary number takes into consideration the effect of the Maxwell stress which acts in the opposite normal 
direction to the Laplace pressure. Power laws describing the scaling of the diameters of the droplets in both regimes are also presented. A weak dependence is observed when the droplets are formed in the jetting regime. The proposed model also does not dictate the transition from the dripping to the jetting regime.

In chapter 4, we demonstrate the both the robustness and reliability of the concept by producing musical sound tracks using the microfluidic jukebox. The response time of the system is also investigated using different AM modulation signals at different volumetric flow rates. Experimental results show that the system is able to respond to the electrical actuation at time scale as fast as $2.5 \mathrm{~ms}$. We played two very different musical pieces namely Ode to Joy and Flight of the bumblebee using the microfluidic jukebox. Slight variations between the target frequencies and the measured frequencies are obtained for both pieces.

In summary, we have shown that this method of active control is a convenient and versatile to control the size or frequencies of the droplet generated in a microfluidic device. The additional level of control provided by this method is of potential interest for high throughput droplet based applications.

\subsection{On-going and Future work}

At this point, some elements of this work are still under investigation and various collaborative projects have been formed with different groups. The study of influences of the viscosity ratio between the fluids in this system (Asst Prof Elena Castro-Hernández, Universidad de Sevilla), the transition between the stable jetting to the unstable jetting using numerical simulations (Prof Alfonso M Gañán-Calvo, Universidad de Sevilla) and the influence of different liquid crystals (Dr Venkata Subba Rao Jampani, MPI-DS). Experimental works have also been completed for the study of mineral oil without the use of surfactants and the analysis is still in progress. This work could be extended in various directions, a comparison with the incorporation of the orifice as shown in chapter 2, the formation of Taylor's cone to produce submicron droplets and the effect in different geometrical configurations. 


\section{Part 5: Appendices}

\section{Appendices}

\section{Content}

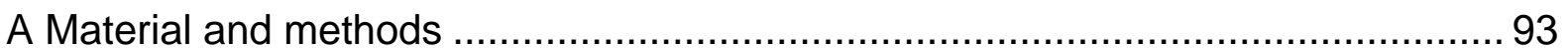

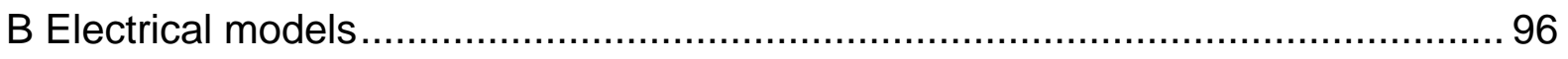

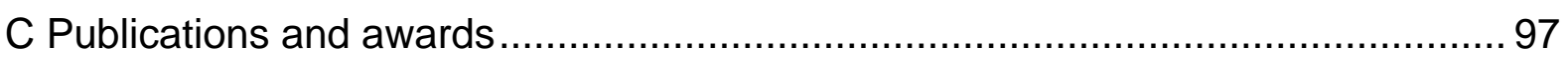

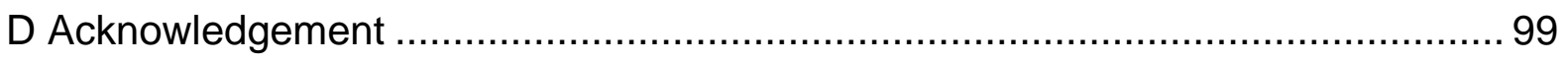

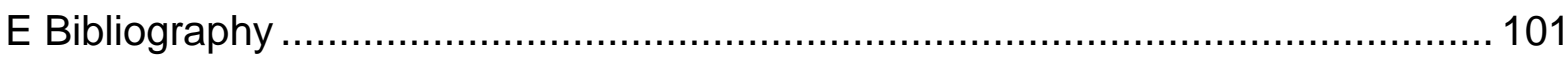

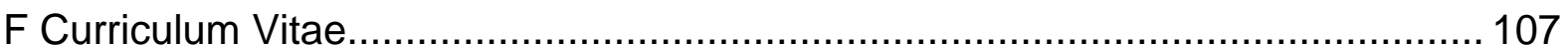




\section{A Material and methods}

This section describes the material and methods used in the course of this thesis.

\section{Continuous phase fluid}

Mineral oil (M5904, Sigma Aldrich) with 5\% wt/wt of non-ionic surfactant (SPAN 80, Sigma Aldrich) is used as the continuous phase fluid. The fluid is prepared by mixing $1.895 \mathrm{~g}$ of SPAN 80 in $36 \mathrm{~g}$ of mineral oil.

\section{Dispersed phase fluid}

The dispersed phase fluid used is milli-Q water or milli-Q water mixed with either $\mathrm{NaCl}$ salt or $\mathrm{HCl}$ solutions by weight in concentrations stated in the text. The mixture with the highest concentration is first prepared and the remaining concentrations are obtained by standard dilution method.

\section{Microfluidic devices}

The microfluidic devices are replicated from SU-8 masters and bonded to ITO glasses (CG-60IN-S215, Delta Technologies) using standard air plasma. The devices are flushed with Aquapel solutions before use. The fabrication process of both the SU-8 masters and PDMS are shown below.

\section{SU-8 Masters}

The following recipe is used for the making the master mold.

1. Clean the silicon wafer repeatedly with Isopropanol and Acetone solutions.

2. Blows dry the wafer with nitrogen gas.

3. Place the wafer at a $200^{\circ} \mathrm{C}$ hot plate for 20 minutes for dehydration baking.

4. Remove the wafer and allow cooling to room temperature.

5. Spin coat SU-8 3025 at about $2100 \mathrm{rpm}$ to achieve a thickness of $35 \mu \mathrm{m}$.

6. Place the wafer for soft baking at $65^{\circ} \mathrm{C}$ for 3 minutes, $95^{\circ} \mathrm{C}$ for 12 minutes and then $65^{\circ} \mathrm{C}$ again for 1 minute. This step cooling reduces the thermal stress. 
7. Remove the wafer for cooling for about $\mathbf{3 0}$ minutes.

8. Clean the photo-mask with Isopropanol and dry with nitrogen gas.

9. Expose the wafer with the photo-mask using the EVG exposure unit. Use hard contact mode for about 15 seconds exposure time.

10. Place the wafer for post exposure baking (PEB) at $65^{\circ} \mathrm{C}$ for 1 minute, $95^{\circ} \mathrm{C}$ for 4 minutes and then $65^{\circ} \mathrm{C}$ again for 1 minute.

11. Develop wafer for about 10 minutes with occasional shaking.

12. Check by cleaning with Isopropanol solution. A white residue will form if the development is not complete.

13. After complete development, clean with Isopropanol solution and dry wafer with nitrogen gas.

14. Hard bake at $150^{\circ} \mathrm{C}$ for 2 minutes.

15. Measure the depth of the channels at various locations.

\section{Replication with PDMS}

1. PDMS (Sylgard 184 elastomer kit, Dow Corning) is mixed with the cross linker in the ratio of $10: 1$.

2. The mixture is degassed under vacuum for 1 hour until all the air bubbles are removed and the mixture becomes clear.

3. The SU-8 master is clean with Isopropanol and dried with nitrogen gas.

4. The PDMS mixture is poured over the SU-8 master and cure at $85^{\circ} \mathrm{C}$ for 2 hours. Bubbles induced during the pouring are remove by gently blowing over the surface of the mixture.

5. After curing, the replicated designs are cut and peeled off from the master mold. Fluidic access holes are punched with a $0.75 \mathrm{~mm}$ Harris puncher.

6. The PDMS devices and ITO glasses are cleaned with Isopropanol and Milli-Q water.

7. Dry first with nitrogen gas and then keep in at $85^{\circ} \mathrm{C}$ oven for 1 hour. This is to ensure that no residue fluids remain on the devices.

8. Remove and bond with air plasma for 35 seconds.

9. Place in $85{ }^{\circ} \mathrm{C}$ oven for another hour to improve the bonding. 


\section{Electrodes}

1. Place the bonded microfluidic devices on a hotplate at about $70{ }^{\circ} \mathrm{C}$ which is slightly higher than the melting point of the alloy.

2. Insert the alloy into the fluidic access holes slowly.

3. Insert the wire connections.

4. Secure the wire by placing UV glue around the wire.

5. Cure the UV glue under a UV lamp for 10 minutes.

\section{Surface modification}

1. Flush the microfluidic channels with nitrogen gas.

2. Flush with Aquapel solutions and leave it there for a few minutes.

3. Flush away the aquapel using nitrogen gas.

\section{ITO Electrode}

1. Clean the ITO glass with Isopropanol and Acetone solutions.

2. Blows dry with nitrogen gas.

3. Place the ITO glass at a $200{ }^{\circ} \mathrm{C}$ hot plate for 20 minutes for dehydration baking.

4. Spin coat HPR 504 at about $3000 \mathrm{rpm}$ to achieve a thickness of $1.6 \mu \mathrm{m}$.

5. Soft bake at $100^{\circ} \mathrm{C}$ for 6 mins.

6. Remove the ITO glass plate from the hotplate and let it cool to room temperature.

7. Expose using EVG Mask Aligner for 5s using hard contact.

8. Develop with HPRD-429 for 1 minute.

9. Post exposure baking at $100^{\circ} \mathrm{C}$ for 6 minutes.

10. Etch away the exposed ITO using $\mathrm{HCl}: \mathrm{H}_{2} \mathrm{O}: \mathrm{HNO}_{3}=4: 2: 1$ mixture for 20 minutes.

11. Immerse the ITO glass plate into Microstrip 2001 for 3 minutes to remove the photoresist.

12. Inspect under a microscope. 


\section{B Electrical models}

The voltage divider rule is used to derive the expressions for the different electrode configurations. A typical example for electrode in configuration $A$ is shown below:

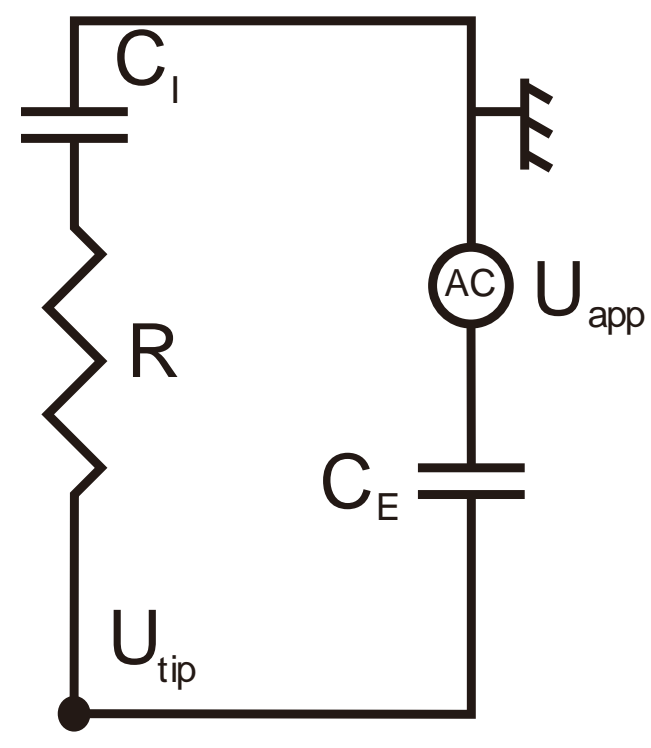

For an AC source, we consider the impedance of each component. Hence using the voltage divider rule, the following expression can be derived:

$$
U_{t i p}=U_{a p p}\left(\frac{Z_{F}+Z_{I}}{Z_{F}+Z_{I}+Z_{E}}\right)
$$

In this configuration, the bottom pair of the electrodes is grounded. Hence, the potential difference is $U_{\text {tip }} \approx U_{\text {tip }}-0$. Similar expressions and methods can be obtained for all the different electrode configurations. 


\section{Publications and awards}

Publications

1. S. H. Tan, F. Maes, B. Semin, J. Vrignon, and J.-C. Baret, "The Microfluidic Jukebox," Sci. Rep., vol. 4, 2014.

2. S. H. Tan, B. Semin, and J.-C. Baret, "Microfluidic flow-focusing in ac electric fields," Lab on a Chip, vol. 14, pp. 1099-1106, 2014.

3. S. H. Tan, B. Semin, and J.-C. Baret, "Electrohydrodynamic in microfluidic flow focusing," In preparation.

4. V. S. R. Jampani, K. Peddireddy, S. H. Tan, J. C. Baret, S. Herminghaus and C. H. Bahr, "Aqueous droplet production in nematic flows under AC fields," In preparation.

Proceedings

1. S. H. Tan, B. Semin, F. Maes, and J.-C. Baret, "Electric control in dropletbased microfluidic," Proceedings of MicroTas 2012.

2. S. H. Tan, B. Semin, and J.-C. Baret, "Novel Electrical Control in Droplet Microfluidics Using an AC Electric Field," Proceedings of ASME 2013.

3. V. S. R. Jampani, K. Peddireddy, S. H. Tan, J. C. Baret, S. Herminghaus and C. H. Bahr, "Microfluidic droplet production in a nematic matrix under AC electric fields," Proceedings of 41st German Conference on Liquid Crystals, Magdeburg, Germany.

Awards

1. Max Planck International research scholarship 2010.

2. Tan Kah Kee Young Inventor's Award 2014. Merit Prize.

3. Tan Kah Kee Postgraduate Scholarship 2012. 
Patent

1. S. H. Tan, B. Semin, and J.-C. Baret, "Method and fluidic microsystem for generating droplets dispersed in a continuous phase," Patent number: WO2013143562. Publication date: 2013/10/3.

Talks

1. Ecole Normale Superieure (ENS), Paris, France, 2012.

2. ASME conference, Sapporo, Japan, 2012.

3. Nanyang Technological University (NTU), Singapore, 2013.

Posters

1. MicroTas, Okinawa, Japan, 2012. 


\section{Acknowledgement}

I would like to express my deepest gratitude to Prof. Dr. Jean-Christophe Baret for his kind supervision, relentless patient and utmost guidance throughout the course of my doctorate studies. He has provided me the freedom to pursue my interest without any limitation or restriction of any kind. Often, his encouragement and passion for science is the source of my inspiration. During my years with him, the list of what he has taught me is exhaustive. I would say, he has not only educated me with all the necessary tools for research but have also shown me many important things in life. I am very fortunate to have met him and will also be forever grateful to have him as my advisor and my mentor. Thank you! Merci!

My thesis committee members, Prof. Dr. Joerg Enderlein and Prof. Dr. Stephan Herminghaus have provided me with invaluable advice and suggestions during the yearly meetings. Often, their feedbacks are innovative and timely which greatly help the progress of my work. Their insightful comments have also ascertained the quality of my work and also serve as a great form of motivation. This thesis will not have been possible without the guidance from both of you.

Dr. Benoit Semin has played an immerse role in my doctorate journey. He has played the role of a brother, a sibling and a family member. At work, he taught me physics and helped me in all my programming needs. Often, discussing with him helped me to understand a lot of the important findings in my research. Outside of work, he will often invite me over for dinner and spend quality time with me making me feel at home. He is not only good in science but a great cook as well. His culinary skills are one of the best that I have tasted so far. When he left for his new position in Paris, he still takes the effort to have weekly phone discussions with me. Without him, this thesis will not have been possible!

I would also like to thank both Dr. Florine Maes and Jeremy Vrignon for their part in the microfluidic jukebox. Their musical talents allow the work to be performed with great joy and fun. It must be hard for them to have to explain all the basic musical concepts to me which are needed for this work to be such a success. Last but not least, congratulation to both of you on your new-born! 
I would also like to thank both Prof Alfonso M Gañán-Calvo and Ast Prof Elena Castro-Hernández from the Universidad de Sevilla for their help in the collaborative projects. This collaboration provides greater insights to the current work.

I am also very fortunate to have Prof. Dr. Nguyen Nam Trung as my first academic mentor in research. It was him whom introduced me to the field of droplet microfluidics and shown me how interesting it is. Throughout the years, his fatherly figure provided me with endless streams of motivation, support, encouragement and advice. At times of despair, he will always be there for me to lean on as a supporting pillar. At good times, he is often more happy than me and proud of what I have achieved. His role is essential and vital to my life long journey in the academia.

My journey in Germany will not be as pleasant without the help of Monika Teuteberg, Thomas Eggers, Markus Benderoth, Udo Kraft, Wolf Keiderling, Guido Schriever and Sibylle Nägle in both administrative and personal matters. Living alone in a foreign land is not easy but their kind hospitality and willingness to help out made my stay relatively easier to cope.

I would also like to thank the support of GGNB doctorate school and all the administrative staffs for their hard work - Antje Erdmann and Frauke Bergmann. Their kind help in all the administrative matters make the doctorate journey pleasant and enjoyable.

I have met many great colleagues, office mates, roommates and friends during my time in the institute. Their presence allows me to adapt to the life in Germany and made me feel like I am not alone despite being so far away from my home - Xunda, Bao Fang, Shi Liang, Song Chuan, Markus Helmer, Kumar, Ciro, Paul Steffan, Frank, Daniel Herde, Christoph Gögelein, Kristian Hanke, Jann Ohle Claussen, Julie, Marta, Eric Stellamanns, Sonia, Martin Brinkmann, Zrinka Gattin, Thomas Hiller, Nirmal, Philipp, Birte, Jieseok, Renaud Dufour, Ingmar Polenz, Zakaria, Elena and Xiao Peng. Lastly, this thesis will not be possible without the support of my family members. My mum and dad whom have been always there and supported me in whatever ways they could. My wife who has been by my side, accompanying me and providing all the mental support, encouragement while I tried to complete the writing of my thesis. Without you all, I would not have gotten so far. Thank you! Merci! Danke! 


\section{E Bibliography}

[1] A. Huebner, S. Sharma, M. Srisa-Art, F. Hollfelder, J. B. Edel, and A. J. deMello, "Microdroplets: A sea of applications?," Lab on a Chip, vol. 8, pp. 1244-1254, 2008.

[2] S. Haeberle and R. Zengerle, "Microfluidic platforms for lab-on-a-chip applications," Lab on a Chip, vol. 7, pp. 1094-1110, 2007.

[3] H. Song, D. L. Chen, and R. F. Ismagilov, "Reactions in Droplets in Microfluidic Channels," Angewandte Chemie International Edition, vol. 45, pp. 7336-7356, 2006.

[4] H. N. Joensson and H. Andersson Svahn, "Droplet Microfluidics-A Tool for Single-Cell Analysis," Angewandte Chemie International Edition, vol. 51, pp. 12176-12192, 2012.

[5] P. S. Dittrich and A. Manz, "Lab-on-a-chip: microfluidics in drug discovery," Nat Rev Drug Discov, vol. 5, pp. 210-218, 2006.

[6] J. R. Millman, K. H. Bhatt, B. G. Prevo, and O. D. Velev, "Anisotropic particle synthesis in dielectrophoretically controlled microdroplet reactors," Nat Mater, vol. 4, pp. 98-102, 2005.

[7] O. Skurtys and J. M. Aguilera, "Applications of Microfluidic Devices in Food Engineering," Food Biophysics, vol. 3, pp. 1-15, 2008/03/01 2008.

[8] M. T. Guo, A. Rotem, J. A. Heyman, and D. A. Weitz, "Droplet microfluidics for high-throughput biological assays," Lab on a Chip, vol. 12, pp. 2146-2155, 2012.

[9] Y. Zhu and B. Power, "Lab-on-a-chip in Vitro Compartmentalization Technologies for Protein Studies," in Protein - Protein Interaction. vol. 110, M. Werther and H. Seitz, Eds., ed: Springer Berlin Heidelberg, 2008, pp. 81-114.

[10] Y. Zhang and P. Ozdemir, "Microfluidic DNA amplification-A review," Analytica Chimica Acta, vol. 638, pp. 115-125, 2009.

[11] T. Ward, M. Faivre, M. Abkarian, and H. A. Stone, "Microfluidic flow focusing: Drop size and scaling in pressure versus flow-rate-driven pumping," ELECTROPHORESIS, vol. 26, pp. 3716-3724, 2005.

[12] J.-C. Baret, O. J. Miller, V. Taly, M. Ryckelynck, A. El-Harrak, L. Frenz, C. Rick, M. L. Samuels, J. B. Hutchison, J. J. Agresti, D. R. Link, D. A. Weitz, and A. D. Griffiths, "Fluorescence-activated droplet sorting (FADS): efficient microfluidic cell sorting based on enzymatic activity," Lab on a Chip, vol. 9, pp. 1850-1858, 2009.

[13] D. Pekin, Y. Skhiri, J.-C. Baret, D. Le Corre, L. Mazutis, C. Ben Salem, F. Millot, A. El Harrak, J. B. Hutchison, J. W. Larson, D. R. Link, P. Laurent-Puig, A. D. Griffiths, and V. Taly, "Quantitative and sensitive detection of rare mutations using droplet-based microfluidics," Lab on a Chip, vol. 11, pp. 21562166, 2011.

[14] T. Nisisako, T. Torii, and T. Higuchi, "Droplet formation in a microchannel network," Lab on a Chip, vol. 2, pp. 24-26, 2002.

[15] T. Thorsen, R. W. Roberts, F. H. Arnold, and S. R. Quake, "Dynamic Pattern Formation in a Vesicle-Generating Microfluidic Device," Physical Review Letters, vol. 86, pp. 4163-4166, 2001. 
[16] S. L. Anna, N. Bontoux, and H. A. Stone, "Formation of dispersions using "flow focusing" in microchannels," Applied Physics Letters, vol. 82, pp. 364366, 2003.

[17] S. L. Anna and H. C. Mayer, "Microscale tipstreaming in a microfluidic flow focusing device," Physics of Fluids (1994-present), vol. 18, pp. -, 2006.

[18] R. Dreyfus, P. Tabeling, and H. Willaime, "Ordered and Disordered Patterns in Two-Phase Flows in Microchannels," Physical Review Letters, vol. 90, p. 144505, 2003.

[19] Y. Hong and F. Wang, "Flow rate effect on droplet control in a co-flowing microfluidic device," Microfluidics and Nanofluidics, vol. 3, pp. 341-346, 2007/06/01 2007.

[20] R. Suryo and O. A. Basaran, "Tip streaming from a liquid drop forming from a tube in a co-flowing outer fluid," Physics of Fluids (1994-present), vol. 18, pp. -, 2006.

[21] C. Cramer, P. Fischer, and E. J. Windhab, "Drop formation in a co-flowing ambient fluid," Chemical Engineering Science, vol. 59, pp. 3045-3058, 2004.

[22] L. Shui, F. Mugele, A. van den Berg, and J. C. T. Eijkel, "Geometry-controlled droplet generation in head-on microfluidic devices," Applied Physics Letters, vol. 93, pp. -, 2008.

[23] L. Shui, A. van den Berg, and J. C. T. Eijkel, "Capillary instability, squeezing, and shearing in head-on microfluidic devices," Journal of Applied Physics, vol. 106, pp. -, 2009.

[24] C. N. Baroud, F. Gallaire, and R. Dangla, "Dynamics of microfluidic droplets," Lab on a Chip, vol. 10, pp. 2032-2045, 2010.

[25] G. F. Christopher and S. L. Anna, "Microfluidic methods for generating continuous droplet streams," Journal of Physics D: Applied Physics, vol. 40, p. R319, 2007.

[26] R. Seemann, M. Brinkmann, T. Pfohl, and S. Herminghaus, "Droplet based microfluidics," Reports on Progress in Physics, vol. 75, p. 016601, 2012.

[27] T. T. Al-Housseiny, P. A. Tsai, and H. A. Stone, "Control of interfacial instabilities using flow geometry," Nat Phys, vol. 8, pp. 747-750, 2012.

[28] M. L. J. Steegmans, A. Warmerdam, K. G. P. H. Schroën, and R. M. Boom, "Dynamic Interfacial Tension Measurements with Microfluidic Y-Junctions," Langmuir, vol. 25, pp. 9751-9758, 2009/09/01 2009.

[29] J. D. Tice, A. D. Lyon, and R. F. Ismagilov, "Effects of viscosity on droplet formation and mixing in microfluidic channels," Analytica Chimica Acta, vol. 507, pp. 73-77, 2004.

[30] C. N. Baroud, M. Robert de Saint Vincent, and J.-P. Delville, "An optical toolbox for total control of droplet microfluidics," Lab on a Chip, vol. 7, pp. 1029-1033, 2007.

[31] S. M. S. Murshed, S. H. Tan, and N.-T. Nguyen, "Temperature dependence of interfacial properties and viscosity of nanofluids for droplet-based microfluidics," Journal of Physics D: Applied Physics, vol. 41, p. 085502, 2008.

[32] N.-T. Nguyen, T.H. Ting, Y.F. Yap, T.N. Wong, J. C.K. Chai, W.L. Ong, J. Zhou, S. H. Tan and L. Yobas, "Thermally mediated droplet formation in microchannels," Applied Physics Letters, vol. 91, pp. -, 2007.

[33] S. H. Tan, S. M. S. Murshed, N.-T. Nguyen, T.N. Wong, and L. Yobas, "Thermally controlled droplet formation in flow focusing geometry: formation regimes and effect of nanoparticle suspension," Journal of Physics D: Applied Physics, vol. 41, p. 165501, 2008. 
[34] C. A. Stan, S. K. Y. Tang, and G. M. Whitesides, "Independent Control of Drop Size and Velocity in Microfluidic Flow-Focusing Generators Using Variable Temperature and Flow Rate," Analytical Chemistry, vol. 81, pp. 2399-2402, 2009/03/15 2009.

[35] A. R. Abate, M. B. Romanowsky, J. J. Agresti, and D. A. Weitz, "Valve-based flow focusing for drop formation," Applied Physics Letters, vol. 94, pp. -, 2009.

[36] H. Suz-Kai, C. Cheng-Tso, and L. Gwo-Bin, "Micro-droplet formation utilizing microfluidic flow focusing and controllable moving-wall chopping techniques," Journal of Micromechanics and Microengineering, vol. 16, p. 2403, 2006.

[37] H. Willaime, V. Barbier, L. Kloul, S. Maine, and P. Tabeling, "Arnold Tongues in a Microfluidic Drop Emitter," Physical Review Letters, vol. 96, p. 054501, 2006.

[38] A. Bransky, N. Korin, M. Khoury, and S. Levenberg, "A microfluidic droplet generator based on a piezoelectric actuator," Lab on a Chip, vol. 9, pp. 516520, 2009.

[39] S. H. Tan, N.-T. Nguyen, L. Yobas, and T. G Kang, "Formation and manipulation of ferrofluid droplets at a microfluidic $T$-junction," Journal of Micromechanics and Microengineering, vol. 20, p. 045004, 2010.

[40] S. H. Tan and N.-T. Nguyen, "Generation and manipulation of monodispersed ferrofluid emulsions: The effect of a uniform magnetic field in flow-focusing and T-junction configurations," Physical Review E, vol. 84, p. 036317, 2011.

[41] Y. Wu, T. Fu, Y. Ma, and H. Z. Li, "Ferrofluid droplet formation and breakup dynamics in a microfluidic flow-focusing device," Soft Matter, vol. 9, pp. 97929798, 2013.

[42] M. Florent, A. V. Siva, G. Hao, E. Dirk van den, and M. Frieder, "Electrowetting-controlled droplet generation in a microfluidic flow-focusing device," Journal of Physics: Condensed Matter, vol. 19, p. 462101, 2007.

[43] H. Gu, M. H. G. Duits, and F. Mugele, "A hybrid microfluidic chip with electrowetting functionality using ultraviolet (UV)-curable polymer," Lab on a Chip, vol. 10, pp. 1550-1556, 2010.

[44] H. Gu, F. Malloggi, S. A. Vanapalli, and F. Mugele, "Electrowetting-enhanced microfluidic device for drop generation," Applied Physics Letters, vol. 93, pp. -, 2008.

[45] H. Gu, C. U. Murade, M. H. G. Duits, and F. Mugele, "A microfluidic platform for on-demand formation and merging of microdroplets using electric control," Biomicrofluidics, vol. 5, pp. -, 2011.

[46] P. He, H. Kim, D. Luo, M. Marquez, and Z. Cheng, "Low-frequency ac electroflow-focusing microfluidic emulsification," Applied Physics Letters, vol. 96, pp. $-, 2010$.

[47] H. Kim, D. Luo, D. Link, D. A. Weitz, M. Marquez, and Z. Cheng, "Controlled production of emulsion drops using an electric field in a flow-focusing microfluidic device," Applied Physics Letters, vol. 91, pp. -, 2007.

[48] D. R. Link, E. Grasland-Mongrain, A. Duri, F. Sarrazin, Z. Cheng, G. Cristobal, M. Marquez, and D. A. Weitz, "Electric Control of Droplets in Microfluidic Devices," Angewandte Chemie International Edition, vol. 45, pp. 2556-2560, 2006.

[49] F. Malloggi, H. Gu, A. G. Banpurkar, S. A. Vanapalli, and F. Mugele, "Electrowetting --A versatile tool for controlling microdrop generation," The European Physical Journal E, vol. 26, pp. 91-96, 2008/05/01 2008. 
[50] Y. N. Cheung and H. Qiu, "Acoustic microstreaming for droplet breakup in a microflow-focusing device," Applied Physics Letters, vol. 97, pp. -, 2010.

[51] L. Schmid and T. Franke, "SAW-controlled drop size for flow focusing," Lab on a Chip, vol. 13, pp. 1691-1694, 2013.

[52] L. Schmid and T. Franke, "Acoustic modulation of droplet size in a T-junction," Applied Physics Letters, vol. 104, pp. -, 2014.

[53] A. Diguet, H. Li, N. Queyriaux, Y. Chen, and D. Baigl, "Photoreversible fragmentation of a liquid interface for micro-droplet generation by light actuation," Lab on a Chip, vol. 11, pp. 2666-2669, 2011.

[54] S. H. Tan, B. Semin, F. Maes, and J.-C. Baret, "ELECTRIC CONTROL IN DROPLET-BASED MICROFLUIDICS," MicroTas 2012.

[55] S. H. Tan, S. M. S. Murshed, N.-T. Nguyen, T. N. Wong, and L. Yobas, "Thermally controlled droplet formation in flow focusing geometry: formation regimes and effect of nanoparticle suspension," Journal of Physics D: Applied Physics, vol. 41, p. 165501, 2008.

[56] O. Ozen, N. Aubry, D. T. Papageorgiou, and P. G. Petropoulos, "Electrohydrodynamic linear stability of two immiscible fluids in channel flow," Electrochimica Acta, vol. 51, pp. 5316-5323, 2006.

[57] S. J. Kim, Y.-A. Song, P. L. Skipper, and J. Han, "Electrohydrodynamic Generation and Delivery of Monodisperse Picoliter Droplets Using a Poly(dimethylsiloxane) Microchip," Analytical Chemistry, vol. 78, pp. 80118019, 2006/12/01 2006.

[58] J. Zeleny, "Instability of Electrified Liquid Surfaces," Physical Review, vol. 10, pp. 1-6, 1917.

[59] G. Taylor, "Disintegration of Water Drops in an Electric Field," Proceedings of the Royal Society of London. Series A. Mathematical and Physical Sciences, vol. 280, pp. 383-397, July 28, 19641964.

[60] A. Jaworek and A. T. Sobczyk, "Electrospraying route to nanotechnology: An overview," Journal of Electrostatics, vol. 66, pp. 197-219, 2008.

[61] O. Ozen, N. Aubry, D. T. Papageorgiou, and P. G. Petropoulos, "Monodisperse Drop Formation in Square Microchannels," Physical Review Letters, vol. 96, p. 144501, 2006.

[62] M. G. Lippmann, "Relations entre les phénomènes électriques et capillaires," 1875.

[63] A. Watanabe, M. Matsumoto, H. Tamai, and R. Gotoh, "Electrocapillary phenomena at oil-water interfaces," Kolloid-Zeitschrift und Zeitschrift für Polymere, vol. 220, pp. 152-159, 1967/10/01 1967.

[64] A. Watanabe, M. Matsumoto, R. Gotoh, and H. Tamai, "Electrocapillary phenomena at oil-water interfaces," Kolloid-Zeitschrift und Zeitschrift für Polymere, vol. 221, pp. 47-52, 1967/11/01 1967.

[65] A. Watanabe, M. Matsumoto, H. Tamai, and R. Gotoh, "Electrocapillary phenomena at oil-water interfaces," Kolloid-Zeitschrift und Zeitschrift für Polymere, vol. 228, pp. 58-63, 1968/11/01 1968.

[66] J. Berthier and K. A. Brakke, "Droplets: Shape, Surface and Volume," in The Physics of Microdroplets, ed: John Wiley \& Sons, Inc., 2012, pp. 83-103.

[67] D. C. Duffy, J. C. McDonald, O. J. A. Schueller, and G. M. Whitesides, "Rapid Prototyping of Microfluidic Systems in Poly(dimethylsiloxane)," Analytical Chemistry, vol. 70, pp. 4974-4984, 1998/12/01 1998. 
[68] J. C. McDonald and G. M. Whitesides, "Poly(dimethylsiloxane) as a Material for Fabricating Microfluidic Devices," Accounts of Chemical Research, vol. 35, pp. 491-499, 2002/07/01 2002.

[69] Q. Xu and M. Nakajima, "The generation of highly monodisperse droplets through the breakup of hydrodynamically focused microthread in a microfluidic device," Applied Physics Letters, vol. 85, pp. 3726-3728, 2004.

[70] S. Takeuchi, P. Garstecki, D. B. Weibel, and G. M. Whitesides, "An Axisymmetric Flow-Focusing Microfluidic Device," Advanced Materials, vol. 17, pp. 1067-1072, 2005.

[71] H. Shih-Hao, T. Wei-Heong, T. Fan-Gang, and T. Shoji, "A monolithically three-dimensional flow-focusing device for formation of single/double emulsions in closed/open microfluidic systems," Journal of Micromechanics and Microengineering, vol. 16, p. 2336, 2006.

[72] W. L. Ong, J. Hua, B. Zhang, T.Y. Teo, J. Zhuo, N.-T. Nguyen, N. Ranganathan, and L. Yobas, "Experimental and computational analysis of droplet formation in a high-performance flow-focusing geometry," Sensors and Actuators A: Physical, vol. 138, pp. 203-212, 2007.

[73] L. Yobas, S. Martens, W.L. Ong, and N. Ranganathan, "High-performance flow-focusing geometry for spontaneous generation of monodispersed droplets," Lab on a Chip, vol. 6, pp. 1073-1079, 2006.

[74] L. Rayleigh, "On the Capillary Phenomena of Jets," Proceedings of the Royal Society of London, vol. 29, pp. 71-97, January 1, 18791879.

[75] A. R. Abate, A. Poitzsch, Y. Hwang, J. Lee, J. Czerwinska, and D. A. Weitz, "Impact of inlet channel geometry on microfluidic drop formation," Physical Review E, vol. 80, p. 026310, 2009.

[76] Y.-C. Tan, V. Cristini, and A. P. Lee, "Monodispersed microfluidic droplet generation by shear focusing microfluidic device," Sensors and Actuators B: Chemical, vol. 114, pp. 350-356, 2006.

[77] M. C. Jerry, K. Ming-Che, and L. Chien-Po, "Control of Droplet Generation in Flow-Focusing Microfluidic Device with a Converging-Diverging NozzleShaped Section," Japanese Journal of Applied Physics, vol. 50, p. 107301, 2011.

[78] T. Cubaud and T. G. Mason, "Capillary threads and viscous droplets in square microchannels," Physics of Fluids (1994-present), vol. 20, pp. -, 2008.

[79] H. Liu and Y. Zhang, "Droplet formation in microfluidic cross-junctions," Physics of Fluids (1994-present), vol. 23, pp. -, 2011.

[80] J. Tan, J. H. Xu, S. W. Li, and G. S. Luo, "Drop dispenser in a cross-junction microfluidic device: Scaling and mechanism of break-up," Chemical Engineering Journal, vol. 136, pp. 306-311, 2008.

[81] L. Wu, M. Tsutahara, L. S. Kim, and M. Ha, "Three-dimensional lattice Boltzmann simulations of droplet formation in a cross-junction microchannel," International Journal of Multiphase Flow, vol. 34, pp. 852-864, 2008.

[82] C.-Y. Lee, Y.-H. Lin, and G.-B. Lee, "A droplet-based microfluidic system capable of droplet formation and manipulation," Microfluidics and Nanofluidics, vol. 6, pp. 599-610, 2009/05/01 2009.

[83] S. M. S. Murshed, S. H Tan, N.-T Nguyen, T. W Wong, and L. Yobas, "Microdroplet formation of water and nanofluids in heat-induced microfluidic Tjunction," Microfluidics and Nanofluidics, vol. 6, pp. 253-259, 2009/02/01 2009. 
[84] Y. Sun, Y. C. Kwok, and N. T. Nguyen, "A circular ferrofluid driven microchip for rapid polymerase chain reaction," Lab on a Chip, vol. 7, pp. 1012-1017, 2007.

[85] Y. Sun, Y.-C. Kwok, P. Foo-Peng Lee, and N.-T. Nguyen, "Rapid amplification of genetically modified organisms using a circular ferrofluid-driven PCR microchip," Analytical and Bioanalytical Chemistry, vol. 394, pp. 1505-1508, 2009/07/01 2009.

[86] A. C. Siegel, S. S. Shevkoplyas, D. B. Weibel, D. A. Bruzewicz, A. W. Martinez, and G. M. Whitesides, "Cofabrication of Electromagnets and Microfluidic Systems in Poly(dimethylsiloxane)," Angewandte Chemie International Edition, vol. 45, pp. 6877-6882, 2006.

[87] W. Lee, L. M. Walker, and S. L. Anna, "Role of geometry and fluid properties in droplet and thread formation processes in planar flow focusing," Physics of Fluids (1994-present), vol. 21, pp. -, 2009.

[88] J.-C. Baret, S. H. Tan, and B. Semin, "Method and fluidic microsystem for generating droplets dispersed in a continuous phase," ed: Google Patents, 2013.

[89] S. H. Tan, B. Semin, and J.-C. Baret, "Microfluidic flow-focusing in ac electric fields," Lab on a Chip, vol. 14, pp. 1099-1106, 2014.

[90] L. Chang-Soo, C. Chang-Hyung, and J. Jae-Hun, "Microfluidic system for the synthesis of functional materials," in Nano/Micro Engineered and Molecular Systems (NEMS), 2010 5th IEEE International Conference on, 2010, pp. 902905.

[91] J. H. Leamon, D. R. Link, M. Egholm, and J. M. Rothberg, "Overview: methods and applications for droplet compartmentalization of biology," Nat Meth, vol. 3, pp. 541-543, 2006.

[92] L. Mazutis, J. Gilbert, W. L. Ung, D. A. Weitz, A. D. Griffiths, and J. A. Heyman, "Single-cell analysis and sorting using droplet-based microfluidics," Nat. Protocols, vol. 8, pp. 870-891, 2013.

[93] J. N. Lee, C. Park, and G. M. Whitesides, "Solvent Compatibility of Poly(dimethylsiloxane)-Based Microfluidic Devices," Analytical Chemistry, vol. 75, pp. 6544-6554, 2003/12/01 2003.

[94] Z. Li, S. Y. Mak, A. Sauret, and H. C. Shum, "Syringe-pump-induced fluctuation in all-aqueous microfluidic system implications for flow rate accuracy," Lab on a Chip, vol. 14, pp. 744-749, 2014.

[95] Q. Brosseau, J. Vrignon, and J.-C. Baret, "Microfluidic Dynamic Interfacial Tensiometry ([small mu ]DIT)," Soft Matter, vol. 10, pp. 3066-3076, 2014.

[96] J.-C. Baret, "Surfactants in droplet-based microfluidics," Lab on a Chip, vol. 12, pp. 422-433, 2012.

[97] L. L. F. Agostinho, G. Tamminga, C. U. Yurteri, S. P. Brouwer, E. C. Fuchs, and J. C. M. Marijnissen, "Morphology of water electrosprays in the simple-jet mode," Physical Review E, vol. 86, p. 066317, 2012.

[98] S. H. Tan, F. Maes, B. Semin, J. Vrignon, and J.-C. Baret, "The Microfluidic Jukebox," Sci. Rep., vol. 4, 2014.

[99] M. Minnaert, "XVI. On musical air-bubbles and the sounds of running water," Philosophical Magazine Series 7, vol. 16, pp. 235-248, 1933/08/01 1933. 


\section{F Curriculum Vitae}

\section{Personal details}

Name: Say Hwa Tan

Date of birth: $4^{\text {th }}$ June 1982

Nationality: Singaporean

\section{Education}

2010-2014 Doctorate Research, Max Planck Institute for Dynamic and Selforganization, Goettingen, Germany.

2008-2010 Master of Engineering (Mech), Nanyang Technological University of Singapore.

2004-2008 Bachelor of Engineering (Mech), Nanyang Technological University of Singapore.

\section{Awards}

$1999 \quad$ Edusave scholarship for top academic performance.

2003 Police Coast Guard Commander's Award for resourcefulness and devotion to duty.

2004 Commissioner of Police Testimonial for contribution and leadership abilities.

2004 Police National Service Commendation for being top NSF officer in cohort.

2006 1st Runner-up in design-and-build rocket competition organized by NTU.

2010 Max Planck Institute research scholarship. 
Tan Kah Kee Postgraduate award for excellent academic achievements and commitment to serving the community presented by the 7th President of Singapore, Dr Tony Tan Keng Yam.

$2014 \quad$ Tan Kah Kee Young Inventor's Award 2014. Merit Prize.

\section{Publication Records}

\section{Book Chapters}

1. Say Hwa Tan, Nam Trung Nguyen: Active Control of Droplet Formation Process in Microfluidics. 01/2012: pages 51-75.

\section{Journal Publications}

1. Say Hwa Tan, Florine Maes, Benoît Semin, Jeremy Vrignon, and JeanChristophe Baret: The Microfluidic Jukebox. Sci. Rep., vol. 4, 2014.

2. Say Hwa Tan, Benoît Semin, Jean-Christophe Baret: Microfluidic flowfocusing in ac electric fields. Lab on a Chip 01/2014.

3. Say Hwa Tan, Nam-Trung Nguyen: Generation and manipulation of monodispersed ferrofluid emulsions: the effect of a uniform magnetic field in flow-focusing and T-junction configurations. Physical Review E 09/2011; 84(3 Pt 2):036317.

4. Nam-Trung Nguyen, Guiping Zhu, Yong-Chin Chua, Vinh-Nguyen Phan, Say Hwa Tan: Magnetowetting and sliding motion of a sessile ferrofluid droplet in the presence of a permanent magnet. Langmuir 08/2010; 26(15):12553-9.

5. Say Hwa Tan, Nam-Trung Nguyen, Levent Yobas, Tae Goo Kang: Formation and manipulation of ferrofluid droplets at a microfluidic T-junction. Journal of Micromechanics and Microengineering 03/2010; 20(4):045004.

6. Chaolong Song, Nam-Trung Nguyen, Anand Krishna Asundi, Say Hwa Tan: Tunable micro-optofluidic prism based on liquid-core liquid-cladding configuration. Optics Letters 02/2010; 35(3):327-9.

7. Chaolong Song, Nam-Trung Nguyen, Say Hwa Tan, Anand Krishna Asundi: A tuneable micro-optofluidic biconvex lens with mathematically predictable focal length. Microfluidics and Nanofluidics 01/2010; 9(4):889-896. 
8. Say Hwa Tan, Nam-Trung Nguyen, Yong Chin Chua, Tae Goo Kang: Oxygen plasma treatment for reducing hydrophobicity of a sealed polydimethylsiloxane microchannel. Biomicrofluidics 01/2010; 4(3):32204.

9. Chaolong Song, Nam-Trung Nguyen, Say Hwa Tan, Anand Krishna Asundi: Modelling and optimization of micro optofluidic lenses. Lab on a Chip 06/2009; 9(9):1178-84.

10.S. M. Sohel Murshed, Say Hwa Tan, Nam-Trung Nguyen, Teck Neng Wong, Levent Yobas: Microdroplet formation of water and nanofluids in heat-induced microfluidic T-junction. Microfluidics and Nanofluidics 01/2009; 6(2):253-259.

11. Yit-Fatt Yap, Say Hwa Tan, Nam-Trung Nguyen, S M Sohel Murshed, TeckNeng Wong, Levent Yobas: Thermally mediated control of liquid microdroplets at a bifurcation. Journal of Physics D Applied Physics 01/2009; 42.

12. Chaolong Song, Nam-Trung Nguyen, Say Hwa Tan, Anand Krishna Asundi: A micro optofluidic lens with short focal length. Journal of Micromechanics and Microengineering 01/2009; 19:85012-6.

13.S. M. Sohel Murshed, Say Hwa Tan, Nam-Trung Nguyen: Temperature dependence of interfacial properties and viscosity of nanofluids for dropletbased microfluidics. Journal of Physics D Applied Physics 01/2008; 41:0-5.

14. Say Hwa Tan, S. M. Sohel Murshed, Nam-Trung Nguyen, Teck Neng Wong, Levent Yobas: Thermally controlled droplet formation in flow focusing geometry: formation regimes and effect of nanoparticle suspension. Journal of Physics D Applied Physics 01/2008; 41:165501-7.

15. Nam-Trung Nguyen, Teck Hui Ting, Yit-Fatt Yap, Teck Neng Wong, John Chee Kiong Chai, Wee Liat Ong, Junlong Zhou, Say Hwa Tan, Levent Yobas: Thermally mediated droplet formation in microchannels. Applied Physics Letters 09/2007;

16. Jing Liu, Say Hwa Tan, Yit Fatt Yap, Min Yuan Ng, Nam-Trung Nguyen: Numerical and experimental investigations of the formation process of ferrofluid droplets. Microfluidics and Nanofluidics 11(2):177-187. 


\section{Patents}

Application number: PCT/EP2012/001423

Publication date: Oct 3, 2013

Filing date: $\quad$ Mar 30, 2012

Inventors: $\quad$ Jean-Christophe Baret, Say Hwa Tan, Benoit Semin.

Title: $\quad$ Method and fluidic microsystem for generating droplets dispersed in a continuous phase

Link: http://patentscope.wipo.int/search/en/WO2013143562

\section{Conference Proceedings}

1. S. H. Tan, B. Semin, and J.-C. Baret, "Novel Electrical Control in Droplet Microfluidics Using an AC Electric Field," Proceedings of ASME 2013.

2. S. H. Tan, B. Semin, F. Maes, and J.-C. Baret, "Electric control in dropletbased microfluidic," Proceedings of MicroTas 2012.

3. N.-T Nguyen, S. M. S. Murshed, and S. H Tan, "Investigation of TemperatureDependent Droplet Formation of Nanofluids in Microfluidic T-Junction," 01/2008: pages 517-521; ASME, ISBN: 0-7918-4294-0

\section{Professional Services}

Reviewer for the following journals

1. Microfluidics and Nanofluidics - Springer

2. Biomedical Microdevices - Springer

3. Micro and Nanosystems 